\title{
Digitale Medienbildung im schulischen Arabischunterricht
}

\author{
Masterarbeit \\ im Masterstudiengang \\ Arabistik \\ in der Fakultät Geistes- und \\ Kulturwissenschaften \\ der Otto-Friedrich-Universität \\ Bamberg
}

Verfasserin: Paula Rötscher

Prüfer: Dr. Peter Konerding

Zweitprüferin: Prof. Dr. Lale Behzadi 
URN: urn:nbn:de:bvb:473-irb-495035

DOI: https://doi.org/10.20378/irb-49503

Dieses Werk ist als freie Onlineversion über das Forschungsinformationssystem (FIS; https://fis.uni-bamberg.de) der Universität Bamberg erreichbar. Das Werk steht unter der CC-Lizenz CC-BY.

(c) 


\section{Inhaltsverzeichnis}

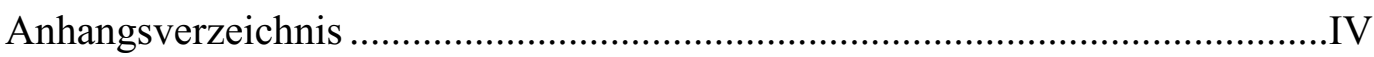

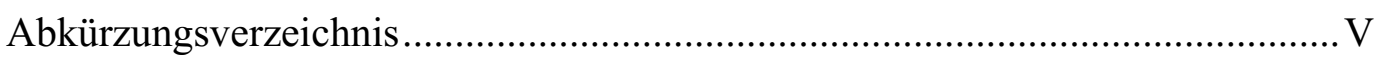

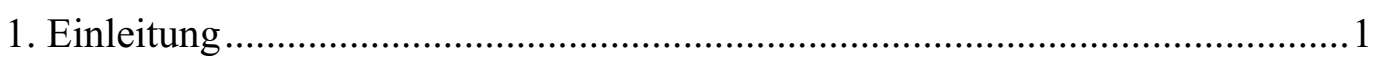

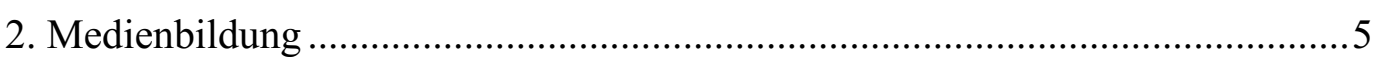

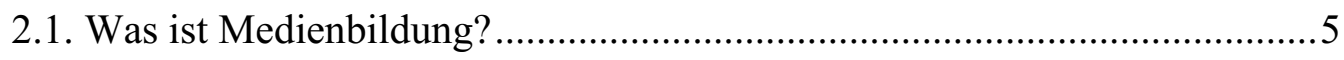

2.1.1. Begriffserklärung Medienbildung und Medienkompetenz....................5

2.1.2. Medienbildung folgt dem Primat des Pädagogischen ...........................6

2.1.3. Was sind neue Medien? .................................................................... 6

2.2. Medienbildung und schulischer Arabischunterricht .................................

2.2.1. Medienbildung als Pflichtauftrag schulischer Bildung .......................7

2.2.2. Medienbildung im Fremdsprachenunterricht ..................................... 8

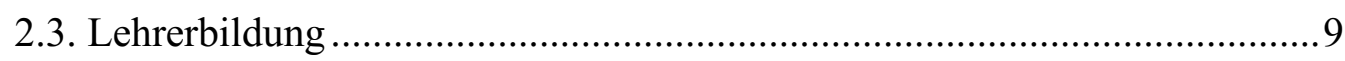

2.3.1. Die Rolle der Lehrkraft in der Förderung von Medienbildung .............9

2.3.2. Fort- und Weiterbildungen im Bereich Medienbildung ..................... 10

2.3.3. Vernetzung von Arabischlehrer*innen ............................................ 12

2.3.4. Institutionelle Verankerung der Arabischdidaktik ............................. 13

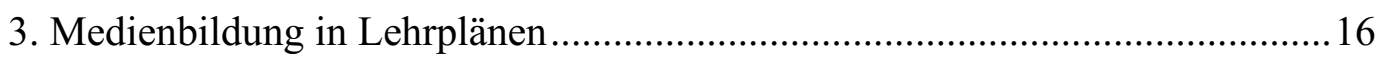

3.1. Integration medienpädagogischer Ziele im Lehrplan............................... 16

3.2. Medienpädagogische Defizite in Deutschland ........................................ 17

3.3. Analyse von Lehrplänen für fremdsprachlichen Arabischunterricht ......... 18

3.3.1. Lehrplan der Salzmannschule Schnepfenthal .................................... 18

3.3.2. Lehrplan für das Lycée in Frankreich ..............................................2 21

3.3.3. Lehrplan der Edmonton Public Schools in Alberta, Kanada ............... 24

3.3.4. Lehrplan der Fairfax County Public Schools in Virginia, USA..........27

3.3.5. Lehrplan für New South Wales, Australien......................................... 31

3.4. Erkenntnisse in Bezug auf die Vorgaben der KMK................................. 35

4. Anwendungsbeispiele für digitale Medien im Arabischunterricht ....................38

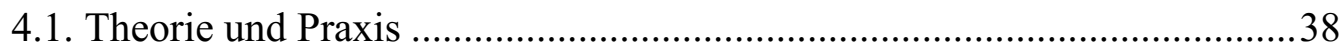

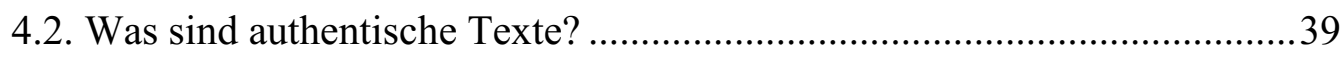

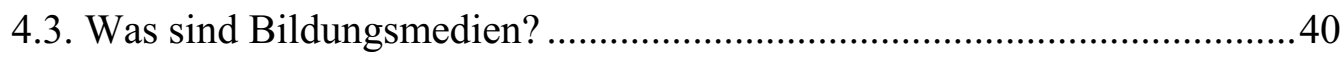

4.4. Neue Medien und die Varietäten der arabischen Sprache......................... 41

4.4.1. Erstellung passgenauer Bildungsmedien ...........................................4 41 
4.4.2 Arabischsprachige Fernsehserien .42

4.4.3. Ausbildung von Sprachbewusstheit 43

4.4.4. Varietäten des geschriebenen Arabisch 44

4.4.5. Unterschiede in der Aussprache .45

4.4.6. Verstärkung der Authentizität sprachlicher Situationen .46

4.5. Kommunikation, Vernetzung und Austausch .46

4.5.1. Sofortnachrichtendienste .46

4.5.2. Vernetzung von Lernenden - communities of practice .47

4.5.3. Schreiben mit E-Mail und Textverarbeitungsprogrammen .48

4.5.4. Vermittlung zwischen „West“" und „Ost“" 49

4.6. Angeleitete und eigenständige Recherche ................................................50

4.6.1. Recherche mit geringen Arabischkenntnissen .................................50

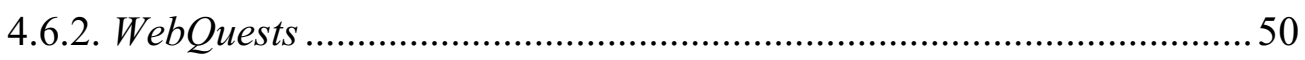

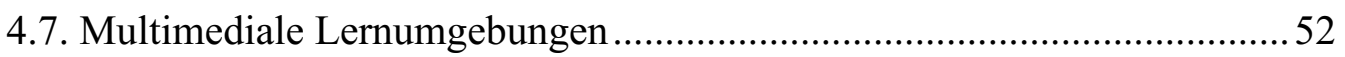

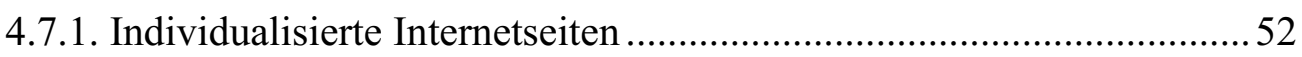

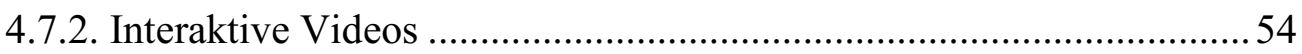

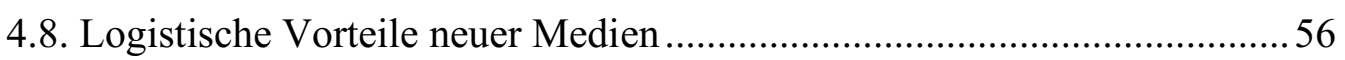

4.9. Erkenntnisse für den deutschen schulischen Kontext ...............................57

5. Vergleich existenter (Bildungs-)medien ......................................................5

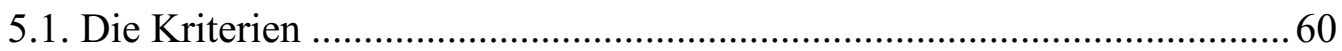

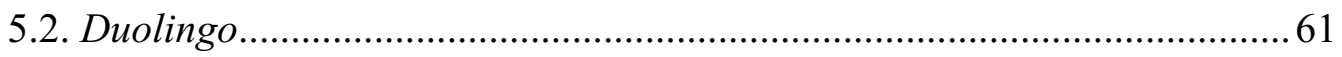

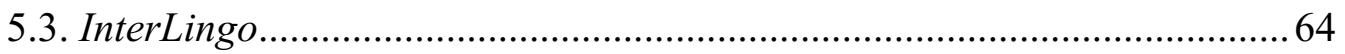

5.4. Defense Language Institute Foreign Language Center eLearning ...........66 66

5.4.1. Arabic Phone Conversations ................................................................67

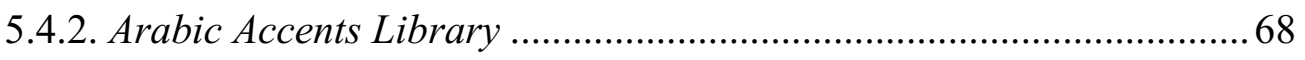

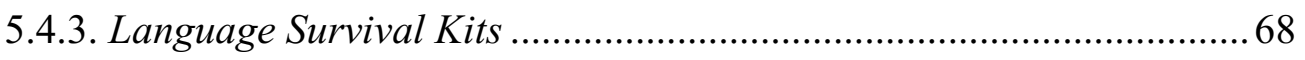

5.4.4. Global Language Online Support System (GLOSS).......................... 69

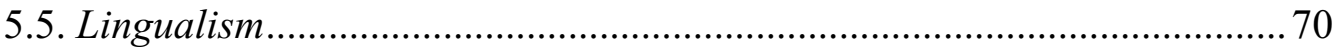

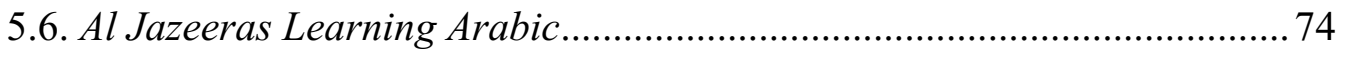

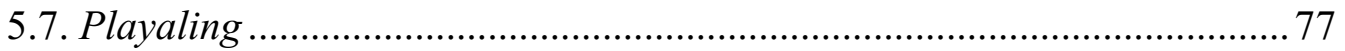

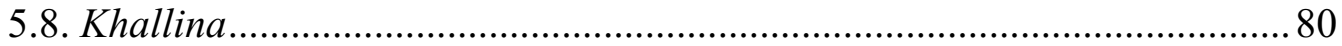

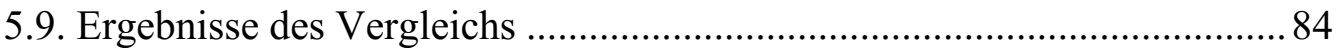

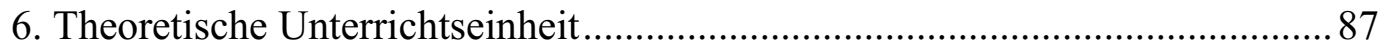

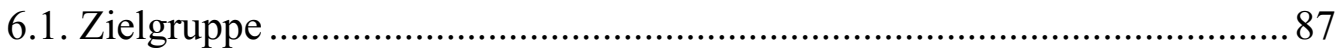

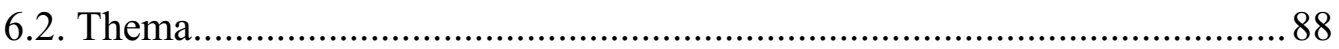




6.3. Aufbau




\section{Anhangsverzeichnis}

Anhang I. Transkript für die Präsentationsphase der theoretischen Unterrichtseinheit

Anhang II. Vokabelliste für die Präsentationsphase der theoretischen

Unterrichtseinheit .ii

Anhang III. Lückensätze für die Einübungsphase der theoretischen

Unterrichtseinheit iii

Anhang IV. Wortgruppen für die Einübungsphase der theoretischen Unterrichtseinheit iv

Anhang V. Vokabelkarten für die Transferphase der theoretischen Unterrichtseinheit $\mathrm{V}$

Anhang VI. Lückentext für die Transferphase der theoretischen

Unterrichtseinheit vi 


\section{Abkürzungsverzeichnis}

$\begin{array}{ll}\text { AraF } & \text { Arabisch als Fremdsprache } \\ \text { DLI } & \text { Defense Language Institute } \\ \text { DLIFLC } & \text { Defense Language Institute Foreign Language Center } \\ \text { ESA } & \text { Educated Spoken Arabic } \\ \text { GLOSS } & \text { Global Language Online Support System } \\ \text { KMK } & \text { Kultusministerkonferenz } \\ \text { OER } & \text { Open Educational Resources } \\ \text { vzbv } & \text { Verbraucherzentrale Bundesverband }\end{array}$

In der vorliegenden Forschungsarbeit wird die wissenschaftliche Umschrift der arabischen Sprache für die orientalistischen Fächer der Universität Bamberg verwendet. $^{1}$

${ }^{1}$ Vgl. Hildebrandt. 



\section{Einleitung}

Im deutschsprachigen Raum ist in den letzten Jahren ein gestiegenes Interesse an den arabischen Ländern und ihren Kulturen zu verzeichnen. ${ }^{2}$ Dies begründet sich unter anderem in den wirtschaftlichen, politischen und militärischen Beziehungen zwischen Deutschland und den aufstrebenden Golfstaaten sowie in der Entwicklung militärischer Auseinandersetzungen in verschiedenen arabischen Ländern, die sich durch daraus resultierende Migrationsbewegungen auch auf die deutsche Gesellschaft auswirken. ${ }^{3}$ Arabisch ist eine der sechs offiziellen Sprachen der Vereinten Nationen ${ }^{4}$, wird schätzungsweise von 290 Millionen Menschen als Muttersprache gesprochen ${ }^{5}$ und ist in 27 Ländern der Welt Amts- und Gerichtssprache. ${ }^{6}$ Als Schulfach ist Arabisch in Deutschland jedoch kaum vorzufinden und wenn, dann vorrangig in Form von herkunftssprachlichem Unterricht oder als Wahlfach. ${ }^{7}$ Demzufolge ergibt sich eine Diskrepanz zwischen der Bedeutung der arabischen Sprache und den Möglichkeiten für deutsche Schüler*innen, sie als Fremdsprache zu erlernen. Um diese Diskrepanz zu beheben, sollte danach gestrebt werden, Arabisch als fremdsprachliches Schulfach auszuweiten und zu formalisieren. Hierzu muss unter anderem ein Lehrplan konzipiert werden, der den Vorgaben der Kultusministerkonferenz (KMK) und des jeweiligen Bundeslandes entspricht. Eine grundlegende Anforderung der KMK ist es, Medienbildung in allen Fachlehrplänen zu integrieren und die Ausbildung digitaler Kompetenzen als eines der Unterrichtsziele anzuerkennen. ${ }^{8}$

Folglich versucht diese Forschungsarbeit zu beantworten, ob und wie Medienbildung bereits in den Arabischunterricht integriert wird und in welcher Weise sie gefördert werden kann. Die Forschungsarbeit beginnt mit einer Erläuterung der Medienbildung an sich und ihrer Bedeutung für den schulischen Fremdsprachenunterricht. Daraufhin folgt eine Analyse verschiedener Arabischlehrpläne in Bezug auf die Beachtung von Medienbildung und die Ausbildung konkreter Medienkom-

\footnotetext{
${ }^{2}$ Vgl. Behzadi und Konerding 2018, S. 235.

${ }^{3}$ Vgl. Behzadi et al. 2019, S. 406.

${ }^{4}$ Vgl. United Nations.

${ }^{5}$ Vgl. Ehlion Team 2019.

${ }^{6}$ Vgl. Bundesverband der Dolmetscher und Übersetzer 2017, S. 9.

${ }^{7}$ Vgl. Behzadi et al. 2019, S. 411.

${ }^{8}$ Vgl. Sekretariat der Kultusministerkonferenz 2016, S. 25.
} 
petenzen. An die Erkenntnisse aus den Lehrplänen schließt eine Auswahl von Anwendungsbeispielen für den sinnstiftenden Einsatz neuer Medien im Arabischunterricht an. Daraufhin werden bereits existente digitale Medien für den Arabischunterricht anhand von Kriterien der KMK und des Verbraucherzentrale Bundesverbands (vzbv) bewertet. Abschließend präsentiert diese Forschungsarbeit einen theoretischen Vorschlag für eine Unterrichtseinheit, welche Aspekte der Medienbildung und Ansätze für die Ausbildung von Medienkompetenz umsetzt und mit konkreten sprachlichen Zielsetzungen verbindet.

Zu Beginn wird der Unterschied zwischen Medienbildung und Medienkompetenz erläutert und verdeutlicht, warum beide Konzepte wesentlich für den Unterricht an deutschen Schulen sind. Es wird hervorgehoben, warum Medienbildung auch im Fremdsprachenunterricht umgesetzt werden muss und welche Rolle die Lehrkraft in diesem Zusammenhang spielt. An dieser Stelle wird auf das Nichtvorhandensein einer formalisierten Ausbildung für Arabischlehrer*innen in Deutschland und die sich daraus ergebenden Defizite im Bereich Medienpädagogik eingegangen. Es werden unter anderem die Wichtigkeit der Vernetzung von Arabischlehrkräften und die Notwendigkeit digitaler Fortbildungsangebote hervorgehoben. In diesem Teil dienen vorrangig die Anforderungen und Vorgaben der KMK sowie Fachliteratur zur Medienbildung als Basis der Forschung.

Darauf folgt eine Analyse verschiedener Arabischlehrpläne in Bezug auf die Verankerung medienpädagogischer Prinzipien. Aktuell gibt es in Deutschland nur zwei Arabischlehrpläne für den Arabischunterricht an der Salzmannschule Schnepfenthal in Thüringen. Diese Lehrpläne stammen aus den Jahren 2002 und 2007 und können als Ausgangsbasis für einen neuen Lehrplan dienen. Sie bedürfen jedoch einer Aktualisierung, um auf die neuen Anforderungen, die der digitale Wandel mit sich bringt, zu reagieren. Laut KMK sollten existente Lehr- und Bildungspläne immer wieder überarbeitet und dabei gegebenenfalls auch neue bzw. präzisierte Anforderungen formuliert werden. ${ }^{9}$ Um solche präzisierten Anforderungen zu entwickeln, werden die Thüringer Lehrpläne, als Beispiel für fremdsprachlichen Arabischunterricht in Deutschland, sowie weitere Arabischlehrpläne aus Frankreich, Kanada, den USA und Australien miteinander vergli-

\footnotetext{
${ }^{9}$ Vgl. Sekretariat der Kultusministerkonferenz 2016, S. 11.
} 
chen. Ziel soll es sein, konkrete Beispiele für die Umsetzung von Medienbildung und die Förderung von Medienkompetenz zu finden und zu untersuchen, inwieweit diese für den Arabischunterricht im deutschsprachigen Raum übernommen werden könnten. Hierbei orientiert sich die Analyse vorrangig an den von der KMK zusammengestellten Kompetenzen in der digitalen Welt $^{10}$, welche dazu dienen sollen, die Überprüfung und Überarbeitung bestehender Lehrpläne hinsichtlich ihres Beitrags zur Förderung von Medienkompetenz zu standardisieren und zu vereinfachen. ${ }^{11}$

Um ein ganzheitliches Bild von der Verwirklichung medienpädagogischer Ansätze im Arabischunterricht $\mathrm{zu}$ präsentieren, werden im darauffolgenden Teil Anwendungsbeispiele für den Einsatz neuer Medien und Erfahrungsberichte aus dem Ausland vorgestellt. Da der Arabischunterricht im deutschsprachigen Raum bisher wenig verbreitet ist, stammen die verwendeten Beispiele vorrangig von amerikanischen Universitäten. Der Vergleich dieser Beispiele soll zeigen, welche Unterrichtsziele und sprachlichen Kompetenzen durch den Einsatz verschiedener Medien unterstützt werden können. Auch hier wird immer wieder Bezug auf die Kompetenzen in der digitalen Welt und anderweitige von der KMK formulierte Vorgaben genommen. Diese Erkenntnisse sollen in Kombination mit den Erkenntnissen der Lehrplananalyse dazu dienen, potenzielle Anwendungsbereiche für den sinnvollen, gezielten Einsatz neuer Medien im Arabischunterricht zu skizzieren.

Im Anschluss an die Analyse der Arabischlehrpläne und den Vergleich verschiedener Anwendungsbeispiele folgt eine Bewertung bereits existenter digitaler Medien für den Arabischunterricht. Als Bewertungsbasis dienen einerseits die Kriterien der KMK für sinnvolle Bildungsmedien, welche unter anderem die Interaktivität, Veränderbarkeit und Teilbarkeit digitaler Medien umfassen. ${ }^{12}$ Andererseits finden die Kriterien des vzbv Beachtung, welche sich mit der Gestaltung und dem Aufbau des Mediums, den vermittelten Inhalten und der methodischen Vielfalt beschäftigen. ${ }^{13}$ Die Stärken und Schwächen eines jeden Mediums werden herausgearbeitet, um zu verdeutlichen, inwiefern digitale Medien den Arabischunterricht konkret unterstützen können.

\footnotetext{
${ }^{10}$ Vgl. Ständige Konferenz der Kultusminister der Länder in der Bundesrepublik Deutschland 2016.

${ }^{11}$ Vgl. Sekretariat der Kultusministerkonferenz 2016, S. 19.

${ }^{12}$ Vgl. ebd., S. 33.

${ }^{13}$ Vgl. Verbraucherzentrale Bundesverband.
} 
Der letzte Teil dieser Forschungsarbeit greift die bis dahin herausgearbeiteten Probleme auf und präsentiert eine theoretische Unterrichtseinheit für fremdsprachlichen Arabischunterricht, die sowohl bestimmte sprachliche Ziele als auch Prinzipien der Medienbildung verwirklicht. Die Konzipierung dieser Unterrichtseinheit basiert auf den Ansätzen der kritisch-konstruktiven Didaktik nach Klafki ${ }^{14}$ und dem Lehrphasenmodell von Zimmermann. ${ }^{15}$ Die Auswahl des Themas „Soziale Medien“ für die Unterrichtseinheit wird ausführlich begründet und jede Phase des Unterrichts im Detail beschrieben. Des Weiteren werden Vorschläge für mögliche Anpassungen und anderweitige Unterrichtsaktivitäten gemacht und es wird erläutert, inwiefern diese Unterrichtseinheit die Vermittlung medienpädagogischen Wissens, konkreter Medienkompetenzen und neuer sprachlicher Mittel miteinander verbindet und umsetzt.

Diese Forschungsarbeit ist einerseits von Bedeutung, weil sie Medienbildung an sich näher erforscht, welche laut KMK zum Bildungsauftrag der Schule gehört und als Kulturtechnik mittlerweile genauso wichtig ist wie Lesen, Rechnen und Schreiben. ${ }^{16}$ Andererseits wird in dieser Arbeit jedoch auch ein erster Versuch unternommen, die Ziele der schulischen Medienbildung mit den angestrebten Zielen und Kompetenzen des fremdsprachlichen Arabischunterrichts zu verbinden. Dies soll nicht zuletzt dazu dienen, eine Basis zu schaffen, um auf die zukünftige Formalisierung des Arabischunterrichts an deutschen Schulen hinzuarbeiten. Dieses Ziel würde die Entwicklung formeller Arabischlehrpläne erfordern, welche unter anderem die Vorgaben der KMK für Medienbildung beinhalten müssen. Folglich ist für die Etablierung des Arabischen als Fremdsprache an deutschen Schulen die Umsetzung medienpädagogischer Ziele nicht optional, sondern unumgänglich. Diese Forschungsarbeit versucht $\mathrm{zu}$ zeigen, in welchem Umfang medienpädagogische Ansätze bereits im Arabischunterricht umgesetzt werden, wo aktuelle Defizite liegen und welche möglichen Handlungsvorschläge und Anwendungsbereiche zur Förderung von Medienbildung im Arabischunterricht beitragen können.

\footnotetext{
${ }^{14}$ Vgl. Klafki 2007, S. 272.

${ }^{15}$ Vgl. Zimmermann 1988, zitiert nach Nieweler 2017, S. 194-196.

${ }^{16}$ Vgl. Ständige Konferenz der Kultusminister der Länder in der Bundesrepublik Deutschland 2012, S. 9.
} 


\section{Medienbildung}

\subsection{Was ist Medienbildung?}

\subsubsection{Begriffserklärung Medienbildung und Medienkompetenz}

Laut Spanhel führen die Begriffe Medienkompetenz und Medienbildung zwangsläufig zu ganz unterschiedlichen Zugangsweisen, Beschreibungen und Abgrenzungen im Oberbereich der Medienpädagogik. Während Medienkompetenz auf die Ausbildung bestimmter Fähigkeiten abzielt, deutet der Begriff Medienbildung auf den pädagogischen Ansatz der Mediennutzung hin. Die Medienbildung ist eine pädagogische Aufgabe, in der es darum geht, „Lernräume als mediale Bildungsräume zu gestalten“ und Schüler*innen im Umgang mit Medien auf intellektueller und moralischer Ebene zu begleiten. ${ }^{17}$ Auch die KMK unterscheidet deutlich zwischen diesen beiden Begriffen, welche längere Zeit lang synonym verwendet wurden: Medien dienen nicht nur dazu, Bildungsprozesse und Lerntransfers in methodisch-didaktischem Sinne zu fördern, sondern werden auch selbst zum Bildungsgegenstand. Folglich umfasst die schulische Medienbildung stets das Lernen mit Medien und das Lernen über Medien. Medienbildung ist das pädagogische Auseinandersetzen mit Medien und ein konkreter Bildungsauftrag, der sich an die Lehrkraft wendet und von ihr umgesetzt wird. Sie stellt den Schüler*innen verschiedene Arten von Medien vor, bezieht sie zielgerichtet in das Unterrichtsgeschehen ein, hält die Schüler*innen dazu an, das verfügbare Medienangebot kritisch zu reflektieren und veranschaulicht, wie aus diesem Angebot sinnvoll und bedürfnisbezogen gewählt werden kann, um verschiedene Ziele zu erreichen. ${ }^{18}$ Medienbildung ist der Prozess, durch den die sogenannte Medienkompetenz ausgebildet wird - die Kenntnisse und Fähigkeiten, die für ein sachgerechtes, selbstbestimmtes, kreatives und sozial verantwortliches Handeln in der medial geprägten Welt vonnöten sind. ${ }^{19}$ Es ist demnach wichtig, zwischen den beiden Begriffen zu unterscheiden und sie nicht als gleichbedeutend zu verwenden. Medienbildung ist nötig, um umfassende Medienkompetenzen auszubilden.

\footnotetext{
${ }^{17}$ Spanhel 2011, S. 97.

${ }^{18}$ Vgl. Ständige Konferenz der Kultusminister der Länder in der Bundesrepublik Deutschland 2012, S. 4.

${ }^{19}$ Vgl. ebd., S. 3.
} 


\subsubsection{Medienbildung folgt dem Primat des Pädagogischen}

Die KMK hebt zudem hervor, dass Medienbildung im schulischen Bereich dem Primat des Pädagogischen folgen muss. Oder anders ausgedrückt: Der Bildungsund Erziehungsauftrag hat Vorrang vor der Nutzung von Medien. Medien werden nicht einfach zum Selbstzweck in das Unterrichtsgeschehen mit eingebunden, sondern dienen als Hilfsmittel, um die anderweitig definierten Ziele des schulischen Bildungsauftrags zu verwirklichen. ${ }^{20}$ Dieser Punkt ist von großer Wichtigkeit für die allgemeine Diskussion zum Thema Medienbildung, da er dem verbreiteten Phänomen des Medieneinsatzes ohne konkrete pädagogische Zielsetzung entgegenwirkt. Beispielsweise ist laut einem Bericht der FriedrichEbert-Stiftung zu beobachten, dass in vielen deutschen Schulen technologische Neuerungen ermutigt und vorangetrieben werden, ohne dass dahinter ein fundiertes pädagogisches Konzept steckt. Dies führt dazu, dass technologischer Nutzen und etwaige Vorteile für den Unterricht nur selten $\mathrm{zu}$ langfristigen Veränderungen führen. ${ }^{21}$ Dieses Problem ist auch deutlich innerhalb der Arabischlehrpläne zu beobachten, die im Folgenden analysiert und verglichen werden. In vielen Lehrplänen ist die Verwendung neuer Medien zwar verankert, aber die zugrunde liegenden pädagogischen Ansätze werden selten erwähnt oder gar erklärt.

\subsubsection{Was sind neue Medien?}

Das Landesmedienzentrum Baden-Württemberg stellt neue Medien mit digitalen Medien gleich und definiert diese dementsprechend als ,alle Geräte, die zur Aufzeichnung, Berechnung, Verarbeitung und Verteilung von digitalen Inhalten gebraucht werden.“22 Eine andere verwendete Bezeichnung für neue Medien ist „elektronische Medien“. ${ }^{23}$ Bei Diskussionen über den Begriff „,neue Medien“ sollte man bedenken, dass dieser immer kontextbezogen ist. In den 1920er-Jahren galten Radios als neue Medien, zu Beginn des 21. Jahrhunderts Laptops. ${ }^{24}$ Versucht man,

\footnotetext{
${ }^{20}$ Vgl. Sekretariat der Kultusministerkonferenz 2016, S. 29.

${ }^{21}$ Vgl. Friedrich-Ebert-Stiftung 2017, S. 25.

${ }^{22}$ Landesmedienzentrum Baden-Württemberg 2019.

${ }^{23} \mathrm{Vgl}$. ebd.

${ }^{24}$ Vgl. Institut für Pädagogik der Elementar- und Primarstufe 2011.
} 
den Bereich der neuen Medien in unserer Zeit einzugrenzen, sind die ersten Assoziationen mobile Endgeräte wie Smartphones oder Tablets. Deshalb empfiehlt es sich, den Begriff digitale Medien zu verwenden, um zu verdeutlichen, dass unabhängig vom Erfindungsjahr oder von seither entstanden Neuerungen alle Geräte gemeint sind, die digitale Inhalte verarbeiten und verfügbar machen. Im Kontext dieser Masterarbeit werden die Begriffe neue Medien und digitale Medien synonym verwendet. Der Fokus liegt vorrangig auf Computern und dem Internet sowie den möglichen Software-, Kommunikations- und Textverarbeitungsprogrammen, Internetseiten und audiovisuellen Inhalten, die durch diese beiden digitalen Medien bereitgestellt werden können.

\subsection{Medienbildung und schulischer Arabischunterricht}

\subsubsection{Medienbildung als Pflichtauftrag schulischer Bildung}

Die KMK erklärt in ihrem Beschluss Medienbildung in der Schule von 2012, dass der Umgang mit digitalen Medien als Kulturtechnik den gleichen Stellenwert wie Lesen, Schreiben und Rechnen hat. ${ }^{25}$ Folglich muss Medienbildung als Pflichtaufgabe schulischer Bildung verankert und in alle Fächer einbezogen werden. ${ }^{26}$ Die KMK hebt weiterhin hervor, dass die Medienbildung kein separates Unterrichtsfach ist und keine fachdidaktische Tradition hat, weshalb jedes Unterrichtsfach seinen Beitrag leisten sollte, um sie zu fördern. ${ }^{27}$ Wenn von allen Schulfächern gesprochen wird, ist unweigerlich auch von den Fremdsprachen die Rede. Es gibt also keinen stichhaltigen Grund, warum sich die Arabischdidaktik aus dieser Diskussion heraushalten und sich nicht aktiv daran beteiligen sollte, die Ausbildung von Medienkompetenz unter Lernenden im Rahmen ihrer Möglichkeiten zu unterstützen. Auf den ersten Blick scheint diese Forderung vielleicht unrealistisch zu sein, da andere Fächer aus dem sozialwissenschaftlichen, politischen oder informationstechnischen Bereich einen direkteren Bezug zur Medienbildung und zur Verwendung von Medien haben. Aber die KMK betont diesbezüglich, dass nicht jedes Fach gleichermaßen zur Entwicklung aller angestrebten

\footnotetext{
${ }^{25}$ Vgl. Ständige Konferenz der Kultusminister der Länder in der Bundesrepublik Deutschland 2012, S. 9.

${ }^{26}$ Vgl. ebd., S. 3.

${ }^{27}$ Vgl. ebd., S. 6.
} 
medialen Kompetenzen beitragen muss. Vielmehr soll jedes Fach mit seinen spezifischen Zugängen zur digitalen Welt seinen Beitrag für die Entwicklung der formulierten Anforderungen leisten. ${ }^{28}$ Aufgabe der Arabischlehrkräfte in Deutschland ist es also, die Erwartungen und Vorgaben zu verstehen, als wichtig anzuerkennen und im Rahmen ihrer Möglichkeiten umzusetzen. Natürlich sind der Ausbildung der einzelnen Kompetenzen hier keine Grenzen gesetzt und es sollte auf Basis der schulischen Besonderheiten, der Erfahrungen und Fähigkeiten der Lehrkraft sowie den Zielen und Interessen der Schüler*innen möglich sein, individuelle Handlungsansätze zu definieren.

\subsubsection{Medienbildung im Fremdsprachenunterricht}

Die KMK betont in ihren Empfehlungen zudem, dass Technologie im Fremdsprachenunterricht eine nicht zu unterschätzende Rolle spielt, denn „Fremdsprachenunterricht nutzt und reflektiert zeitgemäße Medien“. ${ }^{29}$ Der Fremdsprachenunterricht an sich kann und sollte sich also aus der Förderung von Medienbildung nicht zurückziehen, denn auch hier müssen neue Medien nicht nur eingesetzt, sondern auch reflektiert werden. Hinter dieser Zielsetzung steckt wieder die Forderung, das Lernen über Medien zu fördern. Es reicht nicht aus, Videos, Filme oder Apps als Teil von Aufgabenstellungen oder Aktivitäten in den Fremdsprachenunterricht zu integrieren. Stattdessen muss auch dem Reflektieren und dem kritischen Umgang mit Medien, welche durch die Lehrkraft angeleitet werden, Raum gegeben werden.

Außerdem ist $\mathrm{zu}$ betonen, dass Medienbildung auch darauf abzielt, Kinder und Jugendliche auszubilden, welche eigene Maßstäbe und Kriterien für die Bewertung und den Gebrauch neuer Medien entwickeln. Sie sollen durch ihre schulische Bildung dazu befähigt werden, potenzielle Gefährdungen durch beispielsweise nicht altersgerechte oder strafrechtlich relevante Medienangebote zu erkennen und angebracht auf sie zu reagieren. ${ }^{30}$ Schüler*innen werden unweigerlich in der Schule und außerhalb mit verschiedensten medialen Inhalten in Berührung

\footnotetext{
${ }^{28}$ Vgl. Sekretariat der Kultusministerkonferenz 2016, S. 15.

${ }^{29}$ Ständige Konferenz der Kultusminister der Länder in der Bundesrepublik Deutschland 2011, S. 3.

${ }^{30}$ Vgl. Ständige Konferenz der Kultusminister der Länder in der Bundesrepublik Deutschland 2012, S. 6.
} 
kommen. Zudem zeigt sich in der folgenden Analyse verschiedener Arabischlehrpläne, dass Schüler*innen bewusst dazu angehalten werden, eigenständig arabische mediale Inhalte $\mathrm{zu}$ finden und für ihre Zwecke zu nutzen, beispielsweise zur Unterhaltung, oder um ihre sprachlichen Mittel zu erweitern. Vor allem bei fremdsprachigen Medien ist anzunehmen, dass die Schüler*innen einer größeren Gefahr unangebrachter Inhalte ausgesetzt sein könnten, da sie nicht immer alles Gesagte oder Gezeigte verstehen und einordnen können. Beispielsweise mangelt es ihnen an soziokulturellen, politischen oder religiösen Kenntnissen, um bestimmte Aussagen zu verstehen und als problematisch zu erkennen. Hier fällt der Arabischlehrkraft eine nicht zu unterschätzende Verantwortung zu, die Schüler*innen anzuleiten und ihnen zu zeigen, wie und wo sie vertrauenswürdige und altersgerechte arabischsprachige Quellen finden können. Diese Rolle kann und sollte von niemand anderem als der Arabischlehrkraft übernommen werden, denn sie kennt sowohl die Schüler*innen als auch die arabischsprachige mediale Landschaft und kann sie den Schüler*innen auf reflektierte und sichere Art und Weise näherbringen. Weder die Eltern noch die Mitschüler*innen oder Kolleg*innen sind in gleichem Maße qualifiziert, diese Aufgabe zu übernehmen.

\subsection{Lehrerbildung}

\subsubsection{Die Rolle der Lehrkraft in der Förderung von Medienbildung}

Wie zuvor erwähnt sollte der Nutzung von Medien im Unterricht ein klares, zielgerichtetes pädagogisches Konzept zugrunde liegen, welches sowohl das Lernen mit Medien als auch das Lernen über Medien beinhaltet. Vor allem der Lehrkraft fällt in der Umsetzung dieser Anforderungen eine ausschlaggebende Rolle zu. Sie soll laut Empfehlungen der KMK fähig sein, die Medienerfahrungen ihrer Schüler*innen $\mathrm{zu}$ verstehen und $\mathrm{zu}$ thematisieren, sie in der Analyse und Bewertung des bestehenden Medienangebotes anzuleiten, positive und negative Medienwirkungen zu veranschaulichen und zudem ihre Schüler*innen lehren, neue Medien für kreative Prozesse zu nutzen. Eine solche Vielfalt an Fähigkeiten kann nur vermittelt werden, wenn die Lehrkräfte selbst fundiertes medienpädagogisches Wissen und konkrete Medienkompetenzen besitzen. Hierbei betont die KMK, dass es nicht nur darum geht, dass Lehrer*innen im grundsätzlichen 
Umgang mit neuen Technologien, vorrangig Computern und Internet, geschult sind, sondern vor allem auch darum, dass sie mit Medientechnologien kompetent und didaktisch reflektiert umgehen können. ${ }^{31}$ Laut KMK heißt dies konkret, ,,dass Lehrkräfte digitale Medien in ihrem jeweiligen Fachunterricht professionell und didaktisch sinnvoll nutzen sowie gemäß dem Bildungs- und Erziehungsauftrag inhaltlich reflektieren können." ${ }^{32}$

Um ein pädagogisches Konzept für die Nutzung von Medien zu entwickeln, muss die Lehrkraft selbst erst einmal verstehen, welche Chancen und Risiken der Medieneinsatz birgt und wie diese die angestrebten Ziele unterstützen oder aber erschweren können. Lehrende werden aufgefordert, sowohl über allgemeine Medienkompetenzen zu verfügen als auch in ihrem jeweiligen Fachbereich zu „Medienexperten“ zu werden. ${ }^{33}$ Im Kontext des Arabischunterrichts würde dies also bedeuten, dass die Lehrkraft sich zuerst allgemeines medienpädagogisches Wissen und im Weiteren auch spezifische Kenntnisse und Ideen zum sinnvollen, pädagogisch wertvollen Einsatz von Medien im Arabischunterricht aneignen muss, bevor sie diese dann an Schüler*innen verschiedener Altersklassen und Sprachniveaus vermitteln kann. Arabischlehrer*innen aus Großbritannien berichten beispielsweise, dass sie sich selbst nicht fähig fühlen, Internetrisiken einzuschätzen und sich deshalb nicht trauen, mehr neue Medien im Unterricht zu verwenden. ${ }^{34}$ Obwohl sie Ideen für die Verbindung von technologischen Mitteln und sprachlichen Inhalten haben, hält das fehlende Hintergrundwissen zu kritischem Mediengebrauch sie davon ab, diese tatsächlich umzusetzen.

\subsubsection{Fort- und Weiterbildungen im Bereich Medienbildung}

Bedenkt man zudem, dass technologische Möglichkeiten sich ständig weiterentwickeln und dass ein Großteil der Lehrkräfte immer noch zur Generation der sogenannten digital immigrants gehört, also jenen Personen, welche nicht mit neuen Technologien und vielfältigen medialen Möglichkeiten aufgewachsen sind,

\footnotetext{
${ }^{31}$ Vgl. Ständige Konferenz der Kultusminister der Länder in der Bundesrepublik Deutschland 2012, S. 7.

${ }^{32}$ Sekretariat der Kultusministerkonferenz 2016, S. 25.

${ }^{33}$ Vgl. ebd., S. 24f.

${ }^{34}$ Vgl. Snowden und Soliman, Rasha, Towler, Melissa 2016, S. 14.
} 
sondern sich diese erst im Erwachsenenalter angeeignet haben ${ }^{35}$, dann wird schnell klar, dass die Förderung der Medienbildung im Arabischunterricht vor allem einer robusten Aus- und Weiterbildung von Arabischlehrkräften im medienpädagogischen Bereich bedarf. Laut KMK sollte die Aufgabe der entsprechenden Lehrerausbildung innerhalb des Lehramtsstudiums von den zuständigen Hochschulen übernommen werden. ${ }^{36}$ Zudem sollte für bereits eingestellte Lehrer*innen eine adäquate Auswahl an Fort- und Weiterbildungen durch die Länder bereitgestellt werden, damit sie sich fundierte Medienkompetenzen aneignen und stetig weiterentwickeln können. ${ }^{37}$

Momentan befindet sich das aufblühende Feld der Arabischdidaktik in einer Situation, in der die geringe Verbreitung von Arabisch als Fremdsprache an deutschen Schulen und das Fehlen eines entsprechenden Arabischlehramtsstudiums einander bedingen. Da wenige Schulen Arabisch unterrichten, scheint es keinen Grund zu geben, der das Schaffen eines Lehrstuhls für Arabischdidaktik im deutschsprachigen Raum rechtfertigt: Wenn man keine Arabischlehrer*innen braucht, muss man auch keine ausbilden. Die Realität zeigt aber, dass Arabischlehrer*innen dennoch gebraucht werden, sowohl im herkunftssprachlichen Kontext als auch für die vereinzelten Wahlprogramme an deutschen Schulen. Selbst wenn Arabisch nur als zusätzliches Fach ein- oder zweimal pro Woche angeboten wird, sollte die Lehrkraft qualifiziert sein und eine adäquate Ausbildung genossen haben. Dementsprechend müssen Möglichkeiten geschaffen werden, um zukünftige Arabischlehrer*innen auszubilden und bereits tätige Arabischlehrer*innen weiterzubilden. Da die institutionelle Verankerung der Arabischdidaktik in Deutschland schwach ausgeprägt ist und es noch keine einschlägig ausgerichteten Professuren und Forschungsstellen gibt, sollten für die nahe Zukunft andere Weiterbildungsmöglichkeiten entwickelt werden.

\footnotetext{
${ }^{35}$ Vgl. Prensky 2001, S. 1-2.

${ }^{36}$ Vgl. Sekretariat der Kultusministerkonferenz 2016, S. 29.

${ }^{37}$ Vgl. ebd., S. 30.
} 


\subsubsection{Vernetzung von Arabischlehrer*innen}

In Deutschland gibt es aktuell zwei Vereine, die sich aktiv der Entwicklung des Bereichs Arabisch als Fremdsprache (AraF) widmen und die Lehrerbildung als wichtige Aufgabe verstehen. Zum einen besteht der 2016 gegründete Fachverband Arabisch e.V., der sich unter anderem die Unterstützung aller „Maßnahmen und Initiativen im Interesse einer wissenschaftlich fundierten Berufsvorbereitung zukünftiger Arabischlehrerinnen und -lehrer“38 zum Ziel gesetzt hat. Zu diesem Zweck bietet der Fachverband regelmäßig Tagungen und Seminare an, bei denen sich Arabischlehrer*innen und -dozent*innen über theoretische Ansätze für den Arabischunterricht austauschen und auch praktische Erfahrungsberichte und Vorschläge teilen können. Des Weiteren ist momentan der Verein der Arabischlehrer*innen e.V. in der Entstehung, welcher sich auch dafür einsetzen will, Schulungen für Arabischlehrer*innen im deutschsprachigen Raum anzubieten. Diese Initiativen, welche aktuell die einzigen Foren sind, die Arabischlehrer*innen im deutschsprachigen Raum vernetzen, werden in Zukunft maßgeblich zur Förderung von Medienbildung beitragen können und helfen, die Defizite eines fehlenden Arabischlehramtsstudiums auszugleichen.

Die KMK schlägt vor, online-basierte Fortbildungsangebote zu generieren, auf die Lehrer*innen flexibel zugreifen können. ${ }^{39}$ Sucht man im Internet nach bereits existenten Initiativen, findet man einige Angebote für Lehrer*innen im Bereich AraF, die vor Ort, oft in den USA, stattfinden. Hier seien beispielsweise die Professional Development Workshops erwähnt, welche die Qatar Foundation International in Kollaboration mit den in den USA bekannten Concordia Language Villages anbietet. Diese Initiative umfasst zwei Workshops vor Ort sowie drei Online-Seminare und richtet sich an Arabischlehrer*innen an Grundund Sekundarschulen sowie Dozent*innen in den USA und Kanada. Die Workshops beschäftigen sich mit Themen wie Lehrplanentwicklung und der Ausbildung interkultureller Kompetenzen. ${ }^{40}$ Ferner stößt man auf vergangene Initiativen, wie die Arabic Online Education-Internetseite der Aldeen Foundation, deren Workshop-Videos aus dem Jahr 2013 leider nicht mehr korrekt verlinkt und

\footnotetext{
${ }^{38}$ Fachverband Arabisch e.V. 2020.

${ }^{39} \mathrm{Vgl}$. Sekretariat der Kultusministerkonferenz 2016, S. 29.

${ }^{40}$ Vgl. Qatar Foundation International 2020.
} 
dementsprechend nicht mehr verfügbar sind. ${ }^{41}$ Zudem gibt es auf dem StreamingPortal YouTube einige Videos zum Thema AraF, beispielsweise Präsentationen von Mahmoud Al-Batal zu Themen wie Förderung der Schreibkompetenz. ${ }^{42}$ Diese Videos sind aber nicht sortiert oder in ein kohärentes Online-Seminar eingebettet, sodass auch hier eine kritische, bedürfnisbezogene Auswahl vonnöten ist. Von einer Online-Fortbildung kann man in dem Sinne also nicht sprechen.

Ein weiteres Ziel der Förderung von Medienbildung unter Arabischlehrer*innen in Deutschland könnte es also sein, Online-Fortbildungsangebote zu erstellen, durch welche sich Arabischlehrer*innen unterschiedlicher Schulen eigenständig und bedürfnisbezogen weiterbilden können. Um diese Ideen zu verwirklichen, müssen der Ausbau bestehender Netzwerke von Arabischlehrer*innen und die Kooperation mit internationalen Foren deshalb oberste Priorität haben. Laut KMK erfordert der zielgerichtete Einsatz von Medien im schulischen Unterricht vor allem den Austausch mit Kolleg*innen anderer Schulen sowie externen Partnern. ${ }^{43}$ Wenn Arabischlehrer*innen im Alleingang oder in kleineren Gruppen versuchen, ihre Medienkompetenz zu steigern und neue Wege für Medieneinsatz im Arabischunterricht zu gehen, dann ist das zwar nicht falsch, trägt aber kaum dazu bei, medienpädagogische Ansätze im Bereich AraF als Ganzes voranzubringen. Sinnvoller wäre es, Arabischlehrer*innen zu vernetzen und gemeinschaftlich über die Entwicklung neuer Unterrichtsansätze zu sprechen. Vor allem wenn man bedenkt, dass es im gesamten deutschsprachigen Raum keine Fachdidaktik Arabisch gibt, an die sich Arabischlehrer*innen wenden können und die sie im Auf- und Ausbau ihrer eigenen Medienkompetenzen unterstützen kann, müssen bestehen Netzwerke weiterentwickelt und gestärkt werden.

\subsubsection{Institutionelle Verankerung der Arabischdidaktik}

Für die fernere Zukunft könnte die tatsächliche Schaffung eines Lehrstuhls für Arabischdidaktik anvisiert werden, der sich die ganzheitliche Ausbildung zukünftiger Arabischlehrer*innen zum Ziel macht und dementsprechend auch medien-

\footnotetext{
${ }^{41}$ Vgl. Aldeen Foundation, Qatar Foundation International 2013.

42 Vgl. Al-Batal 2019.

${ }^{43}$ Vgl. Sekretariat der Kultusministerkonferenz 2016, S. 26.
} 
pädagogisches Wissen und Fähigkeiten vermittelt. Diese Zielsetzung rechtfertigt sich vor allem dadurch, dass die Folgen des Fehlens eines solchen Lehrstuhls im Ausland bereits konkret beobachtbar sind. Beispielsweise wurde im Jahr 2009 eine Befragung von 209 Arabischdozent*innen an amerikanischen Universitäten durchgeführt. Dabei zeigte sich, dass zwar ein Großteil von ihnen promoviert hat, wenn auch hauptsächlich in Literaturwissenschaften, unterdessen aber weniger als 18 Prozent der Dozent*innen insgesamt einen Abschluss in Arabischdidaktik besaßen. Al-Batal und Abdalla schlussfolgern daraus, dass viele der aktiv unterrichtenden Arabischlehrer*innen kein ausreichendes pädagogisches Wissen besitzen und unbedingt entsprechend geschult werden müssen. ${ }^{44}$ Bei einer weiteren Umfrage konnte Isleem feststellen, dass einige Dozent*innen mittlerweile an Fortbildungen für Arabischlehrkräfte teilgenommen haben, der Großteil aber weiterhin in akademischen Feldern wie Literaturwissenschaft, Islamwissenschaft oder Sprachwissenschaft ausgebildet ist, jedoch nicht in Fremdsprachen- oder Arabischdidaktik. ${ }^{45}$ Auch im Vereinigten Königreich sind viele der Arabischdozent*innen an Universitäten Muttersprachler*innen, die ihre Abschlüsse in Ländern der arabischen Welt gemacht haben. Die meisten von ihnen haben jedoch keine Ausbildung in Fremdsprachen- oder Arabischdidaktik genossen, weshalb es ihnen an theoretischem Hintergrundwissen für die Vermittlung des Arabischen als Fremdsprache fehlt. ${ }^{46}$

Obwohl diese Ergebnisse lediglich aus dem universitären Kontext stammen, können sie dennoch auf den schulischen Kontext bezogen werden. Wohl wissend, dass es im deutschsprachigen Raum noch kein Lehramtsstudium für Arabisch als Fremdsprache gibt, könnte man vermuten, dass auch hierzulande die meisten Arabischdozent*innen und -lehrer*innen Muttersprachler*innen sind, deren Fachwissen in Bereichen wie Literatur- oder Sprachwissenschaft liegt. Hierzu würde sich eine umfangreiche Studie empfehlen, um die tatsächliche Situation besser einzuschätzen. Im Kontext des Fremdsprachenunterrichts an deutschen Schulen würde man eine solche Situation jedenfalls für andere Sprachen nicht hinnehmen. Es wird erwartet, dass Spanisch-, Französisch- und Englischlehrer*innen entsprechend ausgebildet sind und die Sprache nicht nur beherrschen,

\footnotetext{
${ }^{44}$ Vgl. Abdalla und Al-Batal 2011-2012, S. 6.

45 Vgl. Isleem 2018, S. 242.

${ }^{46}$ Vgl. Featherstone 2018, S. 56.
} 
sondern auch vermitteln können. Die Idee, es sei ausreichend, Muttersprachler*in zu sein, um eine Sprache unterrichten zu können, verkennt die Komplexität von Fremdsprachendidaktik im Allgemeinen und die besonderen Qualifikationen, die Arabischlehrer*innen besitzen müssen. ${ }^{47}$ Außerdem führt sie dazu, dass Kenntnisse in Bereichen wie Medienpädagogik, welche normalerweise im Lehramtsstudium vermittelt werden, nicht als Teil des Profils von Arabischlehrer*innen verlangt werden, was sowohl die Lehrkräfte als auch die Schüler*innen vor Herausforderungen stellt.

${ }^{47}$ Vgl. Featherstone 2018, S. 57. 


\section{Medienbildung in Lehrplänen}

\subsection{Integration medienpädagogischer Ziele im Lehrplan}

Wie bereits festgestellt, ist ein Problem der Förderung von Medienbildung, dass man sie erst einmal als grundlegenden Aspekt des Bildungsauftrags deutscher Schulen anerkennen und sie dementsprechend in die Lehrpläne integrieren muss. Ein zweites Problem ist, dass man zwischen dem Lernen mit Medien und dem Lernen über Medien unterscheiden muss. Medienbildung wird oft nur als Ersteres, also als die Benutzung von Medien im Unterricht verstanden. ${ }^{48}$ Dass dieser Benutzung eine kritische Auseinandersetzung vorausgehen muss, ist vielen Lehrer*innen jedoch oft nicht oder nur unzureichend bewusst. ${ }^{49}$

Dieses fehlende Bewusstsein zeigt sich auch in den im Folgenden analysierten Lehrplänen, welche Medienbildung auf die unterschiedlichste Art und Weise interpretieren und definieren und die Nutzung digitaler Medien von kaum bis durchgängig in die Kompetenz- und Themenbereiche des Arabischunterrichts einbeziehen. Auch die KMK beklagt dieses Manko in ihrem Beschluss zur Medienbildung in der Schule mit deutlichen Worten:

Medienbildung als Lernen mit Medien und Lernen über Medien ist in den Lehrund Bildungsplänen der Länder zwar durchgängig ausgewiesen, allerdings unterscheiden sich Art, Umfang und Ausführlichkeit der Angaben deutlich. Wünschenswert wären die Aktualisierung und Akzentuierung der Medienbildung in den einzelnen Fächern und die Formulierung eigener fächerübergreifender Kriterien zur Medienbildung. ${ }^{50}$

Obwohl es in Deutschland nur einen Arabischlehrplan gibt, den man als Vergleichsgegenstand nutzen kann, nämlich den der Salzmannschule Schnepfenthal, kann man im internationalen Vergleich das gleiche Phänomen beobachten. Manche der in dieser Arbeit untersuchten Arabischlehrpläne erwähnen nicht nur Medienbildung als wichtigen Bestandteil des Lehrplans, sondern unterscheiden auch zwischen dem Lernen mit und über Medien und erläutern, wie

\footnotetext{
48 Vgl. Neuß 2005, S. 61.

${ }^{49}$ Vgl. Kampmann und Schwering 2017, S. 27.

${ }^{50}$ Ständige Konferenz der Kultusminister der Länder in der Bundesrepublik Deutschland 2012, S. 6.
} 
bestimmte Medien eingesetzt werden können, um sprachliche Kompetenzen in den vier Bereichen Lesen, Hören, Sprechen und Schreiben auszubilden. In starkem Kontrast dazu gibt es Lehrpläne, die weder das eine noch das andere vollbringen und den Bereich Medienbildung fast gänzlich auslassen. Im Mittelfeld liegen Lehrpläne, die Bezug auf Medieneinsatz im Allgemeinen und spezifische medialunterstützte Unterrichtsaktivitäten nehmen, aber der Reflexion dieser Medien und ihrer Vor- und Nachteile keine Beachtung schenken. Stellt man sich ein Zukunftsszenario vor, in dem die arabische Sprache sowohl auf Länder- als auch auf Bundesebene an Stellenwert gewonnen hat und als Fremdsprache an Schulen in verschiedenen Bundesländern unterrichtet wird, so kommt man nicht umhin zu erkennen, dass der Erfolg des Arabischunterrichts in gewichtigem Maße davon abhängen wird, ob und wie Medienbildung in den Lehrplan integriert wurde und ob unter den Arabischlehrplänen in dieser Hinsicht Übereinstimmung herrscht. Momentan sind Arabischlehrer*innen in Deutschland von diesem idealen Szenario jedoch noch weit entfernt, da es für schulischen Arabischunterricht fast keine Lehrpläne gibt und deshalb jede Lehrkraft die Unterrichtsvorgaben- und ziele mehr oder weniger selbst definieren muss. ${ }^{51}$

\subsection{Medienpädagogische Defizite in Deutschland}

Hinzu kommt die Tatsache, dass deutsche Lehrer*innen in verschiedenen internationalen Vergleichen immer wieder die hinteren Plätze belegen, was das Thema Medieneinsatz im Unterricht angeht. ${ }^{52}$ Zudem zeigt eine Auswertung verschiedener Schulleistungsstudien im Bereich Medienkompetenz, unter anderem der ICILS-Studie Computer- und informationsbezogene Kompetenzen von Schülerinnen und Schülern in der 8. Jahrgangsstufe im internationalen Vergleich von 2013, dass deutsche Schüler*innen bei der Verwendung neuer Medien durchweg mittelmäßig abschneiden und 30 Prozent der Befragten über nur rudimentäre Fähigkeiten im Umgang mit neuen Technologien verfügen. ${ }^{53}$ Dementsprechend mangelt es sowohl deutschen Schüler*innen als auch Lehrer*innen an Medienkompetenz, was das Thema innerhalb der deutschen Schullandschaft be-

\footnotetext{
${ }^{51}$ Vgl. Behzadi et al. 2019, S. 411.

52 Vgl. Eickelmann et al. 2014, S. $203 f$.

${ }^{53}$ Vgl. Friedrich-Ebert-Stiftung 2017, S. 7.
} 
sonders dringlich macht. Eine Möglichkeit, Medienkompetenz unter Lernenden und Lehrenden zu fördern, könnte also sein, von positiven Beispielen aus dem Ausland zu lernen. Dies soll aber nicht bedeuten, dass Lehrpläne aus anderen Ländern in Bezug auf Medienbildung automatisch besser sind. Deshalb werden die Lehrpläne aus dem Ausland kritisch analysiert und sowohl ihre Stärken als auch Schwächen im Bereich Medienbildung hervorgehoben. Ziel der folgenden Analyse soll es sein, Bereiche für mögliche Lehrplanänderungen und -erweiterungen zu identifizieren und positive Beispiele für den konkreten Medieneinsatz hervorzuheben, nicht aber, das Fehlen reflektierten Medieneinsatzes als grundsätzliches Scheitern des Lehrplanes oder des Arabischunterrichts an sich zu bewerten. Diese Erkenntnisse könnten zukünftig auch bildungspolitische Akteure in Deutschland bei der Formulierung neuer Lehrpläne leiten.

\subsection{Analyse von Lehrplänen für fremdsprachlichen}

\section{Arabischunterricht}

\subsubsection{Lehrplan der Salzmannschule Schnepfenthal ${ }^{54}$}

Wie in der Einleitung erläutert, wird in Deutschland die arabische Sprache vorrangig als Herkunftssprache oder als Wahlangebot unterrichtet. Erwähnenswert ist hier beispielsweise die Initiative der Professur für Arabistik der Otto-FriedrichUniversität Bamberg, welche einen Wahlkurs Arabisch mit zwei Wochenstunden an zwei Gymnasien in Bayern organisiert und durchführt. ${ }^{55}$ Zudem gibt es nach aktuellem Stand in ganz Deutschland momentan nur eine Schule, die Arabisch als Fremdsprache fest in ihrem Schulkonzept integriert und sie als Wahlpflichtfach für Nicht-Muttersprachler*innen unterrichtet: die Salzmannschule Schnepfenthal in Thüringen. Die Salzmannschule ist ein Spezialgymnasium für Fremdsprachen, welches sich die Förderung von besonders sprachbegabten Schülern*innen zum Ziel gesetzt hat. Dieses Ziel wird unter anderem dadurch angestrebt, dass alle Schüler*innen fünf Fremdsprachen aus einem Angebot von insgesamt neun Fremdsprachen belegen. Zu den Auswahlmöglichkeiten gehören auch die außer-

\footnotetext{
${ }^{54}$ Es wird darauf hingewiesen, dass die Verfasserin Schülerin an der Salzmannschule Schnepfenthal war und zum Zeitpunkt der Veröffentlichung dieser Masterarbeit dort als Lehrkraft tätig ist. ${ }^{55} \mathrm{Vgl}$. Otto-Friedrich-Universität Bamberg 2019.
} 
europäischen Sprachen Chinesisch, Japanisch und Arabisch, von denen eine gewählt wird. Die außereuropäische Sprache beginnt in der 6. Klasse und wird bis zur 10. Klasse fortgeführt. Arabisch kann fakultativ auch bis zur Abiturprüfung in der 12. Klasse weitergeführt werden. ${ }^{56}$

Für den Arabischunterricht an der Salzmannschule gibt es zwei Lehrpläne. Die erste Fassung für die Klassenstufen 6-10 wurde im Jahr 2002 erarbeitet. ${ }^{57}$ Eine zweite Fassung speziell für die Klassenstufen 11 und 12 wurde im Jahr 2007 ausgearbeitet. ${ }^{58}$ Die Fassung von 2002 beinhaltet zu Beginn allgemeine Grundsätze für den Unterricht an Thüringer Gymnasien, darauffolgend eine Ausführung der angestrebten Kompetenzen, Details zur Ausbildung der vier Kompetenzen Hören, Sprechen, Lesen und Schreiben und letztendlich Vorschläge für konkrete Unterrichtsinhalte und Bewertungskriterien. Einer der Ansprüche an den gymnasialen Unterricht in Thüringen ist die „Förderung von Kommunikation sowie von kritischem Umgang mit Informationen und Medien“. ${ }^{59}$ Des Weiteren wird der Vorschlag gemacht, Fachinhalte aus dem Arabischunterricht mit den Inhalten anderer Fächer zu verknüpfen. Ein mögliches Themengebiet ist neben Umweltund Gesundheitserziehung der Umgang mit Medien und Informationstechniken. Somit nimmt dieser Lehrplan gleich zu Beginn, wenn auch minimal, Bezug auf die Vorgaben der KMK. Die zwei genannten Vorschläge werden aber leider nicht weiter ausgeführt und finden sich im folgenden Teil des Lehrplans nicht wieder.

Im Lehrplan von 2002 wird Medienkompetenz zudem als Unterpunkt des Aspektes Methodenkompetenz erwähnt. Jedoch findet sich keine genauere Beschreibung der Medien, in deren Benutzung die Schüler*innen geschult werden sollen. Auch findet sich keine Auflistung relevanter Ziele, die im Bereich Medienkompetenz erreicht werden sollen. Es werden jedoch einige Aspekte erwähnt, die indirekt auf die Benutzung von Medien schließen lassen, zum Beispiel die Berücksichtigung „teilweise mediengestützter Textrepräsentation“60 in der Ausbildung von Hörverstehen oder auch die Zuhilfenahme von Computersoftware in der Erschließung von Texten. Diese Möglichkeiten werden aber nicht näher erläutert und auf die

\footnotetext{
${ }^{56}$ Vgl. Salzmannschule Schnepfenthal 2020.

${ }^{57}$ Vgl. Thüringer Kultusministerium 2002.

${ }^{58}$ Vgl. Thüringer Kultusministerium 2007.

59 Thüringer Kultusministerium 2002, S. 3.

${ }^{60}$ Ebd., S. 9.
} 
Verwendung und kritische Reflexion von Medien als Ziel an sich wird nicht näher eingegangen. Letztlich findet man in den genaueren Kompetenzbeschreibungen einige Punkte, die vermuten lassen, dass hier Medieneinsatz von Nutzen wäre. Dazu gehören beispielsweise das Verstehen von Nachrichten und Berichten im Bereich Hören und das Erstellen von SMS, E-Mails und Chatnachrichten als Teil der Schreibkompetenz. Zudem findet sich eine Übersicht möglicher inhaltlicher Schwerpunkte für die Klassenstufen 7/8 und 9/10. In dieser Übersicht wird unter anderem die Nutzung von Medien als Schwerpunkt im Themenbereich Alltagssituationen erwähnt.

Betrachtet man im Folgenden den Lehrplan für die Oberstufe von 2007, findet man deutlichere Bezüge zur Medienbildung. Im Bereich Methodenkompetenz sollen die Schüler*innen dazu befähigt werden, verschiedene Medien zur Informationsbeschaffung, -verarbeitung, und -darstellung sowie zur Selbstkontrolle zu nutzen. Obwohl hier nicht näher auf bestimmte Arten von Medien eingegangen wird, ist vorstellbar, dass verschiedene Textverarbeitungs- und Präsentationsprogramme sowie Internetseiten zum Einsatz kommen. Diese Vermutung wird im Folgenden im Bereich Schreibkompetenz bestärkt, in welchem Schüler*innen lernen sollen, mehrdimensionale digitale Texte, wie beispielsweise Flyer oder Werbetexte, zu erstellen. Überdies werden Schüler*innen in der Oberstufe mit authentischen Quellen zur Informationsaufnahme vertraut gemacht, welche Anlass für Hör-, Sprech-, Lese- und Schreibhandlungen bieten sollen. Auch in der Vermittlung der im Lehrplan festgelegten Themenbereiche muss die Lehrkraft „den Einsatz vielfältiger authentischer Texte, Medien und Materialien garantieren“. ${ }^{61}$ Wie sich in folgenden Lehrplänen zeigen wird, ist das Internet oft Hauptquelle für authentische Texte und die Bereitstellung authentischer Materialien findet beispielsweise durch Zuhilfenahme arabischer Nachrichtenseiten, Radioprogramme und Fernsehserien statt. Dies zeigt, dass der Einsatz neuer Medien sich nicht auf bestimmte Kompetenzbereiche beschränken muss, sondern zur Ausbildung aller sprachlichen Kompetenzen beitragen kann.

Obwohl konkrete Arten von Medien im Thüringer Lehrplan nicht genannt werden, wird dennoch deutlich, dass der Lehrkraft eine wichtige Rolle bei der Medien-

${ }^{61}$ Thüringer Kultusministerium 2007, S. 10. 
auswahl und -bereitstellung zufällt und die Schüler*innen diese zum Ausbau sprachlicher Mittel und zur Aufnahme neuer Informationen über die arabischen Länder verwenden sollen. Ein besonders wichtiges Ziel des Thüringer Lehrplans, welches im folgenden Vergleich nur in wenigen Lehrplänen erwähnt wird, ist die Befähigung der Schüler*innen, Medien entsprechend der Aufgabenstellung gezielt auszusuchen und effizient einzusetzen. In der Oberstufe ist nicht mehr nur die Lehrkraft verantwortlich für die Auswahl passender Medien, sondern die Lernenden beteiligen sich an diesem Prozess und verwenden Medien auch ohne Anleitung der Lehrkraft auf sinnvolle Weise. Zuletzt ist hervorzuheben, dass „Medien verstehen und sinnvoll nutzen“ ein obligatorischer Themenbereich ist. Obwohl dieser nicht weiter ausgeführt wird, liegt die Vermutung nahe, dass hier verschiedene Medien verglichen und kritisch reflektiert werden, was, wie von der KMK verlangt, zum Lernen über Medien nebst dem Lernen mit Medien beiträgt.

Im Vergleich der zwei Lehrpläne ist ein deutlicher Fortschritt im Bereich Medienbildung zu beobachten, was möglicherweise auch mit der dazwischenliegenden Unterrichtserfahrung von fünf Jahren zu tun hat. Sucht man im ersten Lehrplan nahezu vergeblich nach Bezügen zum Lernen über Medien, wird im zweiten Lehrplan deutlich, dass Medien nicht ausschließlich als Unterrichtsmittel verwendet werden, sondern auch selbst Unterrichtsgegenstand sein sollen. Zudem nimmt der zweite Lehrplan mehr Bezug auf die selbstständige Verwendung neuer Medien seitens der Schüler*innen. Trotzdem wird in diesem Lehrplan allgemein viel mehr Wert auf bestimmte Medienkompetenzen als auf die kritische Reflexion neuer Medien an sich gelegt.

\subsubsection{Lehrplan für das Lycée in Frankreich}

Schaut man sich Lehrpläne und Materialien für den Arabischunterricht in Frankreich an, findet man grundsätzlich mehr Bezüge zum Thema Medienbildung. Im Allgemeinen findet man auf der vom französischen Bildungsministeriums bereitgestellten Internetseite für Arabischunterricht ${ }^{62}$ einiges mehr an Ressourcen für

\footnotetext{
${ }^{62} \mathrm{Vgl}$. Ministère de l'Éducation nationale, de l'Enseignement supérieur et de la Recherche 2020.
} 
Arabischlehrer*innen, was auch nicht verwundert, da der Arabischunterricht an französischen Schulen etablierter ist als an deutschen Schulen. ${ }^{63}$

Im Arabischlehrplan für das Lycée $e^{64}$ findet sich gleich zu Beginn eine Ausführung zum Einsatz von Informations- und Kommunikationstechnologien im Unterricht. Hier wird hervorgehoben, dass neue Technologien unerlässlich sind, weil sie dabei helfen, authentische Texte verfügbar zu machen, und zur Sprachpraxis innerhalb und auch außerhalb des Unterrichts beitragen können. Des Weiteren können Schüler*innen durch den Einsatz des Internets auf authentisches Audiomaterial wie Nachrichten zugreifen und eigenständige Recherche auf arabischsprachigen Internetseiten betreiben. Darüber hinaus können durch den Einsatz digitaler Medien Kontakte mit Muttersprachler*innen auf der ganzen Welt geknüpft werden, beispielsweise durch die Teilnahme an Videokonferenzen und in Diskussionsforen. Zuletzt wird auch erwähnt, dass der Einsatz neuer Medien dazu beitragen kann, das eigenständige Lernen der Schüler*innen, vor allem außerhalb des Unterrichts, zu unterstützen, da sie durch Laptops, Tablets und andere mobile Endgeräte auch außerhalb der Unterrichtsaktivitäten auf Lehrmaterial zugreifen können.

Weiterführend werden die vier Kompetenzen und die entsprechenden Zielsetzungen näher beschrieben. Vor allem im Bereich Hörverstehen wird die Wichtigkeit von authentischen Materialien betont und dass diese durch verschiedene Medientypen bereitgestellt werden können. Ohne konkrete Beispiele zu nennen, werden unter anderem Audiomaterialien, Videos und Nachrichten aufgeführt. Zudem wird in diesem Lehrplan explizit erwähnt, dass die Schüler*innen mit der sprachlichen Variation innerhalb der arabischen Sprache vertraut gemacht werden sollen. Hiermit ist sowohl die Vermittlung von Kenntnissen im Hocharabischen als auch in verschiedenen Dialekten gemeint. Laut dem Lehrplan sollen Schüler*innen durch die Zuhilfenahme von Liedern, Romanen und Filmen die Dialekte der arabischen Welt kennenlernen. Vor allem die Verwendung von Filmen im Unterricht sollte hier hervorgehoben werden, da sie später noch einmal im Bereich „kulturelle Bereicherung“ als besonders wertvoll erwähnt wird, in welchem sie dazu dient, kulturelle Inhalte zu vermitteln und die Schüler*innen mit

\footnotetext{
${ }^{63}$ Vgl. Levallois 2009, S. 7.

${ }^{64} \mathrm{Vgl}$. Ministère de l'Éducation nationale 2010.
} 
Werken arabischer Künstler vertraut zu machen. Des Weiteren soll der Einsatz authentischer audio-visueller Materialien auch dazu beitragen, Inhalte aus Geschichte, Politik, Geografie und Soziologie zu vermitteln.

Im Vergleich zum Thüringer Lehrplan wird im Lehrplan für das Lycée mehr Bezug auf individuelle Medien genommen und es werden konkretere Einsatzfelder für digitale Medien erwähnt. Trotzdem findet sich nichts zum Bereich Medienbildung im Sinne vom Lernen über Medien, sondern lediglich ein Beispiel für den Einsatz bestimmter Arten von Medien, um Themenbereiche und Unterrichtsaktivitäten zu unterstützen. Um ein ganzheitliches Bild zu erhalten, empfiehlt es sich jedoch, auch andere Dokumente, die auf der Internetseite Langue et Culture arabes ${ }^{65}$ bereitgestellt werden, zu analysieren. Dort gibt es unter anderem ein Dokument namens Contenus Culturels et Linguistiques: Arabe ${ }^{66}$, welches konkrete Themenbereiche für den Arabischunterricht vorgibt, beispielsweise Feste und Traditionen, Sport und Freizeit und Schul- und Arbeitswelt. Einer der Themenbereiche heißt Medien und ist nochmals in Massenmedien und Werbung sowie Reklame unterteilt. Hier sollen sich die Schüler*innen unter anderem mit Fernsehprogrammen, Radiosendungen, Zeitschriften, Blogs und Diskussionsforen sowie den Nachrichten und Interviews beschäftigen. Auch die Themen Meinungsfreiheit und Zensur sollen hier eingebracht werden. Diese Auflistung lässt jedoch nicht erkennen, inwieweit diese Themen nur besprochen oder auch aktiv umgesetzt werden. Man kann aus diesem Dokument allein nicht erschließen, ob die Schüler*innen lediglich Gespräche in Online-Foren lesen oder ob sie auch aktiv daran teilnehmen oder gar über potenzielle Gefahren in diesen Foren sowie angebrachte Netiquette diskutieren. Es ist auch nicht zu erschließen, ob sie sich Fernsehprogramme nur anschauen oder auch über Fernsehkultur, beispielsweise die Bedeutung von Serien im Ramadan, sprechen und lernen, wie sie zwischen qualitativ hochwertigen und möglicherweise bedenklichen arabischen Sendungen unterscheiden können und wo diese im Internet und anderswo zu finden sind.

\footnotetext{
${ }^{65} \mathrm{Vgl}$. Ministère de l'Éducation nationale, de l'Enseignement supérieur et de la Recherche 2020.

${ }^{66}$ Vgl. Ministère de l'Éducation nationale 2009.
} 


\subsubsection{Lehrplan der Edmonton Public Schools in Alberta, Kanada}

Um ein vollständigeres Bild des fremdsprachlichen Arabischunterrichts auf der Welt zu bekommen, wurde im Rahmen dieser Masterarbeit beschlossen, die Suche nach Lehrplänen auch außerhalb Europas weiterzuführen und unter anderem Nordamerika mit einzubeziehen. Es zeigte sich nach näherer Recherche, dass eines der bekanntesten Arabischprogramme im schulischen Kontext in Kanada an den Edmonton Public Schools umgesetzt wird, einem Zusammenschluss von sechs staatlichen Schulen in Edmonton, der Hauptstadt der Provinz Alberta, welche zusammen alle Klassenstufen von der Vorschule bis zur 12. Klasse umfassen. Das Besondere am Konzept dieser Schulen ist, dass sie bilingualen Unterricht auf Englisch und Arabisch anbieten und es sich zum Ziel gesetzt haben, den Schüler*innen Kenntnisse und Fähigkeiten in beiden Sprachen zu vermitteln, damit sie beide eigenständig für kommunikative Zwecke verwenden können. Es wird jedoch deutlich hervorgehoben, dass Englisch oft die Muttersprache der Schüler*innen ist. Gleichwohl soll ihnen ab dem 5. Lebensjahr auch Arabisch beigebracht und parallel zur englischen Sprache verwendet werden. Ein wichtiges Ziel ist hierbei vor allem, interlinguistische und -kulturelle Vergleiche anzustellen und den Schülern*innen die Kulturen der arabischen Länder näherzubringen. ${ }^{67}$

Der Lehrplan für Arabic Language Arts Kindergarten to Grade $12^{68}$ besteht aus drei Teilen: General Language Component, Specific Language Component und Culture. Im ersten Teil werden allgemeine Ziele und Themenbereiche des Sprachunterrichts erläutert, welche auf alle unterrichteten Sprachen zutreffen, inklusive der Muttersprache Englisch. Im zweiten Teil werden die angestrebten Kompetenzen und sprachlichen und kulturellen Inhalte für den Arabischunterricht beschrieben. Im dritten Teil findet sich eine Ausführung der Kenntnisse, Fähigkeiten und Einstellungen, welche die Schüler*innen in Bezug auf sich selbst, die arabische Sprache und Kultur und die Weltgemeinschaft erlernen sollen.

Die Zielsetzungen sind so formuliert, dass immer ein generelles Ziel vorgegeben ist, welches dann durch spezifische Ziele näher erklärt wird. Im ersten Teil findet man beispielsweise General Outcome 2: „Students will listen, speak, read, write,

\footnotetext{
${ }^{67}$ Vgl. Edmonton Public Schools 2020.

${ }^{68}$ Vgl. Alberta Education 2018.
} 
view, and represent in Arabic to comprehend and respond personally and critically to oral, print, visual, and multimedia texts. “69

Hier wird die Verwendung von multimedialen Texten grundsätzlich mit allen vier Kompetenzen - Hören, Sprechen, Lesen und Schreiben - in Verbindung gebracht. Zudem soll es nicht nur darum gehen, diese Texte zu verstehen, sondern kritisch auf sie zu reagieren. Diese Zielsetzung wird dann weiter ausgeführt: Im Umgang mit Texten beispielsweise sollen die Schüler*innen in der Vorschule ausdrücken, welche Gefühle verschiedene mündliche, gedruckte, visuelle und multimediale Texte in ihnen auslösen. In der 4. Klasse sollen diese multimedialen Texte dann verwendet werden, um zu erkennen, wie sich die Lebenswirklichkeiten von Personen in verschiedenen Kulturkreisen unterscheiden. In der 9. Klasse sind die Schüler*innen dann imstande, kulturelle Marker wie Traditionen innerhalb Kanadas und der arabischen Welt aus multimedialen Texten $\mathrm{zu}$ erschließen. In den höheren Klassen 10-12 sollen sie zudem darüber reflektieren, wie Wortwahl und sprachliche Details in multimedialen Texten bestimmte Bedeutungen hervorrufen und wie sie auf verschiedene Rezipienten wirken.

Im sprachlichen Erkennen von Forms and Techniques beginnen die Schüler*innen der Vorstufe damit, verschiedene Texte zu lesen, zu hören und anzuschauen, um zwischen Realität und Fiktion zu unterscheiden. In der 6. Klasse sollen sie dann zwischen verschiedenen Genres multimedialer Texte unterscheiden können und sich in der 9. Klasse vor allem multimedialen Texten aktueller Popkultur zuwenden. In der Oberstufe wird erwartet, dass die Schüler*innen selbst multimediale Texte erstellen können, um zu kommunizieren.

Andere Zielsetzungen sind zum Beispiel im Bereich Discover and Explore, gemeinsam mit den Schüler*innen der Oberstufe eine Bandbreite an mündlichen, gedruckten, visuellen und multimedialen Texten zu erforschen und zu analysieren sowie einzelne Werke bestimmter Schriftsteller*innen, Künstler*innen und Regisseur*innen kennenzulernen. Außerdem sollen Schüler*innen der 6. Klasse sprachliches Material anhören und -schauen, um sprachliche Mittel zu extrahieren und ihre eigenen Ausdrucksformen im Arabischen zu verbessern. Im Bereich Hörverstehen wird des Weiteren angestrebt, dass die Schüler*innen in der 6. Klas-

${ }^{69}$ Alberta Education 2018, S. 7. 
se die wichtigsten Informationen längerer gesprochener Texte verstehen können, vor allem wenn es um ihnen vertraute Themenbereiche geht. In der 12. Klasse sollen sie dann so weit gekommen sein, dass sie auch Details längerer mündlicher Texte verstehen, selbst wenn die Themen teils neu und in ihnen unbekannten Sprechstilen vorgetragen sind. Hier stellt sich die Frage, um welche Arten von mündlichen Texten es sich handelt. Vorstellbar wäre alles Mögliche, von wissenschaftlichen Vorträgen bis hin zu Dokumentationen und journalistischen Berichten, politischen Reden oder auch Hörspielen. Zudem muss man sich als Lehrkraft fragen, wie und wo man längere, teils unbekannte Audiomaterialen erhalten kann - ein offensichtlicher Vorschlag wäre mittels des Internets. Zuletzt sollen die Schüler*innen auch ihre interkulturellen Kompetenzen erweitern, indem sie mit Muttersprachler*innen kommunizieren und interagieren, zum Beispiel durch Schüleraustausche, Brieffreundschaften und Teilnahme an kulturellen Veranstaltungen. Obwohl die Umsetzung solcher Unternehmungen in einer Stadt von der Größe und Diversität Edmontons komplett ohne technische Hilfsmittel durchaus vorstellbar ist, könnte man diesen Bereich zumindest als potenzielles Einsatzfeld für neue Medien wie Chat- und Diskussionsforen, E-Mail-Austausche und Videoanrufe betrachten.

Medienbildung und -kompetenz als solche kommen in diesem Lehrplan nicht vor und werden auch nicht ausdrücklich mit bestimmten sprachlichen Kompetenzen oder Zielsetzungen für den Unterricht in Verbindung gebracht. Außerdem werden keine konkreten Medien, wie zum Beispiel Zeitungen, Filme, Internetseiten oder Ähnliches genannt, sondern der Begriff multimedia texts wird lediglich mehrmals wiederholt. Was man sich darunter vorstellen kann und soll, bleibt offen. Auch ob es sich hierbei vor allem um authentische oder didaktisierte Texte handelt, bleibt ungeklärt. Es ist jedoch zu vermuten, dass in den höheren Klassenstufen mehr authentische Texte verwendet werden, um direktere kulturelle Bezüge zu schaffen und die Komplexität der Übungen, vor allem im Hören und Lesen, zu verstärken. Allgemein ist der Lehrplan aus Edmonton in eher vager Sprache gehalten, sodass man sich nichts oder alles vorstellen kann. Statt nach einem konkreten Bezug zu Medienkompetenzen zu suchen, empfiehlt es sich, diesen Lehrplan nach potenziellen Kompetenzbereichen zu erforschen, welche vom Einsatz neuer Medien profitieren würden. Hierzu gehören beispielsweise die direkte Erfahrung der 
arabischen Kultur, der Austausch mit Muttersprachler*innen, das Anhören längerer Audiotexte und das Kennenlernen medialer Produkte aktueller Popkultur. Zusammenfassend kann man sagen, dass es diesem Lehrplan sehr nützen würde, einen separaten Teil zum Thema Medienkompetenz hinzuzufügen, welcher Sinn und Zweck des Einsatzes neuer Medien im Unterricht erläutert, den Begriff multimediale Texte näher definiert und möglicherweise auch konkreten Bezug auf die Bereitstellung von authentischen Materialien durch neue Medien nimmt.

\subsubsection{Lehrplan der Fairfax County Public Schools in Virginia, USA}

Auch in den USA erfreut sich die arabische Sprache wachsender Beliebtheit. ${ }^{70}$ In Fairfax County im Bundesstaat Virginia wird als Teil des World Language Program unter anderem Arabisch als Fremdsprache an acht High-Schools angeboten, welche die 9. bis 12. Klasse umfassen. Für jede Klassenstufe gibt es einen inhaltlichen Lehrplan, der eine große Auswahl an Themenbereichen umfasst sowie jeweilige Unterrichtsaktivitäten und -ziele dazu. ${ }^{71}$ Des Weiteren findet man die Foreign Language Standards of Learning for Virginia Public Schools für angebotene Sprachen, welche nicht das lateinische Alphabet verwenden. ${ }^{72}$ Nimmt man diese Dokumente zusammen, erhält man einen guten Einblick in die Zielsetzungen des Arabischunterrichts, die Ausbildung der sprachlichen Kompetenzen und auch die Verwendung neuer Medien im Arabischunterricht.

Ein Aspekt, der innerhalb der Vorgaben für Public Schools in Virginia auffällt, ist der klar formulierte Zusammenhang zwischen neuen Medien und der Benutzung authentischer Texte:

An important component of learning a modern world language is using the language in the real world beyond the classroom setting. One way this can be accomplished is through integration of technology into the classroom, an important means of accessing authentic information in the target language and in

\footnotetext{
${ }^{70}$ Vgl. Looney und Lusin 2018, S. 3.

${ }^{71}$ Vgl. Fairfax County Public Schools 2020e.

72 Vgl. Board of Education Virginia Commonwealth 2014.
} 
providing students the opportunity to interact with other speakers of the language. ${ }^{73}$

Laut dieser Aussage ist eines der wichtigsten Ziele des Fremdsprachenunterrichts die Verwendung der Sprache außerhalb des Klassenzimmers. Um dieses Ziel zu erreichen, müssen die Schüler*innen mit authentischen Informationen über die Sprache und ihre Sprecher*innen in Berührung kommen. Dazu sollen neue Medien in den Unterricht integriert werden, um authentische Materialien und Erlebnisse ins Klassenzimmer zu holen. Der Lehrplan definiert culturally authentic materials als alle Texte, die von und für Muttersprachler*innen erstellt wurden und aus der Kultur selbst heraus entstanden sind. Oder anders gesagt: Texte, die von arabischsprachigen Personen mit Bezug auf die arabische Kultur - obwohl dieser Begriff auch näherer Definition bedarf - erstellt wurden und sich sprachlich und inhaltlich an andere Muttersprachler*innen richten. Dementsprechend kann man durchaus annehmen, dass immer dann, wenn im restlichen Lehrplan von der Verwendung authentischer Materialien gesprochen wird, auch der Einsatz neuer Medien eine wichtige Rolle spielt.

Der Lehrplan beschreibt die angestrebten Arabischkompetenzen auf vier Niveaus und einem zusätzlichen Niveau, welche mehr oder weniger dem Durchlaufen der Jahrgansstufen 9-12 entsprechen. Im Bereich Lese- und Hörverstehen sieht man einen deutlichen Fortschritt in den Fähigkeiten der Schüler*innen, welcher vorrangig auf dem Verstehen authentischer Inhalte basiert. So sollen in den ersten Jahren die Inhalte von Werbetafeln, Ansagen, Nachrichten und Reklamen verstanden werden. Später sollen die Schüler*innen dann sowohl Haupt- als auch Nebenaussagen aufgenommener Gespräche und kurzer Vorlesungen sowie Berichte und Anleitungen zu verschiedenen Themen verstehen. All diese Texte sollen nach Möglichkeit culturally authentic sein, also tatsächlich von und für Muttersprachler*innen erstellt. Obwohl hier nicht direkt vom Einsatz neuer Medien gesprochen wird, liegt die Vermutung nahe, dass diese authentischen Materialien wie oben beschrieben durch technologische Hilfsmittel bereitgestellt werden. Denkbar wären verschiedene Internetseiten, Werbespots auf Portalen wie YouTube, Berichte arabischer Nachrichtensender und vieles mehr. Diese Vermu-

\footnotetext{
${ }^{73}$ Board of Education Virginia Commonwealth, S. 12.
} 
tung bestätigt sich auch darin, dass die Bewertung der rezeptiven Fähigkeiten der Schüler*innen vorrangig an ihrem Verstehen gesprochener und geschriebener Sprache in verschiedensten Medien festgemacht wird.

Weiterhin sollen neue Medien laut Lehrplan dazu verwendet werden, Informationen über die arabische Welt zu suchen, vor allem um kulturelle Einblicke zu gewinnen. Unter dem Kompetenzbereich Interacting in School and Global Communities beispielsweise soll Internetrecherche durchgeführt werden, um die arabische Sprache und Kultur näher kennenzulernen. Danach sollen kulturelle Erkenntnisse, die durch Online-Quellen gewonnen wurden, unter Zuhilfenahme technologischer Hilfsmittel vorgestellt und erklärt werden. Zudem zielt der Unterricht in den späteren Jahren darauf ab, die Schüler*innen zur eigenständigen Suche nach Unterhaltungsmedien in arabischer Sprache zu motivieren und diese auch außerhalb des Klassenzimmers für die eigene Bildung zu nutzen. Innerhalb dieses Kompetenzbereichs wird außerdem die Wichtigkeit des Austauschs mit Muttersprachler*innen hervorgehoben. So sollen Schüler*innen unter anderem das Internet benutzen, um mit Muttersprachler*innen zu kommunizieren. Obwohl die genaue Verwendung des Internets hier nicht näher erläutert wird, könnte man sich den Einsatz von Diskussionsforen und Chaträumen, Sofortnachrichtendiensten wie WhatsApp oder Facebook Messenger oder auch Videochat-Programmen wie Skype oder Zoom vorstellen.

Betrachtet man die bisherigen Erkenntnisse aus diesem Lehrplan als Rahmen, der Vorschläge und Inspiration für die tatsächliche Unterrichtsdurchführung bietet, lohnt es sich, einen Blick auf die sehr detailliert ausgeführten Themenbereiche für die vier Arabischniveaus zu werfen. In fast allen Bereichen wird die Verwendung authentischer Texte vorausgesetzt und in den meisten deutlich auf die Verwendung neuer Medien hingewiesen:

Im ersten Schuljahr sollen die Schüler*innen im Internet Kataloge und Reklamen für Kleidung finden, einfache Ansagen und Werbespots aus der arabischen Welt verstehen, sich im Internet Speisekarten arabischer Restaurants anschauen und Bus- und Zugfahrpläne suchen. ${ }^{74}$ Im zweiten Schuljahr sollen sie im Internet Blogs und Artikel lesen, um mehr über den Alltag in der arabischen Welt zu lernen.

\footnotetext{
${ }^{74}$ Vgl. Fairfax County Public Schools 2020a.
} 
Zudem sollen sie sich über Gesundheit und Fitness sowie beliebte Freizeitaktivitäten informieren und sich Karten und Broschüren über verschiedene arabische Länder beschaffen und analysieren. ${ }^{75}$ Im dritten Schuljahr sollen die Schüler*innen Internetrecherche zu Wahlen und politischer Partizipation betreiben, sich durch Werbespots, Videos und Fernsehprogramme mit Jugendkultur vertraut machen und sich in den Medien zu Themen wie Artenschutz erkundigen. ${ }^{76}$ Im letzten Schuljahr werden deutlich komplexere Themenbereiche eingeführt, welche unweigerlich durch die Benutzung neuer Technologien unterstützt werden. Schüler*innen sollen sich Fernsehprogramme über Gesundheitsprobleme anschauen, arabische und amerikanische Filme vergleichen, im Internet über aktuelle Ereignisse und politische Veränderungen in den arabischen Ländern recherchieren und Nachrichtenberichte zu Themen wie Kriminalität, historischen Konflikten, und Stereotypen behandeln.

Zudem werden im letzten Schuljahr Medien und Technologie als separate Themenbereiche behandelt. Hier geht es zum Beispiel darum zu diskutieren, wie sich neue Technologien auf die Gesellschaft auswirken, wie sie zum Lösen gesellschaftlicher Probleme beitragen, wie sie zwischenmenschliche Kommunikation verändern und welche Rolle sie am Arbeitsplatz und in der Schule spielen. In Bezug auf die arabische Welt soll erforscht werden, welche Technologien wie verwendet werden, wie sie sich auf die arabische Sprache auswirken und wie sie zum Beispiel in Werbespots und Kunst zum Einsatz kommen. Im Themenbereich Medien soll deren Einfluss auf Berichterstattung, beispielsweise in Nachrichtensendungen und Zeitungsartikeln, diskutiert werden. Außerdem sollen die Schüler*innen mediale Inhalte kritisch reflektieren und selbst Stellung beziehen, sowie die Vor- und Nachteile moderner Massenmedien erkennen. Sie sollen darüber hinaus kulturelle Aspekte und Einflüsse in verschiedenen Medien erkennen und reflektieren und selbst authentische arabische Massenmedien finden, um ihre Inhalte mit denen amerikanischer Medien zu vergleichen. All dies soll letzten Endes auch dazu dienen, sich mit medialer Sprache vertraut zu machen und linguistische Besonderheiten zu erkennen und, wenn angebracht, selbst zu verwenden. ${ }^{77}$

\footnotetext{
${ }^{75}$ Vgl. Fairfax County Public Schools 2020b.

${ }^{76}$ Vgl. Fairfax County Public Schools 2020c.

${ }^{77}$ Vgl. Fairfax County Public Schools 2020d.
} 
Der Lehrplan aus Fairfax County zeichnet sich dadurch aus, dass er einen allgemeinen Rahmen für den gewinnbringenden Einsatz moderner Medien im Fremdsprachenunterricht bietet und diesen durch verschiedene Themenbereiche mit Bezug auf die arabische Sprache detailliert ausarbeitet. Außerdem werden im letzten Schuljahr viele Aspekte der Medienbildung einbezogen, da die Schüler*innen den Einsatz neuer Technologien kritisch reflektieren und sich eigene Meinungen über Medien und deren Einfluss auf ihr Leben bilden sollen. Man könnte hier jedoch kritisieren, dass diese Themenbereiche schon früher eingeführt werden sollten, um auch jüngeren Schülern medienpädagogische Inhalte zu vermitteln. Alles in allem wird aber in diesem Lehrplan deutlich, dass neue Medien eine wichtige Rolle im Arabischunterricht spielen und zur Vermittlung einer Bandbreite an Inhalten beitragen können.

\subsubsection{Lehrplan für New South Wales, Australien}

Den Arabischlehrplan für Kindergarten bis 10. Klasse für Schulen im Bundesstaat New South Wales in Australien ${ }^{78}$ könnte man vielleicht als Paradebeispiel unter den für die Masterarbeit analysierten Lehrplänen betrachten. Der Lehrplan ist in die weiteren Vorgaben für schulische Bildung im Bundesstaat New South Wales und die Vorgaben für den Fremdsprachenunterricht eingebettet. Er beginnt mit einer Auflistung der wichtigsten Kompetenzen, die Schüler*innen während ihrer schulischen Laufbahn erwerben sollen. Dazu gehört, dass sie sich produktiv, kreativ und souverän im Umgang mit Technologie zeigen und die Auswirkungen technologischer Neuerungen auf die Gesellschaft verstehen. ${ }^{79}$ Somit ist der australische Lehrplan der erste, der gleich zu Beginn konkret Bezug auf Medienbildung und Medienkompetenz nimmt. Schüler*innen sollen nicht nur in der Verwendung technologischer Hilfsmittel geschult werden, sondern auch deren potenzielle Auswirkungen verstehen. Des Weiteren verdeutlicht der Lehrplan, warum der Einsatz neuer Medien vor allem für den Fremdsprachenunterricht gewinnbringend ist: „The learning of languages is enriched through access to a

\footnotetext{
${ }^{78}$ Vgl. Board of Studies New South Wales 2003.

${ }^{79}$ Vgl. ebd., S. 5.
} 
variety of multimedia resources. When students can access diverse authentic contexts with ease and speed, the boundaries of the classroom are extended. " ${ }^{\circ 80}$

Genau wie bereits im Lehrplan für Fairfax County formuliert, soll der Medieneinsatz im Unterricht vor allem dazu dienen, die Grenzen des Klassenzimmers zu erweitern, die Sprache und Kultur, die im Fremdsprachenunterricht behandelt werden, in den Klassenraum zu holen und den Schüler*innen authentische Materialien verfügbar zu machen. Im Folgenden wird dann kurz zusammengefasst, welche spezifischen Medienkompetenzen von Schüler*innen der unteren Klassenstufen K-6 und der weiterführenden Klassenstufen 7-10 erwartet werden. In der Unterstufe sollen sie Text, Ton und Bilder verwenden, um Präsentationen auf Arabisch zu erstellen, sich Textverarbeitungsfähigkeiten mit Bezug auf die arabische Sprache aneignen und Sprachlernsoftware kennenlernen. Darüber hinaus sollen sie lernen, elektronische Informationen zu sammeln und zu interpretieren, sich über moralische Aspekte von technologischen Neuerungen bewusst werden und Wissen im Bereich Technikethik erwerben. ${ }^{81}$ Auch hier zeigt sich wieder ein klarer Unterschied zwischen Medienkompetenz und Medienbildung. Dieser Lehrplan ist der einzige unter den ausgewählten, der den Bereich Technikethik und seine Wichtigkeit für schulische Bildung erwähnt.

In den oberen Klassenstufen soll Technologie dann vor allem eingesetzt werden, um mit Muttersprachler*innen zu kommunizieren und dabei sowohl Lese- als auch Schreibkompetenzen zu verbessern. Hier ist anzunehmen, dass die Schüler*innen grundsätzlich im Schreiben mit Stift und Papier als auch im Schreiben mit arabischen Tastaturen angeleitet werden. Weiterhin sollen sie im Internet Informationen und Nachrichten über die arabischen Länder recherchieren und muttersprachliche Audiomaterialien im Internet finden, anhören und als Muster authentischer Sprachverwendung betrachten, um ihre eigenen rezeptiven und produktiven Fähigkeiten zu verbessern. ${ }^{82}$

Der Lehrplan ist in die Stages 1-5 und die zusätzliche Stage 5 Extension unterteilt. Sinn und Zweck der Stages ist es, die angestrebten Ziele und Kompetenzen dem tatsächlichen Unterrichtspensum anzupassen, welches sich unter den betreffenden

\footnotetext{
${ }^{80}$ Board of Studies New South Wales 2003, S. 20.

${ }^{81}$ Vgl. ebd., S. 20.

${ }^{82}$ Vgl. ebd., S. 21.
} 
Schulen und basierend auf der individuellen Fächerauswahl der Schüler*innen unterscheiden kann. Die erwarteten Fähigkeiten innerhalb der vier Kompetenzbereiche Sprechen, Schreiben, Lesen und Hören sowie in den zwei zusätzlichen Bereichen Making Linguistic Connections und Moving Between Cultures werden in Bezug auf die bestimmten Stages dann näher erläutert.

Ein Merkmal, das diesen Lehrplan auszeichnet, ist, dass die Kompetenzbereiche jeweils in Students learn about und Students learn to unterteilt sind. Students learn about bezieht sich auf das Wissen, dass die Schüler*innen durch Anleitung erwerben, während Students learn to beschreibt, wie die Schüler*innen dieses Wissen umsetzen und wozu sie eigenständig fähig sind. Die Kompetenzbereiche nehmen nicht unbedingt durchgängig Bezug auf die Verwendung neuer Medien, obwohl die Vermutung nahe liegt, dass wie im Lehrplan aus Fairfax County die Verwendung authentischer Texte mit dem Einsatz neuer Medien Hand in Hand geht und dementsprechend dort verstärkter Medieneinsatz zu erwarten ist. Trotzdem gibt es ein paar explizitere Beispiele:

In Stage 3 und Stage 4 lernen die Schüler*innen, wie neue Technologien zu Informationsaustausch und Kommunikation beitragen können und sollen dann selbstständig solche Technologien verwenden, um eigene Texte zu erstellen und Informationen zu vermitteln. Dies soll sowohl zur Verbesserung des Schreibens als auch des Sprechens beitragen. Des Weiteren sollen sie erkennen können, inwiefern technologische Neuerungen zur Evolution der arabischen Sprache beitragen und sie beeinflussen. Im Bereich Moving Between Cultures, welchen man als den Erwerb interkultureller Kompetenzen beschreiben könnte, lernen Schüler*innen der Stage 4 wie die Kulturen der arabischen Welt in Massenmedien und Filmen dargestellt werden. Folglich sollen sie dann dazu imstande sein, Stereotypen und Verallgemeinerungen in verschiedenen Medien zu erkennen. Außerdem wird ihnen beigebracht, wie sie kulturelle Informationen im Internet finden und sammeln können und welche ethischen Aspekte beachtet werden müssen, um andere Kulturen moralisch vertretbar und reflektiert darzustellen. Darauf folgt die Verwendung des Internets, um selbstständige Recherche über die arabische Welt zu betreiben und beispielsweise Werbeanzeigen, Videos, Rezepte, Speisekarten und arabische Literatur zu finden. Diese Zielsetzungen sind ausgezeichnete Beispiele für den Unterschied zwischen Medienbildung und Medienkompetenz und die Inte- 
gration beider in den Arabischunterricht. Die Schüler*innen werden im kritischen, moralisch angebrachten und reflektierten Umgang mit Medien angeleitet und lernen darauffolgend, wie sie verschiedene Medien für ihre eigenen Bildungszwecke auf sichere und effektive Art und Weise anwenden können.

In Stage 5 beinhaltet der Bereich Lesekompetenz das Lernen über die Errungenschaften neuer Technologien für die Kommunikation. Dieses Wissen wird dann verwendet, um via E-Mail und in Internetforen mit Muttersprachler*innen zu kommunizieren. Im Bereich Schreibkompetenz wird den Schüler*innen vermittelt, welche Hilfsmittel ihnen auch außerhalb des Klassenzimmers zur Verfügung stehen, um ihre sprachlichen Mittel zu erweitern. Folglich sollen die Schüler*innen Internetseiten, Wörterbücher und authentische Texte im Internet finden und für ihr selbstständiges Lernen verwenden. Um ihre interkulturellen Kompetenzen zu erweitern, befassen sich die Schüler*innen mit kulturellen Einstellungen und Überzeugungen und wie diese sich in verschiedenen Texten wiederfinden. Dies soll dazu dienen, kulturelle Einflüsse in Videos, Zeitungsartikeln, Filmen und Werbung zu erkennen und zu reflektieren. In Stage 5 Extension werden Medien vor allem eingesetzt, um das eigenständige Lernen außerhalb des Unterrichts zu fördern. So erfahren die Schüler*innen, wo sie authentische Audio- und Lesematerialien finden können, um sich arabische Radioprogramme und Nachrichtensendungen anzuhören und Sach- und Prosatexte auf verschiedenen Internetseiten zu lesen.

Zusammenfassend kann man sagen, dass dieser Lehrplan am deutlichsten Bezug auf Medienbildung und Medienkompetenz als separate Bereiche nimmt. Die Wichtigkeit des reflektierten Umgangs mit Medien wird mehrmals hervorgehoben und der Einsatz neuer Medien wird eindeutig mit bestimmten Kompetenzbereichen in Verbindung gebracht. Überdies werden gelegentlich mehrere Arten von Medien aufgezählt und es wird durchweg ersichtlich, welche Rolle die Lehrkraft einnimmt und wo die eigenständige Verwendung von Medien seitens der Schüler*innen beginnt. Zudem zeigt dieser Lehrplan, wie Medien nutzbringend im Unterricht selbst, aber auch außerhalb des Unterrichts eingesetzt werden können und dass sie zur Ausbildung aller Kompetenzen beitragen. Erstaunlich ist vor allem die Tatsache, dass dieser Lehrplan, obgleich er aus dem Jahr 2003 stammt und somit nur unwesentlich aktueller als der erste Thüringer Lehrplan ist, dennoch viel deutlicher die Rolle von Medienbildung erkennt und erläutert. Eine mögliche Erklärung ist 
das ausdrückliche Interesse der australischen Regierung an verstärktem Medieneinsatz in der schulischen Bildung. ${ }^{83}$

\subsection{Erkenntnisse in Bezug auf die Vorgaben der KMK}

Nun stellt sich die Frage, inwiefern ein solcher Vergleich verschiedener Arabischlehrpläne der Umsetzung von Medienbildung im Arabischunterricht in Deutschland konkret dienen kann. Zum einen ist es interessant, zu sehen, inwieweit der Bereich Medienpädagogik überhaupt in den Lehrplänen erwähnt wird und ob man eine Unterscheidung zwischen dem Lernen über Medien und dem Lernen mit Medien erkennen kann. Zum anderen werden in einigen Lehrplänen konkrete Arten von Medien erwähnt, die in der Ausbildung bestimmter Kompetenzbereiche zum Einsatz kommen. Hier könnten sich auch Arabischlehrer*innen in Deutschland inspirieren lassen. Des Weiteren zeigen zum Beispiel der Lehrplan aus Fairfax County und die inhaltlichen Vorgaben aus Frankreich, wie Medieneinsatz die verschiedensten Themenbereiche zielgerichtet unterstützen kann. Auch hier könnte es sich lohnen, manche dieser Themenbereiche zu übernehmen und durch mediale Hilfsmittel zu bereichern.

Es ist jedoch wichtig zu bedenken, dass jegliche Neu- oder Umformulierungen von Arabischlehrplänen in Deutschland auch mit den Vorgaben der KMK übereinstimmen müssen und konkreten Bezug auf deren Vorstellungen zur Umsetzung von Medienbildung im Unterricht nehmen sollten. Deshalb lohnt es sich an dieser Stelle, die erwähnten Beispiele für Medienbildung und -kompetenz aus den Lehrplänen mit den von der KMK angestrebten digitalen Kompetenzen zu vergleichen, um zu sehen, inwieweit diese übereinstimmen und dementsprechend auch für den Arabischunterricht im deutschsprachigen Raum übernommen werden könnten.

Basierend auf der Auswertung verschiedener Studien zur Medienbildung in Deutschland und im Ausland, erstellte die Kultusministerkonferenz die Liste der Kompetenzen in der digitalen $W^{8}{ }^{84}$, um zu konkretisieren, welche Fähigkeiten Schüler*innen vermittelt werden sollen. Sie beinhaltet sechs Kompetenzbereiche:

\footnotetext{
${ }^{83}$ Vgl. Australian Trade and Investment Commission 2017.

${ }^{84} \mathrm{Vgl}$. Ständige Konferenz der Kultusminister der Länder in der Bundesrepublik Deutschland 2016.
} 
1. Suchen, Verarbeiten und Aufbewahren, 2. Kommunizieren und Kooperieren, 3. Produzieren und Präsentieren, 4. Schützen und sicher Agieren, 5. Problemlösen und Handeln, 6. Analysieren und Reflektieren. ${ }^{85}$ Laut KMK lohnt es sich, bestehende Lehrpläne dahingehend zu überprüfen, welche Beiträge sie hinsichtlich des Kompetenzrahmens bereits leisten und welche Anforderungen noch ergänzt werden müssen. ${ }^{86}$

Vergleicht man die Beispiele aus den Lehrplänen mit den laut KMK angestrebten digitalen Kompetenzen, wird deutlich, dass viele der genannten Bereiche schon umgesetzt werden. Die Thüringer Lehrpläne zum Beispiel verlangen die Verständigung auf Arabisch via SMS, Chatnachrichten und E-Mails, wodurch die Schüler*innen lernen, mit Hilfe verschiedener digitaler Möglichkeiten zu kommunizieren. Zudem sollen sie Medien selbstständig auswählen und anwenden, was sie dazu befähigt, digitale Werkzeuge bedarfsgerecht einzusetzen. Im Lehrplan für das Lycée wird Bezug auf Meinungsfreiheit und Zensur genommen, was dazu beiträgt, die Schüler*innen mit der Bedeutung von digitalen Medien für die politische Meinungsbildung vertraut zu machen und ihnen zu vermitteln, wie Mediennutzung zu gesellschaftlicher Teilhabe beitragen kann. Im Lehrplan aus Kanada wird zudem erwartet, dass die Schüler*innen selbst multimediale Präsentationen und Texte erstellen und sprachliche Mittel aus verschiedenen Medien extrahieren, um ihre Arabischkenntnisse zu erweitern und zu verbessern. Dadurch lernen die Schüler*innen, wie sie eine mediale Produktion planen, gestalten und präsentieren können und wie Informationen und Inhalte aus digitalen Medien in ihr bestehendes Wissen integriert werden können. Im Lehrplan aus Fairfax County zeigt sich mehrmals die Bedeutung von Medien im Bereich Suchen und Verarbeiten, da die Schüler*innen in verschiedenen digitalen Umgebungen nach Informationen über die arabische Welt suchen sollen. Des Weiteren gehören zu ihrer Recherchearbeit, wie von der KMK verlangt, das Analysieren, Interpretieren und Reflektieren digitaler Informationen. Außerdem werden die Schüler*innen ermutigt, digitale Lernmöglichkeiten zu finden und individuelle Lernressourcen zu organisieren und zu nutzen. Auch im australischen Lehrplan werden die Schüler*innen dazu angehalten, Informationen zu suchen, weiterzuverarbeiten und durch Zuhilfenahme

\footnotetext{
${ }^{85}$ Die vollständige Liste ist verfügbar unter https://www.kmk.org/fileadmin/Dateien/pdf/PresseUndAktuelles/2017/KMK_Kompetenzen___Bildung_in_der_digitalen_Welt_Web.html ${ }^{86}$ Vgl. Sekretariat der Kultusministerkonferenz 2016, S. 19.
} 
verschiedener Medien zu präsentieren. Des Weiteren sollen sie via Internetforen mit Muttersprachlerin*innen kommunizieren. Darüber hinaus hebt dieser Lehrplan die Wichtigkeit von Technikethik hervor und verlangt von den Schüler*innen, ethische Prinzipien der Kommunikation zu kennen und kulturelle Vielfalt in digitalen Umgebungen zu berücksichtigen, zum Beispiel durch Diskussionen zu kultureller Repräsentation und Stereotypen in Medien. Im Folgenden werden sie auch dazu angehalten, ihren eigenen Mediengebrauch zu reflektieren und gegebenenfalls zu modifizieren sowie digitale Technologien für soziales Wohlergehen zu nutzen.

Obwohl dies nur eine Auswahl einiger digitaler Kompetenzen ist, die in den verschiedenen Lehrplänen Beachtung finden, wird deutlich, dass keiner der Lehrpläne alle digitalen Kompetenzbereiche umfasst und dass es viel Spielraum für die Ausbildung digitaler Kompetenzen gibt. Dies ist auch nicht unbedingt problematisch, denn auch die KMK erwartet nicht, dass ein einziger Fachlehrplan jeden der aufgelisteten Bereiche umsetzt, sondern lediglich, dass jedes Fach „für seine fachbezogenen Kompetenzen Bezüge und Anknüpfungspunkte zu dem Rahmen“"87 definiert. Außerdem ist zu bedenken, dass nicht alle Kompetenzen sinnvoll im Arabischunterricht trainiert werden können und dass ihre Umsetzung in anderen Schulfächern mehr Sinn ergibt. Dazu gehört vor allem der Bereich „Schützen und sicher Agieren“, welcher vorrangig Themen wie mediale Abhängigkeit, Datenschutz und Umweltauswirkungen digitaler Medien behandelt. Würde man die hier analysierten Lehrpläne jedoch zusammenfügen, beziehungsweise ihre verschiedenen Ansätze für die Vermittlung von Medienkompetenzen in einen neuen Arabischlehrplan übernehmen, dann hätte man bereits eine Vielzahl der von der KMK geforderten Kompetenzen einbezogen und eine solide Basis für einen Arabischlehrplan geschaffen, der Medienbildung und -kompetenz als wichtig anerkennt und umsetzt.

\footnotetext{
${ }^{87}$ Sekretariat der Kultusministerkonferenz 2016, S. 20.
} 


\section{Anwendungsbeispiele für digitale Medien im Arabischunterricht}

\subsection{Theorie und Praxis}

Der Vergleich der Lehrpläne zeigt, inwiefern Medienbildung in die Fachplanung einbezogen wird und ob, beziehungsweise wie, medienpädagogische Aspekte von Anfang an als Teilziel des Arabischunterrichts betrachtet werden. Jedoch ist es wichtig, zu bedenken, dass ein Lehrplan lediglich ein Rahmen von Vorgaben ist, die aber oftmals nicht tatsächlich so umgesetzt werden. Zudem ist die Verwirklichung der Ziele und Aktivitäten, die im Lehrplan beschrieben werden, abhängig von der Lehrkraft und ihrer Unterrichtserfahrung, den Lehrmaterialien, schulinternen Vorgaben, Absprachen mit Kolleg*innen und weiteren Faktoren. ${ }^{88}$ Aus dem Lehrplan allein kann man nicht erschließen, ob dieser immer noch genauso im Unterricht umgesetzt wird, ob er überhaupt jemals genauso umgesetzt wurde und wie sich das tatsächliche Unterrichtsgeschehen von den Zielsetzungen und Kompetenzbeschreibungen im Lehrplan unterscheidet. Obwohl die Vorgaben aus Fairfax County und New South Wales beispielsweise den Eindruck erwecken, dass sowohl der Medienbildung als auch der Vermittlung von Medienkompetenzen große Wichtigkeit beigemessen wird, ist es ohne direkte Nachfrage bei den Arabischlehrer*innen und -schüler*innen oder Hospitation im Unterricht nicht möglich, die medienpädagogische Realität des Unterrichts einzuschätzen. Dementsprechend können die Lehrpläne nur einen Teil des Gesamtbildes zeigen und es lohnt sich, wenn möglich, den direkten Erfahrungsaustausch mit Arabischlehrer*innen zu suchen.

Um einen potenziellen Arabischlehrplan für den Arabischunterricht an deutschen Schulen mit Leben zu füllen, und die darin enthaltenen theoretischen Vorgaben zur Stärkung medienpädagogischer Ansätze in die Praxis umzusetzen, benötigen Arabischlehrer*innen konkrete Anwendungsbeispiele für den gewinnbringenden Einsatz neuer Medien im Arabischunterricht, die ihnen als Inspiration und Ausgangsbasis dienen können. $\mathrm{Zu}$ diesem Zweck soll im folgenden Teil der vorliegenden Forschungsarbeit eine Auswahl von Beispielen für den nutzbringenden

\footnotetext{
${ }^{88}$ Vgl. Vollstädt et al. 1999, S. 28.
} 
Medieneinsatz im Arabischunterricht aus verschiedenen Ländern und Bildungskontexten, vorrangig amerikanischen Universitäten, vorgestellt werden. Obwohl die Fragestellungen dieser Masterarbeit sich auf den deutschsprachigen schulischen Kontext beziehen, führt der Mangel an empirischem Material aus den deutschsprachigen Ländern dazu, dass Institutionen in den USA und anderen Ländern Hauptquellen für Erfahrungsberichte aus dem Arabischunterricht sind. Hinzu kommt, dass einige Beispiele bereits etwas überholt sind, aber aufgrund mangelnder Studien zur Verwendung digitaler Medien im Arabischunterricht dennoch hinzugezogen wurden. Bei den universitären Beispielen ist zu bedenken, dass Student*innen einerseits verschiedene Vorkenntnisse und Interessen haben als Schüler*innen, andererseits aber auch mit dem Erlernen der arabischen Sprache von ganz vorne beginnen und dementsprechend, trotz ihres fortgeschritteneren Alters, „banale“ Themenbereiche wie Tagesroutine, Freizeitaktivitäten, Familie und Freunde behandeln. In der folgenden Analyse wird untersucht, welche sprachlichen Ziele und Kompetenzen durch den Einsatz verschiedener Medien erreicht werden sollen. Diese Erkenntnisse sollen dann in Kombination mit den Erkenntnissen aus den Lehrplänen dazu dienen, potenzielle Anwendungsbereiche für neue Medien im Arabischunterricht zu skizzieren. Weiterhin wird hervorgehoben, ob es sich bei den genannten Medien um authentische Materialien oder Bildungsmedien handelt, um näher zu erforschen, welche Art von Medium für welche Zwecke besonders sinnstiftend scheint.

\subsection{Was sind authentische Texte?}

Fast alle der verglichenen Lehrpläne nehmen ausdrücklich Bezug auf die Verwendung authentischer Texte im Arabischunterricht und verbinden diese oft automatisch mit der Verwendung neuer Medien. Folglich stellt sich die Frage, was genau authentische Texte sind. Laut der allgemein anerkannten Definition von Galloway handelt es sich bei authentischen Materialien um geschriebene und mündliche Mitteilungen, die von Mitgliedern einer Sprach- und Kulturgruppe für andere Mitglieder derselben Sprach- und Kulturgruppe erstellt wurden. ${ }^{89}$ In Bezug auf den Arabischunterricht könnte man authentische Texte somit als alle geschrie-

${ }^{89}$ Vgl. Galloway 1998, S. 133. 
benen und gesprochenen Texte auf Arabisch, welche von Muttersprachler*innen für Muttersprachler*innen erstellt wurden, definieren. Al-Batal und Glakas schlussfolgern aus ihrer Befragung von Arabischlernenden an der University of Texas at Austin im Jahr 2015, dass authentische Texte einen wichtigen Stellenwert im Arabischunterricht haben, da sie Einblicke in die Kultur und soziokulturelle Realität von Arabischmuttersprachler*innen geben. ${ }^{90}$ Und obwohl es vor allem für Lernende mit Anfängerkenntnissen schwer sein kann, authentische Materialien zu verstehen, wird gleichwohl oft angeraten, dass man Schüler*innen so früh wie möglich an authentische arabische Texte heranführt. ${ }^{91}$

Die Erfindung des Internets hat dazu geführt, dass Millionen authentischer Texte auf Knopfdruck verfügbar sind, was auch von Fremdsprachenlehrer*innen als großer Vorteil angesehen wird - eine Fülle an authentischen Audiomaterialien, Videos und Lesetexten, die auf verschiedensten Internetseiten zu finden sind. ${ }^{92}$ Dies erklärt auch die Verbindung zwischen authentischen Texten und neuen Medien: Arabische Filme, Serien, Lieder, Artikel, Videos, Reportagen, Nachrichten und mehr können durch das Internet ohne großen zeitlichen oder finanziellen Aufwand im Unterricht und auch außerhalb bereitgestellt werden.

\subsection{Was sind Bildungsmedien?}

Die KMK definiert Bildungsmedien als ,speziell für Unterrichtszwecke aufbereitete Medien und Lernumgebungen mit konkretem Alltagsbezug für den Einsatz in allgemeinbildenden Schulen."93 Bildungsmedien vermitteln altersgerecht aufbereitete und didaktisierte Inhalte und umfassen beispielsweise Lehrbücher, Filme, Musikstücke oder Bildungssoftware. Anders ausgedrückt könnte man Bildungsmedien im schulischen Kontext auch als Gegenteil von authentischen Medien beschreiben. Genau wie authentische Texte können sie in gedruckter oder digitaler Form verfügbar sein. Ein großer Vorteil von Bildungsmedien im digitalen Zeitalter ist, dass sie sich nicht nur auf ein Medium beschränken müssen, wie es zum Beispiel bei einem Buch, Arbeitsblatt oder Lied der Fall ist, sondern dass man

\footnotetext{
${ }^{90}$ Vgl. Al-Batal und Glakas 2018, S. 273.

${ }^{91}$ Vgl. Stevens 2006, S. 256.

92 Vgl. ebd., S. 257.

${ }^{93}$ Sekretariat der Kultusministerkonferenz 2016, S. 31.
} 
vor allem durch die Entwicklung von Bildungssoftware und Internetseiten multimediale Lernumgebungen erstellen kann, welche verschiedene Elemente kombinieren. Somit können Bildungsmedien in der heutigen Zeit auch viel individualisierter gestaltet und eingesetzt werden und sich zielgerichteter an den Bedürfnissen und Interessen der Schüler*innen orientieren. ${ }^{94}$ Bildungsmedien für den Arabischunterricht können eine Bandbreite an sprachlichem Input bieten, zum Beispiel durch die Kombination audiovisueller Texte und interaktiver Übungen.

Die KMK verdeutlicht in ihren Vorgaben zur Nutzung neuer Medien im Unterricht, dass keinesfalls ausschließlich Bildungsmedien oder authentische Medien eingesetzt werden müssen, sondern dass das Zusammenspiel beider bestimmte Lehrund Lernprozesse deutlich bereichern kann. Letzten Endes ist es der Lehrkraft überlassen, zu wählen, wann welche Art von Medium den größten Beitrag zum Unterrichtsgeschehen leisten kann. Hier bietet es sich auch an, die Schüler*innen selbst in den Entscheidungsprozess einzubeziehen. ${ }^{95}$ Dieser Aspekt wird sich mehrmals deutlich in den folgenden Beispielen zeigen, in denen die Erstellung bestimmter Bildungsmedien sich konkret an den Zielen und Interessen der Lernenden orientiert und ihre Verwendung oft in Verbindung mit authentischen Materialien geschieht.

\subsection{Neue Medien und die Varietäten der arabischen Sprache}

\subsubsection{Erstellung passgenauer Bildungsmedien}

An der Brigham Young University werden Hocharabisch und die Dialekte der Levante $^{96}$ gemeinsam unterrichtet. In diesem Falle bedingen sich Medienkonsum und Medieneinsatz im Unterricht gegenseitig. Die Lernenden sind daran interessiert, arabische Fernsehserien anzuschauen und, um sie zu verstehen, müssen die Student*innen beispielsweise den syrischen Dialekt oder ähnliche Dialekte beherrschen. Folglich müssen die Lehrkräfte den Lernenden zumindest grundlegende Kenntnisse der Umgangssprache vermitteln, auf die sie später aufbauen können. Um dies zu tun, greifen die Lehrenden an der Brigham Young University

\footnotetext{
${ }^{94}$ Vgl. Sekretariat der Kultusministerkonferenz 2016, S. $31 \mathrm{f}$.

${ }^{95}$ Vgl. ebd., S. 31.

${ }^{96}$ Gemeint sind hier die Dialekte Palästinas, Syriens, Jordaniens und des Libanon.
} 
auf Bildungsmedien, die in Jordanien erstellt wurden, zurück und entwickeln zusätzlich ihre eigenen audiovisuellen Medien, um die Auswahl an verfügbarem Input zu erweitern. ${ }^{97}$ Dieser Lösungsansatz ist verständlich, wenn man bedenkt, dass Arabischlehrkräfte immer wieder das Fehlen adäquater Materialien bemängeln und folglich auf ihre eigene Kreativität zurückgreifen müssen. ${ }^{98}$ Auch die KMK sieht einen großen Vorteil in der Möglichkeit, dass Lehrer*innen Bildungsmedien selbst gestalten können. Sie sind nicht mehr nur von professionellen Produzenten wie Schulbuchverlagen oder öffentlich-rechtlichen Sendeanstalten abhängig, sondern können Materialien konzipieren, die passgenau den Bedürfnissen ihrer Klasse entsprechen. Zudem können Schüler*innen viel mehr in diesen Prozess einbezogen werden und bei der Entwicklung von Medien für den Unterricht mithelfen. ${ }^{99}$ Dennoch ist zu bedenken, dass die Erstellung von Unterrichtsmedien durch die Lehrkraft, womöglich ohne Konsultation mit erfahrenen Verlagshäusern oder Entwicklern von Bildungsmedien, auch Risiken bergen kann. Beispielsweise können die politischen, gesellschaftlichen und religiösen Ansichten der Lehrkraft Einfluss finden. Bei der direkten Übernahme von Unterrichtsmaterialien aus arabischen Ländern oder anderen Lernkontexten sollte man zudem das ursprüngliche Publikum dieser Materialien bedenken und entscheiden, inwiefern die Inhalte tatsächlich relevant und angebracht sind. Es wäre deshalb erstrebenswerter, Unterrichtsmaterialien in Kollaboration mit anderen Personen aus dem Bildungsbereich $\mathrm{zu}$ erstellen, um Einheitlichkeit $\mathrm{zu}$ schaffen und bestimmte qualitative Kriterien festzulegen und konsequent einzuhalten.

\subsubsection{Arabischsprachige Fernsehserien}

Auch an der University of North Texas spielen Fernsehserien eine wichtige Rolle im Unterricht. Lernenden wurde beispielsweise aufgetragen, eine Folge einer arabischen Fernsehserie anzuschauen und sie anschließend gemeinsam im Unterricht zu besprechen. Hier fiel den Student*innen vor allem auf, dass in arabischen Serien häufig mehrere Dialekte zusammenkommen und ein sprachliches Kaleidoskop bilden. Diese Beobachtung führte zu einer Diskussion über die aktuelle sprachliche

\footnotetext{
${ }^{97}$ Vgl. Belnap 2018, S. 39.

${ }^{98}$ Vgl. Snowden und Soliman, Rasha, Towler, Melissa 2016, S. $13 f$.

${ }^{99}$ Vgl. Sekretariat der Kultusministerkonferenz 2016, S. 31.
} 
Realität in der arabischen Welt und dass man in neuen Medien verschiedene Sprachvarietäten erwarten sollte. Folglich wurden die Student*innen dazu angehalten, sich im Laufe ihrer Arabischstudien mit verschiedenen Varietäten vertraut zu machen, um arabische Unterhaltungsmedien nutzen zu können. ${ }^{100}$ Das Medium Fernsehserie wurde selbst zum Unterrichtsgegenstand, führte zu einer kulturellen und linguistischen Diskussion und motivierte die Lernenden dazu, ihre sprachlichen Kenntnisse zu erweitern. Dieses Beispiel veranschaulicht auch einige der Vorteile, welche die KMK der Begegnung mit dem Medium Film zuschreibt. Dazu gehören beispielsweise die Förderung ästhetischer Sensibilität, die Geschmacksund Urteilsbildung sowie die Erweiterung der individuellen Ausdrucksfähigkeit. ${ }^{101}$ Obwohl sich Filme und Fernsehserien in vielerlei Hinsicht unterscheiden, kann vor allem auch die Erweiterung der individuellen Ausdrucksfähigkeit durch den Einsatz von Fernsehserien im Arabischunterricht erzielt werden. Wie Najour verdeutlicht, diente das Interesse an den Inhalten der Serie als Ausgangspunkt für den Erwerb dialektaler Kenntnisse. Auch im Beispiel der Brigham Young University bedingen sich der Konsum arabischsprachiger Fernsehserien und der Erwerb umgangssprachlicher Mittel gegenseitig.

\subsubsection{Ausbildung von Sprachbewusstheit}

Laut Giolfo und Salvaggio tragen Informations- und Kommunikationstechnologien dazu bei, eine virtuelle Lernumgebung zu erstellen, die es den Lernenden ermöglicht, den gleichen sprachlichen Input wie Arabischmuttersprachler*innen zu erhalten. ${ }^{102}$ Gemeint sind hiermit beispielsweise authentische Videos und Hörtexte, in denen al-fuṣhā und al- 'ämmīya ${ }^{103}$ zugleich verwendet werden und die es den Lernenden erlauben, sich an diese für Muttersprachler*innen typische Sprechweise zu gewöhnen. Des Weiteren können Lernende beispielsweise ein Video in der Umgangssprache anhören und im Anschluss schriftlich Fragen dazu auf Hocharabisch beantworten. Dies könnte dazu beitragen, gleichermaßen Hörverstehen in

\footnotetext{
100 Vgl. Najour 2018, S. 301.

${ }^{101}$ Vgl. Ständige Konferenz der Kultusminister der Länder in der Bundesrepublik Deutschland 2012, S. 5.

${ }^{102}$ Vgl. Giolfo und Salvaggio 2018, S. 95.

103 Al-fuṣhā bezeichnet das moderne Hocharabisch und al- 'āmmīya die arabische Umgangssprache bzw. die arabischen Dialekte.
} 
den Dialekten und Schreibkompetenz auf Hocharabisch auszubilden und den Schüler*innen ein Bewusstsein für beide Varietäten zu vermitteln. ${ }^{104}$ Trentman stimmt dieser Ansicht zu und schlägt vor, Lernende entsprechende Videos und Ausschnitte aus audiovisuellen Medien analysieren zu lassen, damit sie feststellen können, inwiefern Hocharabisch oder die Dialekte überwiegen. Folglich können sie anhand des bestimmten Kontextes reflektieren, warum Hocharabisch oder Umgangssprache in manchen Situationen angebrachter ist, als in anderen, um sich so größere Sprachbewusstheit anzueignen. Dies soll nicht zuletzt auch dazu dienen, ihre eigenen sprachlichen Mittel zu erweitern und, je nach Anlass, passend mit Muttersprachler*innen zu kommunizieren. ${ }^{105}$ Diese Zielsetzung für den Einsatz moderner Medien deckt sich auch mit den Vorgaben der KMK, welche unter anderem Sprachbewusstheit als wichtige Kompetenz für den Fremdsprachenunterricht im Sekundarbereich II aufführt. ${ }^{106}$

\subsubsection{Varietäten des geschriebenen Arabisch}

Eine grundlegende Diskussion unter Arabischlehrer*innen ist, ob und wie man dialektale Kenntnisse vermitteln soll. ${ }^{107}$ Ein Argument für das Unterrichten arabischer Dialekte ist, dass die Umgangssprache mittlerweile für verschiedenste Aspekte alltäglicher Kommunikation verwendet wird - auch solche, die vor kurzer Zeit noch vom Hocharabischen dominiert wurden. ${ }^{108}$ Im Erfahrungsbericht der University of Arizona wird beispielsweise hervorgehoben, dass man die Umgangssprache vielerorts geschrieben sieht, zum Beispiel in Blogeinträgen, wo sie verwendet wird, um eine Bandbreite an Themen zu diskutieren und kommentieren. Obwohl auch die Lehrkräfte der University of Arizona anerkennen, dass Dialekte häufiger gesprochen als geschrieben werden, halten sie ihre Student*innen dazu an, geschriebenes 'āmmīya zu lesen und daraus zu lernen, beispielsweise durch die Analyse von Posts und Kommentaren in den sozialen Medien. ${ }^{109}$ Obwohl dieses

\footnotetext{
${ }^{104}$ Vgl. Giolfo und Salvaggio 2018, S. 103.

105 Vgl. Trentman 2018, S. 130.

${ }^{106}$ Vgl. Ständige Konferenz der Kultusminister der Länder in der Bundesrepublik Deutschland 2011, S. 4.

107 Vgl. Al-Batal 2018, S. 3.

108 Vgl. Al-Batal 2018, S. 8.

109 Vgl. Shiri und Joukhadar 2018, S. 158.
} 
Beispiel banal erscheinen mag, trägt es doch zu der wichtigeren Diskussion bei, wann und wo Umgangssprache angebracht ist und ob, beziehungsweise wie, man Lernende an die tatsächliche sprachliche Realität der arabischen Welt heranführen kann. Zudem könnte man sich vor allem im schulischen Kontext vorstellen, dass Schüler*innen, die im Alltag bereits verschiedene soziale Medien verwenden, an einer solchen Aktivität interessiert wären und sie als relevant für ihr eigenes Leben bewerten würden.

\subsubsection{Unterschiede in der Aussprache}

Egal ob in Bezug auf die arabischen Dialekte oder nur auf das Hocharabische, Schüler*innen müssen an Unterschiede in der Aussprache des Arabischen herangeführt werden, um die Sprache in ihrer Gesamtheit zu lernen. Selbst bei der Aussprache des Hocharabischen gibt es von Sprecher*in zu Sprecher*in Unterscheidungen, denen Schüler*innen ausgesetzt werden sollten. Hinzu kommen dann möglicherweise noch die Unterschiede zwischen Hochsprache und den Dialekten und die regionalen Unterschiede innerhalb der Umgangssprache eines arabischen Landes oder einer Region. Hierbei sind manche Aussprachekonventionen leichter zu lernen als andere. Laut Van Mol ist die Aussprache des Buchstaben ǧìm in verschiedenen Varietäten beispielsweise leichter zu erkennen und anzuwenden, als das Auslassen mancher Vokale innerhalb der Dialekte. ${ }^{110}$ Schüler*innen können vor allem durch Hörtexte und Videos mit der Bandbreite an Aussprachemöglichkeiten konfrontiert werden, um diese zu erkennen, einzuordnen und resultierende Worte und Sätze zu verstehen. Hörtexte könnte man auch ohne das Medium Internet finden und vorspielen. Jedoch ist nicht abzustreiten, dass man im Internet eine größere Auswahl an Hörtexten finden kann, welche in Sekundenschnelle bereitgestellt werden können und auch tagesaktuelle Themen behandeln.

${ }^{110}$ Vgl. Van Mol 2006, S. 307f. 


\subsubsection{Verstärkung der Authentizität sprachlicher Situationen}

An der University of New Mexico wurde den Lernenden aufgetragen, ein für Unterrichtszwecke erstelltes Video in ägyptischer Umgangssprache anzuschauen und im Unterricht gemeinsam zu diskutieren. Im Video wird einer Person auf Ägyptisch in einem Telefongespräch eine traurige Nachricht überbracht, auf welche die andere Person entsprechend reagiert. Die Lernenden sollten im Folgenden erkennen, welche Worte und Ausdrücke in einer solchen Situation angebracht sind und sich dann gegenseitig auf Skype anrufen, um ein eigenes Szenario zu kreieren, in welchem sie einander traurige Nachrichten überbringen und sprachlich passend reagieren. Zum einen wurde das Video verwendet, um das Unterrichtsthema zu präsentieren und dialektale Kenntnisse zu vermitteln. Zum anderen diente das Video als Ausgangsbasis für die zweite, medial-gestützte Aktivität, in welcher die Lernenden sich selbst in die Rollen der Personen im Video versetzen, und, statt einfach nur einen Dialog zu schreiben oder vorzuspielen, einen Anruf durchführen, um die sprachliche Situation so authentisch wie möglich nachzustellen. ${ }^{111}$ Was den letzten Punkt betrifft, könnte argumentiert werden, dass ein Rollenspiel vor der Klasse genauso effektiv gewesen wäre und es die Anwendung von Skype nicht unbedingt gebraucht hätte, um die sprachlichen Mittel zu üben und authentisch auf die Situation zu reagieren. Trotzdem lohnt es sich gewiss, eine solche Aktivität einmal auszuprobieren, um dadurch einen Telefonanruf statt eines persönlichen Gesprächs zu simulieren. Ein potenzieller Vorteil hiervon ist, dass sich die Lernenden beim Telefongespräch ohne Videofunktion nicht an Mimik und Gestik ihres Gesprächspartners orientieren können, sondern allein durch ihr Sprechen Informationen vermitteln und entsprechend reagieren müssen.

\subsection{Kommunikation, Vernetzung und Austausch}

\subsubsection{Sofortnachrichtendienste}

Laut Stevens sollten Arabischlehrer*innen das Chatten mithilfe von Sofortnachrichtendiensten als potenziell sinnvolle Unterrichtsaktivität in Betracht ziehen, da die direkte Kommunikation mit Muttersprachler*innen entscheidenden Einfluss

${ }^{111}$ Vgl. Trentman 2018, S. $121 \mathrm{f}$. 
auf die Motivation der Lernenden haben kann. Statt unauthentische sprachliche Situationen im Unterricht nachzustellen oder sich mit leblosen Materialien zu befassen, können Lernende ihre sprachlichen Mittel in muttersprachlichen Interaktionen testen und anwenden, was vor allem dazu führen kann, die Sprache auch über einen längeren Zeitraum hinweg lernen zu wollen. ${ }^{112}$ Überdies ist die digitale Kommunikation ein von der KMK festgelegtes Kompetenzziel, welches die Schüler*innen auch aktiv an der Gesellschaft teilhaben lässt. Zudem könnte die Verwendung von Sofortnachrichtendiensten eine Gelegenheit bieten, deren potenzielle Risiken im Unterricht gemeinsam zu besprechen und zu reflektieren. ${ }^{113}$

\subsubsection{Vernetzung von Lernenden - communities of practice}

Stevens erwähnt zudem, dass digitale Medien auch zur Vernetzung von Arabischlernenden untereinander beitragen können. Er sieht einen herausragenden Nutzen des Internets darin, dass sich sogenannte communities of practice, beispielsweise in Form von Diskussionsforen, bilden. Diese Gemeinschaften bestehen meist aus Lernenden, die sich im gleichen Bereich spezialisieren und gemeinsam ihre Kenntnisse und Fähigkeiten erweitern wollen. ${ }^{114}$ Durch das Internet können Schüler*innen solchen communities of practice spontan, unkompliziert und meist kostenlos beitreten und mit Gleichgesinnten in Kontakt treten. Für Arabischschüler*innen bedeutet dies, dass sie sich mit anderen Arabischlernenden auf der ganzen Welt vernetzen, Erfahrungen und Tipps austauschen und Links zu Nachschlagewerken, Internetseiten und authentischen Texten teilen können. Dadurch erweitert sich der Kreis der Mitstreiter*innen auf dem Weg zur Beherrschung der arabischen Sprache und kann unter anderem Frustration und Motivationsverlust vorbeugen. Deutschen Schüler*innen solche communities of practice vorzustellen und sie zu motivieren, sich mit anderen Lernenden $\mathrm{zu}$ vernetzen, kann ihnen verdeutlichen, dass es sich lohnt, Arabisch zu lernen und dass sie auf diesem Lernweg nicht allein sind.

\footnotetext{
${ }^{112} \mathrm{Vgl}$. Stevens 2006, S. 254f.

${ }^{113}$ Vgl. Ständige Konferenz der Kultusminister der Länder in der Bundesrepublik Deutschland 2016.

${ }^{114} \mathrm{Vgl}$. Stevens 2006, S. 256.
} 


\subsubsection{Schreiben mit E-Mail und Textverarbeitungsprogrammen}

Madhany widmet sich in seinem Erfahrungsbericht aus der University of Chicago zwei scheinbar banalen Medien der digitalen Welt und wie diese sinnstiftend in den Arabischunterricht einbezogen werden können: Textverarbeitungsprogramme und E-Mail. Sein Vorschlag ist es, Schüler*innen mit einfachen Textverarbeitungsprogrammen auf Arabisch schreiben zu lassen. Einerseits, um sie an die Verwendung der arabischen Tastatur heranzuführen und andererseits, um ihnen das Erstellen verschiedener Textsorten auf Arabisch zu vermitteln. Letzteres könnte auch mit Stift und Papier geschehen, aber wie Madhany betont, bieten digitale Textverarbeitungsprogramme ganz andere Möglichkeiten für das Überarbeiten von Texten und können zudem zur Ausbildung grundlegender Fähigkeiten in unserer digitalen Welt beitragen. Er erwähnt auch, dass Textverarbeitungsprogramme Schreibfehler anstreichen und Lösungsvorschläge anbieten können. Dies kann Schüler*innen helfen, sich ihrer Schreibfehler bewusster zu werden und durch die Nutzung der integrierten Wörterbuchfunktion auch andere passende Worte in Erwägung zu ziehen und ihren Wortschatz zu erweitern. ${ }^{115}$ Das Erstellen von E-Mails auf Arabisch und der Austausch dieser E-Mails entweder zwischen Schüler*innen und Lehrer*innen, unter der Schülerschaft oder auch mit Muttersprachler*innen bietet den Vorteil, dass die Lernenden auch außerhalb des Unterrichts auf Arabisch schreiben und lesen und dies zu Themen, die ihren Alltag und ihre Interessen betreffen. Überdies ist davon auszugehen, dass Schüler*innen bereits eigene E-MailKonten haben und in ihrem Alltag regelmäßig verwenden. Ähnlich wie bei der Verwendung von Nachrichtendiensten oder sozialen Medien, kann man sie hier in ihrer Lebenswirklichkeit abholen und ihre bereits erworbenen Kenntnisse weiter ausbauen. Darüber hinaus kann das Erstellen von E-Mails zu verschiedenen Themen und für verschiedene Adressaten dazu beitragen, zu erkennen, wann das Schreiben auf fuṣhā und das Schreiben auf ämmiyya angebracht ist und Lernende mit dem Lesen und Schreiben im Dialekt vertrauter machen. Außerdem benutzen immer noch einige Muttersprachler*innen Umschrift, wenn sie arabische E-Mails, Chatnachrichten oder SMS verfassen. ${ }^{116}$ Ein Beispiel ist arabizi, welches lateinische Buchstaben und Zahlen verwendet, um die arabischen Schriftzeichen aus-

\footnotetext{
${ }^{115}$ Vgl. Madhany 2006, S. 297.

116 Vgl. ebd., S. $298 f$.
} 
zudrücken. Diese Umschrift zumindest verstehen und lesen zu können, selbst wenn man sie selbst nicht aktiv beherrscht, ist eine wichtige Fähigkeit, um arabische Inhalte in den sozialen Medien zu verstehen.

\subsubsection{Vermittlung zwischen „West" und „Ost"}

Laut Van Mol sollte Lernenden vor allem das Arabisch der Medien beigebracht werden, vorrangig durch Zeitungsartikel und Berichte aus der arabischen Welt. Er sieht ein großes Problem darin, dass in der ,westlichen“ Berichterstattung über die arabische Welt selten mehr als nur ein paar arabische Quellen herangezogen werden und Gastbeiträge, Leitartikel und Kolumnen, welche die Meinungen und Ansichten von Muttersprachler*innen ausdrücken, oft ignoriert werden. Vielmehr werden Artikel aus anderen europäischen und „westlichen“ Ländern als Informationsquellen benutzt und in andere Sprachen übersetzt. Seiner Meinung nach liegt das Problem vor allem darin, dass es im „Westen“ nicht genügend Menschen gibt, die Arabisch auf (fast) muttersprachlichem Niveau beherrschen und Artikel schnell lesen, verstehen und weiterverarbeiten können und welchen auch sprachliche Nuancen nicht entgehen. Vor allem arabische Stimmen, die sich zu aktuellen Themen in ihrem Land oder der weiteren arabischen Welt äußern, werden oft nicht an ein westliches Publikum herangetragen und stattdessen findet man in der allgemeinen Berichterstattung europäische oder nordamerikanische Interpretationen der aktuellen Lage. Van Mol ist deshalb der Meinung, dass man Schüler*innen so schnell wie möglich an authentische Materialien heranführen muss, damit sie sich an synonyme Entsprechungen, die schiere Menge an Worten im Hocharabischen und an die medientypische Sprache gewöhnen und so zukünftig zur Vermittlung von Informationen und Perspektiven zwischen West und Ost beitragen können. ${ }^{117}$ Hierfür bietet das Internet einen großen Fundus an Videos, Radiobeiträgen und Zeitungsartikeln, die zum Unterrichten des medialen Arabisch verwendet werden können und auch wichtige soziokulturelle und politische Inhalte transportieren.

117 Vgl. Van Mol 2006, S. 310. 


\subsection{Angeleitete und eigenständige Recherche}

\subsubsection{Recherche mit geringen Arabischkenntnissen}

Im Vergleich der Lehrpläne wurde deutlich, dass das Internet unter anderem eingesetzt werden soll, um Recherche über arabischsprachige Länder zu betreiben und Informationen $\mathrm{zu}$ verschiedensten Themen zu finden, von Gesundheit und Sport bis Kunst und Jugendkultur. Ein weiteres Beispiel der University of New Mexico zeigt, wie Lernende an die Recherche auf arabischsprachigen Internetseiten herangeführt werden können. In einer Aktivität zum Thema Reisen wurden den Student*innen zuerst Vokabeln im Bereich Urlaub und Ferien beigebracht. Anschließend sollten sie eigenständig auf der Internetseite einer arabischen Reiseagentur recherchieren und bestimmte Informationen zu Preisen, Hotelangeboten und Aktivitäten für den Urlaub sammeln. Danach wurden die gesammelten Informationen verwendet, um eine Postkarte über einen fiktiven Urlaubsaufenthalt $\mathrm{zu}$ verfassen. Die Benutzung der Internetseite wurde mit traditionelleren Materialien wie Vokabellisten verbunden und sollte mehrere Kompetenzbereiche wie eigenständiges Recherchieren, Leseverstehen und Schreiben unterstützen. Außerdem konnte so der didaktisierte Teil der Vokabelvorstellung und -erklärung mit den authentischen Informationen auf der arabischsprachigen Internetseite verbunden werden. ${ }^{118}$ Obwohl es, wie zuvor erwähnt, schwierig sein kann, authentische Materialien in den anfänglichen Arabischunterricht einzubeziehen, zeigt dieses Beispiel, dass es dennoch möglich ist und dass selbst Lernende mit geringen Sprachkenntnissen, wie von der KMK vorgeschlagen, Recherche in verschiedenen digitalen Umgebungen durchführen können, um neue Informationen zu finden und zu analysieren. ${ }^{119}$

\subsubsection{WebQuests}

Um die Idee der eigenständigen Recherche weiter auszubauen, schlägt Stevens vor, das Internet zum Beispiel zum Erstellen von WebQuests zu nutzen. ${ }^{120}$ WebQuests

\footnotetext{
${ }^{118} \mathrm{Vgl}$. Trentman 2018, S. 123.

${ }^{119}$ Vgl. Ständige Konferenz der Kultusminister der Länder in der Bundesrepublik Deutschland 2016.

${ }^{120} \mathrm{Vgl}$. Stevens 2006, S. 259.
} 
werden häufig als „abenteuerliche Spurensuche im Internet“ oder als „virtuelle Schnitzeljagd“ beschrieben. Sie stellen Schüler*innen vor eine Problemstellung, die sie in Gruppen lösen müssen. Dazu stehen ihnen normalerweise die von der Lehrkraft erstellte WebQuest-Internetseite mit den entsprechenden Links und Informationen zur Verfügung, sowie andere Materialien wie Bücher und CDs. Um das vorgegebene Problem zu lösen, müssen sie auf verschiedenen Internetseiten recherchieren, Entscheidungen treffen, Informationen extrahieren und sinnvoll nutzen, um zum nächsten Schritt zu gelangen. Anschließend werden die Ergebnisse der Klasse präsentiert. Diese Präsentation soll idealerweise auch eine Reflexion des eigenen Suchprozesses beinhalten und die Schüler*innen für sinnvolle Internetnutzung sensibilisieren, was das Lernen mit dem Internet und das Lernen über das Internet und seine Anwendungsbereiche verbindet. ${ }^{121}$ WebQuests vereinen einige der laut KMK wichtigen Aspekte für schulische Medienbildung und können beispielsweise kooperatives und problembasiertes Lernen anregen. ${ }^{122}$

Die Erstellung von WebQuests für den Arabischunterricht lohnt sich aus mehreren Gründen. Vorrangig, weil Schüler*innen vor eine authentische sprachliche Problematik gestellt werden, welche nur durch die Recherche und Benutzung authentischer Materialien gelöst werden kann. So können WebQuests auf spielerische und entdeckerische Weise sprachliche und kulturelle Kenntnisse vermitteln. Zudem kann die Lehrkraft die Schüler*innen gezielt in der Benutzung arabischsprachiger Internetseiten anleiten, in dem sie ausgesuchte Internetseiten in die WebQuests einbaut und durch Hyperlinks sicherstellt, dass die Schüler*innen sich nicht ziellos im Internet verirren, sondern nützliche und angebrachte Internetseiten besuchen und erforschen. Zudem ist der WebQuest an sich ein Bildungsmedium, welches sowohl authentische Inhalte präsentiert als auch andere Bildungsmedien wie Lehrbücher und Videos zur Bearbeitung der Aufgaben bereitstellt. Er kann dazu beitragen, die Schüler*innen in der sinnvollen, bedürfnisbezogenen Auswahl und Nutzung dieser Medien zu schulen, was laut der KMK eines der Hauptziele im Bereich Medienbildung ist. ${ }^{123}$ Letztlich kann durch die gezielte Auswahl an Links auch gewährleistet werden, dass Schüler*innen sich mit Lesetexten, Audio-

\footnotetext{
${ }^{121}$ Vgl. Lehrerinnenfortbildung Baden-Württemberg 2020.

${ }^{122}$ Vgl. Ständige Konferenz der Kultusminister der Länder in der Bundesrepublik Deutschland 2012, S. 4.

${ }^{123}$ Vgl. ebd., S. 5.
} 
material, Videos, Bildern und anderen Medien beschäftigen und so mehrere Arten von Input erhalten. ${ }^{124}$

\subsection{Multimediale Lernumgebungen}

\subsubsection{Individualisierte Internetseiten}

Auch Mike Turner stellt in seinem Bericht zum Einsatz neuer Medien an der University of Texas at Austin dar, wie die Erstellung einer Internetseite zur Verwirklichung der Unterrichtsziele beitragen kann. Er beschreibt einen Arabischkurs, in welchem modernes Hocharabisch und marokkanisches Arabisch gemeinsam unterrichtet werden. Ausgangsbasis für den Unterricht ist das Lehrwerk Al-Kitaab fii Ta'allum al- 'Arabiyya, Part One, 3rd ed ${ }^{125}$, welches Vokabeln, Aktivitäten und Texte auf Hocharabisch, levantinischem Arabisch und Ägyptisch-Arabisch enthält, sowie die dazugehörige Internetseite mit Hörtexten und Videos. ${ }^{126}$ Turner erläutert, dass der Ansatz des Lehrwerks auch für seinen Kurs nützlich ist, dass aber die Erweiterung des Lehrwerks selbst um marokkanisch-arabische Inhalte unmöglich sei und einer kompletten Überarbeitung bedürfe. Folglich wurde stattdessen eine neue Internetseite mit marokkanisch-arabischen Videos und Hörtexten entwickelt, welche den Stil der bereits existenten Internetseite nachahmt und fast alle behandelten Themenbereiche auch auf Marokkanisch zugänglich macht. Die Internetseite bietet vor allem den Vorteil, dass ein besonderes Augenmerk auf kulturelle Gepflogenheiten Marokkos und deren Auswirkungen auf Kommunikation gelegt werden kann, weil durch verschiedene Videos und Texte sowie Erklärungen zu kulturellen Aspekten ein gesamtheitlicheres Bild der sprachlichen Realität Marokkos geschaffen wird. ${ }^{127}$

Konkret heißt dies, dass die Internetseite interaktive Vokabellisten enthält, in denen jedes Wort mit einer Audiodatei verbunden ist. Marokkanische und hocharabische Worte werden nebeneinander präsentiert, wobei sich manchmal die Orthografie und die Aussprache eines bestimmten Wortes unterscheiden, manch-

\footnotetext{
${ }^{124}$ Vgl. Samy 2006, S. 272.

${ }^{125}$ Vgl. Brustad et al. 2015.

${ }^{126}$ Vgl. Georgetown University Press.

${ }^{127}$ Vgl. Turner 2018, S. 138.
} 
mal jedoch die Schreibweise gleichbleibt und lediglich die Aussprache abweicht. Vor allem hier kommen die integrierten Audiodateien den Lernenden zugute, durch welche sie die korrekte Aussprache so oft wie möglich anhören und gleichzeitig nachsprechen können. ${ }^{128}$ Darüber hinaus beinhaltet die Internetseite von Arabischlehrkräften erstellte Videos und Hörtexte, in denen die neuen Vokabeln verwendet werden. Die Videos wurden an Orten in Marokko gefilmt, um den Student*innen Einblicke in Architektur und Landschaft zu geben und Gesten und Gepflogenheiten bei Begrüßungen und Verabschiedungen zu veranschaulichen. ${ }^{129}$ Außerdem begrüßt der von Turner beschriebene Kurs ausdrücklich die Idee, dass kulturelles Wissen und interkulturelle Kompetenzen einen separaten Kompetenzbereich zu Sprechen, Hören, Schreiben und Lesen darstellen und entsprechend gefördert werden sollten. Zu diesem Zweck beinhaltet die Internetseite vielfältige kulturelle Informationen zu Themen wie Ehe und Familie, welche die Lernenden eigenständig lesen können, um sich kulturelles Wissen, welches möglicherweise im Unterricht selbst zu kurz kommt, anzueignen. Die Student*innen sollen ihr wachsendes Wissen zu angebrachten Begrüßungen, Ausrufen und Verabschiedungen regelmäßig auch im Unterricht einbringen. ${ }^{130}$

Die hier beschriebene Internetseite ist ein gutes Beispiel für den Anspruch der KMK, multimediale Lernumgebungen zu gestalten, in denen Schüler*innen eine Bandbreite von Input und Aktivitäten nutzen können. ${ }^{131}$ Sie verbindet inhaltliche Texte zur Kultur Marokkos mit Audiodateien, die zum Nachsprechen anregen und mit Videos, die zwar didaktisiert sind und von Lehrkräften erstellt wurden, jedoch versuchen, so viel Authentizität wie möglich zu schaffen. Wie Turner mehrmals betont, liegt der größte Vorteil der beschriebenen Internetseite darin, dass die Student*innen sich alle Audio- und Videodateien so oft wie möglich, zu jeder Zeit und an theoretisch jedem Ort anhören können. Dadurch können Lernunterschiede ausgeglichen werden und jeder Lernende kann sich, entsprechend seiner individuellen Bedürfnisse, auf die nächste Unterrichtseinheit vorbereiten. ${ }^{132}$ Auch die KMK betrachtet diesen Aspekt des Medieneinsatzes als besonders nutz-

\footnotetext{
128 Vgl. ebd., S. 142f.

${ }^{129}$ Vgl. ebd., S.149.

${ }^{130}$ Vgl. ebd., 2018, S. 149f.

${ }^{131}$ Vgl. Sekretariat der Kultusministerkonferenz 2016, S. 32.

132 Vgl. Turner 2018, S. 147.
} 
bringend, weil Bildungsmedien wie die beschriebene Internetseite dazu beitragen können, den persönlichen Kompetenzstand eines jeden zu berücksichtigen und individuell verfügbare Lösungen anzubieten. Dadurch können Lehrer*innen auch besser auf die Gegebenheiten in heterogenen Klassen reagieren. ${ }^{133}$ Zudem verfolgen die visuellen, interaktiven und informativen Hinweise auf die marokkanische Kultur das Ziel, kulturelles Verständnis auszubilden und Reflexionen seitens der Lernenden zu ihrer eigenen Kultur anzuregen. Dies ist einerseits wichtig, weil interkulturelle Kompetenzen ein grundlegender Bestandteil der fremdsprachlichen Ausbildung sind ${ }^{134}$ und andererseits, weil sie für Lernende einen essenziellen Aspekt des Arabischunterrichts darstellen können ${ }^{135}$, ohne welchen ihre Lernerfahrung unzufriedenstellend und lückenhaft wäre. ${ }^{136}$

Die beschriebene Internetseite wurde noch nicht offiziell in die Al-Kitaab-Webpräsenz von Georgetown University Press ${ }^{137}$ integriert und ist bisher nicht öffentlich verfügbar. Um eine Vorstellung von den Vokabellisten zu bekommen, kann man sich jedoch an den Beispielen in Al-Kitaab fii Ta'allum al-'Arabiyya, Part One, $3 r d e d^{138}$ orientieren. Auch die marokkanisch-arabischen Videos sind denen auf der Internetseite für Levantinisch und Ägyptisch sehr ähnlich. ${ }^{139}$

\subsubsection{Interaktive Videos}

Samy hebt den besonderen Nutzen von Videos, egal ob authentisch oder für den Unterricht entwickelt, für den Fremdsprachenunterricht hervor. Durch Videos können Lernende sehen, wie Muttersprachler*innen miteinander interagieren, wie sie Körperhaltung, Gesten und Mimik einsetzen und was dadurch ausgedrückt wird. Außerdem können Lernende ein Bewusstsein für suprasegmentale sprachliche Elemente bekommen, beispielsweise Geschwindigkeit, Rhythmus, Satzmelodie und Betonung. Statt diese Elemente nur im Unterricht zu besprechen oder sie sich beim Lesen eines Textes vorzustellen, zeigt ihnen ein Video einen Gesamt-

\footnotetext{
${ }^{133}$ Vgl. Sekretariat der Kultusministerkonferenz 2016, S. 32.

${ }^{134}$ Vgl. Sekretariat der Ständigen Konferenz der Kultusminister 2012, S. 2.

${ }^{135}$ Vgl. Behzadi et al. 2019, S. 421.

${ }^{136}$ Vgl. Zaki und Palmer 2018, S. 290.

${ }^{137}$ Vgl. Georgetown University Press.

${ }^{138}$ Vgl. Brustad et al. 2015.

${ }^{139}$ Vgl. Georgetown University Press.
} 
kontext, der alle Elemente auf natürliche Art und Weise vereinigt und den Lernenden hilft, sich diese wichtigen Aspekte der arabischen Sprache einzuprägen. ${ }^{140}$ Hinzu kommt die Tatsache, dass die hauptsächliche sprachliche Interaktion im Unterricht meist unter den Schüler*innen oder zwischen Schüler*innen und Lehrkraft stattfindet. Durch Videos können Lernende an die Stimmen und Ausdrucksweisen anderer Personen herangeführt werden, was sie auch auf ungeplante Interaktionen mit Muttersprachler*innen vorbereitet.

Eine Besonderheit der zuvor erwähnten Internetseite für Marokkanisch-Arabisch sind die interaktiven Videos, in welchen beispielsweise eine virtuelle Person Fragen stellt, worauf ein bestimmter Zeitraum folgt, in welchem die Lernenden darauf antworten können. Dadurch wird das Sprachenlernen in den eigenen vier Wänden etwas interaktiver gestaltet, als es durch reines Hören oder Lesen neuer Vokabeln und Texte der Fall wäre. ${ }^{141}$ Samy fügt dem hinzu, dass man solche virtuellen Interaktionen beispielsweise so gestalten kann, dass der oder die Lernende vorgegebene Antworten vorlesen soll, welche am Ende einen gesamten Dialog ergeben, den er oder sie sich so stückweise einprägen kann. Jeder Teil des Dialogs und der komplette Dialog in seiner Endform sollte aufgenommen werden können, sodass der oder die Lernende sich die gesamte Interaktion anhören und den eigenen Sprachgebrauch, vor allem in Bezug auf die Aussprache, reflektieren und bewerten kann. Dazu sollte auch ein Musterdialog bereitgestellt werden, der die angestrebte, korrekte Aussprache verdeutlicht. ${ }^{142}$ Der große Vorteil solcher virtuellen Interaktionen liegt darin, dass sie immer und immer wieder durchgeführt werden können und dass die Lernenden durch den virtuellen Gesprächspartner direktes und objektives Feedback erhalten. ${ }^{143}$ Einerseits kann so eine gewisse Angst, sich vor der eigenen Lehrkraft oder den Mitschüler*innen im Unterricht zu blamieren, verringert werden und andererseits können Lernende von einer anderen „Person“ als der Lehrkraft Rückmeldung erhalten, was zudem auch Zeit spart. Einfache, nach bestimmten Schemata verlaufende Konversationen können so regelmäßig geübt und bewertet werden und die Unterrichtszeit kann für komplexere Aktivitäten genutzt

\footnotetext{
${ }^{140}$ Vgl. Samy 2006, S. 264.

${ }^{141} \mathrm{Vgl}$. Turner 2018, S. 147.

${ }^{142}$ Vgl. Samy 2006, S. 268.

${ }^{143}$ Vgl. Sekretariat der Kultusministerkonferenz 2016, S. 32.
} 
werden, welche unbedingt Anleitung und Unterstützung durch eine reelle Person benötigen. ${ }^{144}$

Trotzdem sieht Samy in interaktiven Internetseiten auch einige Nachteile, welcher sich Lehrkräfte und Lernende bewusst sein müssen. Der Austausch mit virtuellen Muttersprachler*innen kann dazu führen, dass das Sprachenlernen zu einer individuellen Aktivität wird, die nicht mehr vorrangig im Klassenverbund oder einer anderen Art von Gemeinschaft stattfindet. Gemeinschaftliches Sprachenlernen ist jedoch essenziell, weil es die Schüler*innen anregt, sich auszutauschen, aufeinander zu reagieren und voneinander zu lernen. Sprachliche Aktivitäten finden in der Realität oft mit anderen Personen statt und sollten auch dementsprechend vermittelt werden. Spontan auf die Fragen und Meinungen anderer zu reagieren, trägt zudem zur Entwicklung sozialer Kompetenzen bei, die auch außerhalb des Klassenzimmers relevant sind. Folglich sollten interaktive Internetseiten zielgerichtet eingesetzt und den Bedürfnissen der Lernenden angepasst werden. Sie sollten nicht als Universallösung angesehen, sondern vielmehr als ein Unterrichtsmedium unter vielen betrachtet werden, welches für manche Situationen angebrachter ist als für andere. ${ }^{145}$

\subsection{Logistische Vorteile neuer Medien}

Wie bereits von Samy erwähnt, können digitale Hilfsmittel dazu beitragen, Zeit zu sparen und die tatsächliche Unterrichtseinheit besser zu nutzen. Brustad erläutert beispielsweise, dass das Vorlesen zu den wichtigen Fähigkeiten in der Sprachausbildung gehört, welche regelmäßig geübt werden sollte. Jedoch ist es unrealistisch, die Schüler*innen im Unterricht längere Texte vorlesen zu lassen, da dies, vor allem bei größeren Klassen, viel Zeit beansprucht. Sie schlägt vor, dass Arabischlernende, vor allem auf fortgeschritteneren Niveaus, das Vorlesen eigenständig zu Hause üben, sich dabei aufnehmen und die Aufnahme zur Kontrolle an die Lehrkraft schicken. ${ }^{146}$ Außerdem können Aufnahmen von anderen sprachlichen Situationen gemacht werden, zum Beispiel Diskussionen oder Unterhaltungen auf

\footnotetext{
${ }^{144}$ Vgl. Samy 2006, S. 268.

145 Vgl. ebd., S. 273.

146 Vgl. Brustad 2006, S. 342.
} 
Arabisch im Unterricht. Diese können die Lernenden sich dann immer wieder anhören, beispielsweise auf dem Weg vom oder zum Unterricht. So können sie sich die sprachlichen Mittel, die im Unterricht verwendet werden, immer wieder bewusst machen und sie eigenständig wiederholen. ${ }^{147}$ Auf diese Weise kann auch die Zeit außerhalb des Unterrichts für den Spracherwerb genutzt werden und die gemeinsame Unterrichtszeit kann für Aktivitäten verwendet werden, die Erklärungen und Anleitungen durch die Lehrkraft benötigen. Diese logistischen Vorteile, die neue Medien eröffnen, sind vor allem von Bedeutung, da die arabische Sprache als distante Sprache mehr Zeitaufwand benötigt und langsamere Lernfortschritte als andere Schulfremdsprachen erwarten lässt. ${ }^{148}$ Die tatsächliche Unterrichtszeit, in welcher der Kontakt zwischen Lehrenden und Lernenden gewährleistet ist, sollte so effektiv wie möglich genutzt und technische Hilfsmittel, die auch außerhalb des Unterrichts zum Lernfortschritt beitragen können, sollten aktiv eingesetzt werden.

\subsection{Erkenntnisse für den deutschen schulischen Kontext}

Wie bereits erwähnt, soll die Präsentation konkreter Anwendungsbereiche für digitale Medien im Arabischunterricht vor allem als Inspiration für Arabischlehrer*innen in Deutschland dienen. Ein essenzieller Aspekt, der hierbei jedoch bedacht werden muss, ist, dass sich deutsche Datenschutzrechte, vor allem im schulischen Kontext, deutlich von denen anderer Länder unterscheiden können und dass manche erwähnten Internetseiten möglicherweise nicht oder nur zum Teil in Deutschland verfügbar sind. Des Weiteren werden einige soziale Medien oder Kommunikationsprogramme nicht für den schulischen Unterricht geeignet sein. Darüber hinaus sind viele der hier genannten Anwendungen für Englischmuttersprachler*innen oder zumindest für den Arabischunterricht im englischsprachigen Kontext konzipiert. Man könnte viele Beispiele also nicht eins zu eins übernehmen, sondern müsste sie für den deutschsprachigen Kontext anpassen oder zumindest sicherstellen, dass die Arabischschüler*innen adäquate Englischkenntnisse besitzen.

\footnotetext{
${ }^{147}$ Vgl. Stevens 2006, S. 259.

${ }^{148}$ Vgl. Behzadi et al. 2019, S. 416.
} 
Dennoch zeigt sich, dass mehrere der oben beschriebenen Beispiele die Vorgaben der KMK bereits erfüllen würden oder zumindest gute Ausgangspunkte sind, um entsprechende Aktivitäten für den schulischen Arabischunterricht in Deutschland zu entwickeln. Vor allem sollte hier deutlich werden, dass neue Medien die Ausbildung aller Kompetenzbereiche unterstützen können und zudem wichtige kulturelle Einblicke in die arabische Welt gewähren. Ein besonderer Vorteil, der sich durch neue Kommunikationsmedien und Sofortnachrichtendienste bietet, ist der direkte und kostenfreie Austausch mit Arabischmuttersprachler*innen und die Tatsache, dass geografische Distanzen keine unüberwindbare Hürde für die Interaktion mit der arabischen Gesellschaft mehr darstellen. Zudem kann auch die außerunterrichtliche Lernzeit effizienter gestaltet werden, sodass Lernende ihren eigenen Bedürfnissen entsprechend auch ohne die Unterstützung der Lehrkraft an der Festigung ihrer Arabischkenntnisse arbeiten können. Zuletzt bieten digitale Medien verschiedenste Möglichkeiten für die Vermittlung dialektaler Kenntnisse und die Ausbildung von größerem Bewusstsein in Bezug auf die sprachlichen Varietäten des Arabischen. Jedoch sollte auch auffallen, dass, wie bereits in den Lehrplänen beobachtet, in den verschiedenen Unterrichtsbeispielen viel mehr Wert auf das Lernen mit digitalen Medien als auf das Lernen über sie gelegt wird. 


\section{Vergleich existenter (Bildungs-)medien}

Im folgenden Teil dieser Forschungsarbeit geht es darum, bereits existente digitale Medien für den Arabischunterricht zu vergleichen und hervorzuheben, wie diese zur Förderung bestimmter Kompetenzen beitragen können. Innerhalb dieses Vergleichs sollen die jeweiligen Stärken und Schwächen herausgearbeitet werden, um zu verdeutlichen, inwiefern digitale Medien die Unterrichtsgestaltung sinnvoll unterstützen oder aber behindern können.

Die KMK fordert von Lehrenden unter anderem, dass sie fähig sind, ,aus der Vielzahl der angebotenen Bildungsmedien (gewerbliche Angebote der Verlage und Open Educational Resources/OER) anhand entsprechender Qualitätskriterien für die Einzel- oder Gruppenarbeit geeignete Materialien und Programme zu

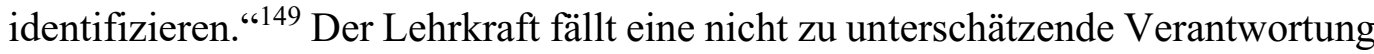
zu, denn idealerweise sollten die ausgewählten Medien datenschutzkonform und inhaltlich anspruchsvoll sein, den Interessen der Schüler*innen entsprechen und sowohl individualisierte als auch kollaborative Lernprozesse unterstützen. ${ }^{150}$ Bedenkt man die anderen Verantwortungen und Aufgaben, die eine Lehrkraft im schulischen Alltag zu übernehmen hat, wird schnell klar, dass diese Anforderungen die zeitliche Verfügbarkeit der Lehrkraft schnell übersteigen können. ${ }^{151}$ Die medienpädagogische Rolle der Lehrkraft kann nur adäquat ausgefüllt werden, wenn eine entsprechende Ausbildung in diesem Bereich gewährleistet wird, die passende Infrastruktur an der Schule existiert und die Unterstützung bundesweiter Akteure, wie beispielsweise dem Institut für Film und Bild in Wissenschaft oder dem Deutschen Bildungsserver, zur Verfügung steht. ${ }^{152}$ Wie in Kapitel 3 erwähnt, ist die Mehrzahl der Arabischlehrer*innen in Deutschland jedoch gewissermaßen auf sich allein gestellt und bisher existieren für sie keine formellen Aus- oder Weiterbildungsmöglichkeiten. Somit ist die Notwendigkeit für einen Rahmen oder eine Strategie für die Auswahl nützlicher, altersgerechter und inhaltlich anspruchsvoller Medien für den Arabischunterricht umso wichtiger. Insbesondere beim unreflektierten Einsatz von OER besteht die Gefahr, dass sie die Interessen be-

\footnotetext{
${ }^{149}$ Sekretariat der Kultusministerkonferenz 2016, S. 27.

${ }^{150}$ Vgl. ebd., S. 27.

${ }^{151}$ Vgl. Verbraucherzentrale Bundesverband 2016, S. 3.

152 Vgl. Sekretariat der Kultusministerkonferenz 2016, S. 34.
} 
stimmter wirtschaftlicher Akteure vertreten und die gesetzlich verankerte Neutralität von Schule beeinträchtigen. Hier werden Standards benötigt, anhand derer die Qualität verfügbarer OER für Unterrichtszwecke bewertet werden kann. ${ }^{153} \mathrm{Um}$ aus der Vielzahl arabischer Videos, Internetseiten, Lernspiele und anderer Materialien passend zu wählen, bedarf es genereller Kriterien für die Auswahl geeigneter Medien und zusätzlich bestimmte Kriterien, durch die der Nutzen eines Mediums für die Verwirklichung der Kompetenzziele des Arabischunterrichts definiert werden kann. Letztere existieren noch nicht. Generelle Kriterien für sinnstiftende Bildungsmedien im schulischen Kontext gibt es jedoch einige, unter anderem von der $\mathrm{KMK}^{154}$ und vom vzbv ${ }^{155}$ zusammengestellt.

\subsection{Die Kriterien}

Laut den Qualitätskriterien für Bildungsmedien der KMK sollten gute Bildungsmedien inhaltlich korrekt und lehrplankonform sein und den kompetenzorientierten Unterricht sowie individuelle Lernprozesse unterstützen. Weiterhin gelten Multimedialität, Interaktivität, Vernetzbarkeit, Veränderbarkeit und Teilbarkeit als Aspekte sinnstiftender Bildungsmedien. ${ }^{156}$ Die Bewertungskriterien für Unterrichtsmaterial des vzbv sind in die Bereiche „Fachlicher Inhalt", „Methodik und Didaktik“ und „Formale Gestaltung“ aufgeteilt. Das Kriterium „Fachlicher Inhalt“ bewertet beispielsweise, ob das Medium Inhalte plural und multiperspektivisch darstellt und den Lerngegenstand im gesellschaftlichen Kontext zeigt. Außerdem sollten Inhalte sachrichtig und unparteiisch gestaltet sein und weder Werbung noch andere tendenziöse Darstellungen enthalten. Unter „Methodik und Didaktik“ fallen Kriterien wie die moderne und zeitgemäße Vermittlung von Inhalten und die Aufnahme der Interessen und Erfahrungen von Schüler*innen. Darüber hinaus sollen Medien Bildungs- und Lernziele klar formulieren und methodisch abwechslungsreich sein, vielseitige Arbeitsaufträge ermöglichen und, wenn möglich, didaktische Begleittexte beinhalten. Zuletzt sollten Medien für den schulischen Unterricht laut dem Kriterium „Formale Gestaltung“ eine gute inhaltliche

\footnotetext{
${ }^{153}$ Vgl. Verbraucherzentrale Bundesverband 2016, S. 9.

${ }^{154}$ Vgl. Sekretariat der Kultusministerkonferenz 2016, S. 33.

${ }^{155}$ Vgl. Verbraucherzentrale Bundesverband.

${ }^{156}$ Vgl. Sekretariat der Kultusministerkonferenz 2016, S. 33.
} 
Strukturierung und Gliederung aufweisen und zielgruppengerecht grafisch gestaltet sein, um den Stoff verständlicher zu vermitteln. ${ }^{157}$

Im Folgenden soll auf verschiedene mediale Lernangebote eingegangen und die Vor- und Nachteile ihrer Verwendung sollen herausgearbeitet werden. Hierbei werden sowohl weniger geeignete als auch vielversprechende Lernangebote in Betracht gezogen, um zu verdeutlichen, wo potenzielle Defizite und Vorteile bei Online-Ressourcen liegen können. Da der Forschungsstand im Bereich AraF in Deutschland gering und schulischer Arabischunterricht in Deutschland kaum vorzufinden ist, sind alle beschriebenen Internetseiten und Anwendungen ursprünglich für englischsprachige Lernende konzipiert. Dies ist bei einigen Angeboten aber nicht unbedingt problematisch und sie können trotzdem zur Unterrichtsgestaltung im deutschsprachigen Kontext beitragen.

\subsection{Duolingo ${ }^{158}$}

Duolingo ist eine seit 2012 frei verfügbare Lernapp, die laut eigener Aussage die beste neue Methode ist, eine Sprache zu lernen. ${ }^{159}$ Duolingo ist grundsätzlich kostenfrei und von jedem Smartphone aus benutzbar. ${ }^{160}$ Die Mission dieser Lernapp ist es, ,allen ein persönliches Lernerlebnis mittels Technologie zu ermöglichen.“161 Obwohl Duolingo bewusst versucht, viele Aspekte des Sprachenlernens spielerisch zu gestalten, betont die Internetseite, dass das Design der App dennoch auf einer Methodik basiert, ,die nachweislich das Langzeitgedächtnis fördert, und auf einem Lehrplan, der an einem internationalen Standard ausgerichtet ist. “162 Die Methodik wird auf der offiziellen Internetseite etwas genauer beschrieben und soll einen funktionalen Ansatz verfolgen, der Lernende dazu befähigt, in realen kommunikativen Situationen, beispielsweise im Restaurant, erfolgreich zu agieren. Des Weiteren sollen laut Duolingo die vier Kompetenzen Lesen, Schreiben, Hören und Sprechen geschult werden. Außerdem sollen „Sprachregeln“ und Muster vom

\footnotetext{
${ }^{157}$ Vgl. Verbraucherzentrale Bundesverband.

${ }^{158}$ Duolingo ist als mobile Anwendungssoftware verfügbar unter https://apps.apple.com/de/app/duolingo/id570060128

${ }^{159}$ Vgl. Duolingo (b).

${ }^{160}$ Vgl. ebd.

${ }^{161}$ Vgl. Duolingo (d).

162 Duolingo (c).
} 
Lernenden selbst entdeckt werden und somit den Ansatz des implizierten Lernens unterstützen. ${ }^{163}$ Die internationalen Standards, an denen sich die Lektionen in der App orientieren, werden jedoch nicht näher ausgeführt.

Manche Vorteile, die man Duolingo zuschreiben könnte, sind die grafisch ansprechende Darstellung der Inhalte und das Schaffen von Abwechslung innerhalb der App durch Animationen und Bilder. Zudem unterstützt die Verfügbarkeit der App auf Smartphones die Möglichkeit, auch außerhalb des Unterrichts und unabhängig von Ort und Zeit zu lernen. Darüber hinaus sind die Inhalte, welche sich vorrangig aus Vokabeln zusammensetzen, in verschiedene Kategorien wie Geografie, Familie, Kleidung, Essen und Hobbies gegliedert, wodurch die Orientierung innerhalb der App erleichtert und zur zielgerichteten Festigung bestimmter Vokabeln beigetragen werden kann. Hinzu kommt die Tatsache, dass Duolingo anstrebt, Lernenden individuelles Lernmaterial mit dem richtigen Schwierigkeitsgrad bereitzustellen. Dieser Prozess findet durch bestimmte Algorithmen innerhalb der App statt, die das Lernverhalten der Lernenden beobachten und den Schwierigkeitsgrad der Übungen entsprechend anpassen. ${ }^{164}$ Theoretisch könnte dies dazu beitragen, Lernenden personalisiertes Feedback zu geben und sie individueller zu fördern.

Duolingo wird weltweit von über 300 Millionen Menschen benutzt und erfreut sich, zumindest laut eigener Aussage, großer Beliebtheit bei Lehrer*innen, Schüler*innen und sogar Regierungen. ${ }^{165}$ Für den deutschen schulischen Kontext ist jedoch festzustellen, dass Duolingo kaum von Nutzen sein wird. Die erste Hürde ist, dass, obwohl einige Sprachen für Deutschmuttersprachler*innen verfügbar sind, die Arabischlektionen ausschließlich mit englischer Übersetzung existieren. Dies setzt also ein adäquates Englischniveau unter den Schüler*innen voraus. Hinzu kommt die Tatsache, dass die App mit Lektionen zum Wiedererkennen und Aussprechen der arabischen Schriftzeichen beginnt. Hierbei gibt es jedoch keine Anleitung $\mathrm{zu}$ den verschiedenen Formen, welche die arabischen Buchstaben innerhalb eines Wortes annehmen, je nachdem ob sie zu Beginn, in der Mitte oder am Endes des Wortes stehen. Es wird auch nicht näher darauf eingegangen, welche Buchstaben sich rechts- und linksbündig verbinden und welche nicht. Zudem

\footnotetext{
${ }^{163}$ Vgl. Duolingo (c).

164 Vgl. ebd.

165 Vgl. Duolingo (a).
} 
bemerkt man bereits nach einigen Übungen, dass vor allem die emphatischen Laute in den Einführungslektionen anders ausgesprochen werden als dies in ganzen Worten in den späteren Lektionen der Fall ist. Darüber hinaus ist nicht nachvollziehbar, wie Duolingo Sprechkompetenz ausbilden soll. Hier könnte möglicherweise das individuelle Nachsprechen der vorgegebenen Vokabeln gemeint sein. Zuletzt ist auch anzumerken, dass die eigentliche Schreibkompetenz auf keine Weise gefördert wird, da Wörter nie selbst aus Buchstaben zusammengesetzt werden müssen und lediglich in ihrer digital dargestellten Form vermittelt werden, die Lernenden also nie selbst auf Arabisch schreiben.

Überdies ist zu beobachten, dass die Funktion einzelner Buchstaben wie des $t \bar{a}^{\prime}$ marbūta nicht näher erklärt wird und deshalb beispielsweise ohne Vorkenntnisse nicht nachzuvollziehen ist, warum und wann der Buchstabe $t \bar{a}^{\prime}$ marbūta seine Form ändert. Außerdem wird das $t \bar{a}^{\prime}$ marbūṭa innerhalb von Genitivverbindungen, welche als grammatisches Konstrukt an sich auch nicht näher erklärt werden, konsequent falsch ausgesprochen. Dies ist lediglich ein Beispiel für die vielen Aussprachefehler, die innerhalb der App auftreten. Auch die Aussprache des bestimmten Artikels al nach der Präposition $f i$ (,,in“) ist fehlerhaft und teilweise werden die langen Vokale $\bar{l}$ und $\bar{u}$ als Diphthonge ausgesprochen, wo dies eigentlich nicht der Fall ist. Ein weiterer Kritikpunkt ist, dass dialektale und hocharabische Elemente teils frei gemischt werden, ohne dass zuvor erklärt wurde, dass Unterschiede zwischen der Hoch- und Umgangssprache im Arabischen existieren. Obwohl dieser Aspekt die alltägliche Kommunikation nicht unbedingt behindert, wäre es zumindest hilfreich, Lernenden grundlegende Kenntnisse über die sprachlichen Varietäten des Arabischen zu vermitteln.

Die erwähnten Ungereimtheiten innerhalb der App lassen die finale Einschätzung zu, dass Duolingo als Anwendung dem tatsächlichen Lernprozess eher ab- als zuträglich ist und unter Lernenden wahrscheinlich mehr Verwirrung als sprachliches Wissen schafft. Sollte die Lehrkraft jedoch versuchen wollen, Duolingo in den Unterricht einzubeziehen oder die Schüler*innen zur eigenständigen Verwendung der App außerhalb des Unterrichts anzuhalten, müssen im Vor- und Nachhinein intensive Erklärungen zu Orthografie, Ausspracheregeln, wichtigen grammatischen Konzepten und den Varietäten der arabischen Sprache folgen. Duolingo kann keinesfalls angewendet werden, um diese Konzepte zu festigen oder ein- 
zuüben, weil die App korrektem Sprachgebrauch tatsächlich eher entgegenwirkt, als ihn zu fördern. Der einzig denkbare Nutzen der App Duolingo ist die Wiederholung von Vokabeln in verschiedenen alltäglichen Bereichen, welche möglicherweise durch die Verwendung in verschiedenen Sätzen ${ }^{166}$ oder in Verbindung mit Illustrationen in der App leichter im Gedächtnis bleiben können. ${ }^{167}$ Zudem besteht die Option, sich mit anderen Lernenden auf Duolingo auszutauschen und gegeneinander anzutreten. Diese Wettbewerbsfunktion könnte auf Schüler*innen motivierend wirken und zur langfristigen Festigung der Vokabeln beitragen. ${ }^{168}$ Abschließend ist jedoch auch anzumerken, dass innerhalb der App regelmäßig Werbeanzeigen verschiedener Anbieter auftreten. Somit ist Duolingo als Lernapp weder sachrichtig noch neutral. Die Zeit, die erforderlich ist, um Schüler*innen im sinnstiftenden Gebrauch der App anzuleiten und die Unstimmigkeiten innerhalb der App zu erklären, lohnt sich keinesfalls, wenn man den geringen Nutzen bedenkt, den die Übungen in der App erzielen können.

\subsection{InterLingo ${ }^{169}$}

InterLingo ist eine britische Internetseite, die sich vor allem an Lehrkräfte des Arabischen an britischen Schulen richtet. ${ }^{170}$ Obwohl InterLingo grundsätzlich für Lehrkräfte und Schüler*innen im englischsprachigen Raum konzipiert ist, kann sie trotzdem auch für Arabischlehrer*innen in Deutschland sinnvolle Anregungen und Materialien bieten, da sich die Inhalte und Übungen speziell an Schüler*innen der Sekundarschulen richten. Entsprechend sind einige Themen bereits altersgemäß aufbereitet und oft bedarf es lediglich weniger Anpassungen der Übungen, um die Inhalte und Arbeitsblätter auch für deutschsprachige Schüler*innen mit geringeren Englischkenntnissen bereitzustellen.

InterLingo zeichnet sich als Internetseite dadurch aus, dass Inhalte und Übungen zum einen nach Kompetenzbereichen - Schreiben, Lesen, Hören und Sprechen sortiert sind und zum anderen ein zusätzlicher Bereich für Grammatik verfügbar

\footnotetext{
166 Vgl. Baicheng 2009, S. 183.

${ }^{167}$ Vgl. Nikolova 2002, S. 115.

${ }^{168}$ Vgl. Wei et al. 2018, S. 156.

${ }^{169}$ InterLingo ist verfügbar unter https://www.interlingo.co.uk/

${ }^{170}$ Vgl. InterLingo Languages 2020b.
} 
ist. Außerdem sind die jeweiligen Kompetenzbereiche in drei Niveaustufen Beginner, Intermediate und Advanced - unterteilt. Dies erleichtert die Auswahl passender Übungen für die Lehrkraft und die Schüler*innen. Die komplette Nutzung der Internetseite verlangt eine Registrierung und einen Mitgliedsbeitrag; einige Ressourcen sind aber auch frei zugänglich und kostenlos nutzbar. Der Bereich Sample Resources bietet eine Auswahl an kostenlosen Übungen, die das Prinzip von InterLingo veranschaulichen: Hier gibt es beispielsweise Übungen, bei denen die Schüler*innen Bilder auf Arabisch beschreiben sollen. Die Beschreibung kann direkt auf der Internetseite als Audiodatei aufgenommen und heruntergeladen werden, sodass diese dann von der Lehrkraft kontrolliert werden kann. Andere Übungen beinhalten für den Unterricht konzipierte Hörtexte, beispielsweise eine kurze Beschreibung einer Person und eine dazugehörige MultipleChoice-Aufgabe. Darauf folgt eine Übung, bei der die arabischen Vokabeln ihren englischen Entsprechungen hinzugefügt werden müssen. Im Bereich für fortgeschrittene Lernende gibt es auch Hörübungen, bei denen sich die Aufgaben auf authentische Videos beziehen, beispielsweise von Sendern wie Al Jazeera, und im Anschluss Lückentexte ausgefüllt oder Verständnisfragen beantwortet werden müssen. Die meisten Höraufgaben umfassen nur hocharabische Inhalte und auch die Übungen in den anderen Kompetenzbereichen sind vorrangig auf Hocharabisch verfasst. Ein großer Vorteil der Höraufgaben ist, dass sie in der Regel ein vollständiges Transkript beinhalten.

Auf der Internetseite finden sich auch einige Lese- und Schreibübungen, bei denen beispielsweise Lückentexte mit passenden Vokabeln vervollständigt oder eigene Antworten zu bestimmten Fragestellungen verfasst werden sollen. Der Vorteil dieser Übungen ist, dass sie entweder direkt online ausgeführt werden können oder zum Ausdrucken bereitstehen. So kann je nach Bedürfnissen der Schüler*innen das Schreiben mit der Hand oder auf dem Computer geübt werden. Bei den fortgeschritteneren Aufgaben gibt es auch keine englischsprachigen Fragen oder Aufgabenstellungen mehr, was bedeutet, dass hierfür keine Englischkenntnisse vonnöten sind und die Aufgaben für deutsche Schüler*innen auf dem gleichen Arabischniveau übernommen werden können.

InterLingo ist eine gut strukturierte Internetseite, auf welcher Aufgaben in verschiedenen Kompetenzbereichen und für verschiedene Lernniveaus schnell 
gefunden werden können. Die angebotenen Übungen und Arbeitsblätter unterscheiden sich inhaltlich stark und beinhalten oft verschiedene Aktivitäten, was einen gewissen Grad an Abwechslung garantiert. Die Übungen sind leicht mit anderen Lehrkräften oder Schüler*innen zu teilen und können, auch wenn sie sich ursprünglich an ein englischsprachiges Publikum richten, für den deutschsprachigen Kontext verändert und angepasst werden. Dies setzt jedoch entsprechende Englischkenntnisse der Lehrkraft voraus. Den fachlichen Inhalt betreffend, fällt es der Lehrkraft zu, die Arbeitsblätter und Übungen genauestens zu kontrollieren und die sprachliche Richtigkeit zu bestätigen. Die authentischen Videos und Hörtexte zeichnen sich insofern durch Sachrichtigkeit und Neutralität aus, als dass sie unverändert als Teil der Aufgabe präsentiert werden. Jedoch behandeln manche Übungen Themen wie beispielsweise den palästinensischen Tag des Bodens, welche zusätzlicher Erklärungen zu geschichtlichen und politischen Geschehnissen bedürfen und gegebenenfalls erheblich modifiziert werden müssen. Werbung findet sich auf InterLingo keine. Die Übungen an sich verlangen durch die verschiedenen Aufgabenstellungen vielseitige Arbeitsaufträge, welche aber durchaus von der Lehrkraft weiter ausgebaut und anderweitig kreativ umgesetzt werden können.

\subsection{Defense Language Institute Foreign Language Center eLearning $^{171}$}

Das Defense Language Institute (DLI) ist eine Bildungs- und Forschungseinrichtung des US-Verteidigungsministeriums, die aus zwei Abteilungen besteht, welche Mitarbeiter*innen des Verteidigungsministeriums und anderer Bundesbehörden, vor allem Mitgliedern des US-Militärs, Fremdsprachenunterricht erteilen. Eine der beiden Einrichtungen ist das Defense Language Institute Foreign Language Center (DLIFLC), welches seinen Sitz im Bundesstaat Kalifornien hat und Unterricht in über 12 Sprachen anbietet. Es gilt als eine der renommiertesten Institutionen im Bereich der fremdsprachlichen Ausbildung in den USA und

${ }^{171}$ Das eLearning-Portal ist verfügbar unter https://www.dliflc.edu/online-learning/ 
zeichnet sich durch Ganztagsunterricht an fünf Tagen in der Woche sowie fakultative immersion programs aus. ${ }^{172}$

Die offizielle Internetseite des DLIFLC bietet eine Fülle an eLearning-Materialien für Arabisch, welche nach aktuellem Stand frei verfügbar und in den meisten Fällen kostenlos downloadbar sind. Die Datenbank an Texten und Übungen ist sehr groß und wäre für Schüler*innen vermutlich relativ undurchsichtig. Für Arabischlehrkräfte kann sie jedoch einige qualitativ hochwertige Materialien zur Unterstützung des Unterrichts anbieten. Da die verfügbaren Materialien vor allem in der Fremdsprachenausbildung des Militärs verwendet werden, sind viele Texte und Übungen inhaltlich nicht relevant für den schulischen Kontext. Die Benutzung dieser Datenbank erfordert in jedem Falle eine kritische Auswahl seitens der Lehrkraft und eine genaue Prüfung der Inhalte, obwohl dies theoretisch bei jeder Suche nach Unterrichtsmaterialien im Internet vonnöten ist. Im Folgenden sollen die wichtigsten Elemente des eLearning-Portals des DLIFLC kurz zusammengefasst werden.

\subsubsection{Arabic Phone Conversations ${ }^{173}$}

Diese Datenbank beinhaltet, wie der Name vermuten lässt, über 300 Telefongespräche auf Arabisch. Hierbei kann zwischen Gesprächen auf Ägyptisch, Irakisch oder Levantinisch gewählt werden, da die Telefongespräche vor allem dazu beitragen sollen, Schüler*innen mit den Dialekten der arabischen Welt vertraut zu machen. Die Gespräche sind nach Sprachniveaus unterteilt und können aus verschiedenen Themenbereichen wie beispielsweise Bildung, Einkaufen oder Essen und Trinken gewählt werden. Zu jeder Audiodatei gibt es Arbeitsblätter auf Englisch und Arabisch, mit der Möglichkeit, diese entweder unverändert oder als Muster für neue Unterrichtsaktivitäten zu übernehmen. Die verfügbaren Gespräche sind nicht für Anfänger geeignet, sondern erfordern gute Vorkenntnisse der Dialekte.

\footnotetext{
${ }^{172}$ Vgl. DLIFLC - Defense Language Institute Foreign Language Center.

${ }^{173}$ Die Arabic Phone Conversations sind verfügbar unter https://phone.dliflc.edu/default_arabic.asp
} 


\subsubsection{Arabic Accents Library ${ }^{174}$}

Diese elektronische Bibliothek umfasst eine Sammlung von Audiodateien, welche die sprachlichen Varietäten des Arabischen darstellen. Die Hörtexte sind auch hier nach verschiedenen Niveaus und Themenbereichen sortiert und setzen solide Vorkenntnisse voraus. Die verfügbaren Themen sind ausgesprochen vielfältig und beinhalten Texte zu Architektur, Verkehr, Bildung, Hochzeiten, arabischen Städten und vielen anderen Bereichen. Dazu gehören unter anderem Militär, Politik und Sicherheit. Die Inhalte müssen von der Lehrkraft kritisch und zielgerichtet ausgesucht werden und gegebenenfalls notwendiges Hintergrundwissen muss entsprechend vermittelt werden, um diese Hörtexte in einen größeren Kontext einzubetten. Der Vorteil dieser Sammlung ist, dass jeder Hörtext sowohl auf Hocharabisch als auch in zehn weiteren arabischen Dialekten verfügbar ist. Der Inhalt und der Aufbau des Textes sind gleich; lediglich die Vokabeln und die Aussprache unterscheiden sich je nach Dialekt, weshalb diese Dateien sich vor allem für sprachliche Vergleiche anbieten und beispielsweise in der Vermittlung dialektaler Kenntnisse auf Basis des Hocharabischen nützen können. Alle Dateien können heruntergeladen und geteilt werden.

\subsubsection{Language Survival Kits ${ }^{175}$}

Die Language Survival Kits sind eigentlich als Ressource für das Militär gedacht und beinhalten Vokabeln und Sätze sowie dazugehörige Audioaufnahmen für bestimmte Einsatzbereiche. Einige dieser Einsatzbereiche sind für den schulischen Fremdsprachenunterricht irrelevant, weil sie sich beispielsweise mit Marinekommandos und Waffen beschäftigen. Es gibt jedoch auch Bereiche wie ,grundlegende Sprache“, welche durchaus nützliches Vokabular und entsprechende Audioaufnahmen umfassen. Die Inhalte sind ausschließlich in Dialekten verfügbar, wobei aus zwölf Dialekten gewählt werden kann. Entscheidet man sich beispielsweise für Jordanisch-Arabisch und ,grundlegende Sprache“, erfolgt eine Weiterleitung auf eine Seite mit genaueren Möglichkeiten zur Eingrenzung des Themen-

\footnotetext{
${ }^{174}$ Die Arabic Accents Library ist verfügbar unter https://accents.dliflc.edu/

${ }^{175}$ Die Language Survival Kits sind verfügbar unter https://fieldsupport.dliflc.edu/productList.aspx? $=1$ sk
} 
bereichs, unter anderem mit Fokus auf Richtungsangaben, Wochentage, Familie und Wetter. Anschließend erscheint eine Liste relevanter Vokabeln, welche auf Arabisch, Englisch und in Umschrift ausgeschrieben sind und deren Aussprache man sich anhören kann. Sowohl die Vokabellisten als auch die Audiodateien stehen zum Download zur Verfügung. Diese Vokabellisten richten sich speziell an Anfänger und könnten, Kenntnis der arabischen Schriftzeichen vorausgesetzt, von Lernenden eines jeden Sprachniveaus genutzt werden.

\subsubsection{Global Language Online Support System (GLOSS) $)^{176}$}

GLOSS ist eine Datenbank von Online-Übungen in den Bereichen Hör- und Leseverstehen, welche sich vorrangig an fortgeschrittene und selbstständige Lernende richten. Es sind jedoch auch einige Übungen auf dem Anfängerniveau verfügbar. Auf GLOSS können Aufgaben auf Hocharabisch und in sechs weiteren Dialekten gewählt werden. Die Übungen können nach Niveaustufe, Kompetenzbereich - Lesen oder Hören - und Themenbereich ausgesucht werden. Typische Aufgaben beinhalten entweder einen Lese- oder Hörtext, Vokabelfragen, Verständnisfragen auf Englisch, Lückentexte und ein abschließendes Quiz. Da die Aufgabenstellungen innerhalb von GLOSS oft auf Englisch sind und auch viele der Verständnisfragen auf Englisch beantwortet werden müssen, setzt die Verwendung dieser Datenbank gute bis sehr gute Englischkenntnisse unter den Schüler*innen voraus. Zudem gibt es fehlerhafte Audiodateien, die nicht den verfügbaren Transkripten entsprechen, weshalb auch hier die Inhalte vorerst von der Lehrkraft geprüft und etwaige Fehler erkannt werden müssen. GLOSS ist leicht zu navigieren, kann aber durch die Fülle an Inhalten schnell überfordern. Für die eigenständige Verwendung seitens der Schüler*innen kann diese Internetseite nicht empfohlen werden. Für Lehrkräfte können die verfügbaren Aufgaben jedoch Anregungen zur Gestaltung eigener Übungen bieten.

Bei der Nutzung der Ressourcen des DLIFLC muss bedacht werden, dass die verfügbaren Materialien vorrangig der Ausbildung des Militärs dienen und oftmals für den schulischen Arabischunterricht irrelevant sind. Die Lehrkraft sollte hier

\footnotetext{
${ }^{176}$ GLOSS ist verfügbar unter https://gloss.dliflc.edu/
} 
kritisch und bedürfnisbezogen wählen. Grundsätzlich unterstützen die verfügbaren Materialien nur die Festigung der Lese- und Hörkompetenz und bieten keine Gelegenheiten für interaktive Sprechübungen oder den Ausbau schriftlicher Kompetenzen. Die Datenbanken sind insofern multimedial angelegt, als dass Hörund Lesetexte sowie vereinzelte Videos bereitgestellt werden. Der große Vorteil dieser Internetseite ist, dass fast alle Dateien heruntergeladen, geteilt und weiterverarbeitet werden können. Tatsächlich bietet das sehr weit gefasste Themenspektrum einen hohen Grad an Abwechslung hinsichtlich der vermittelten Inhalte. Die Datenbanken sind gut strukturiert und gegliedert, beinhalten aber keine grafischen Darstellungen, die bei der Vermittlung neuer Inhalte helfen könnten. Die Zuhilfenahme der Ressourcen des DLIFLC bietet sich vor allem für das Unterrichten der arabischen Dialekte und für sprachliche Vergleiche der Varietäten des Arabischen an. Eine große Hürde in der Benutzung der Datenbanken des DLIFLC ist, dass sie gute bis sehr gute Englischkenntnisse auf Seiten der Lehrkraft voraussetzt, um aus den angebotenen Materialien zu wählen und auf Seiten der Schüler*innen, um Online-Übungen selbstständig zu bearbeiten.

\subsection{Lingualism ${ }^{177}$}

Die Internetseite Lingualism versucht vorrangig dazu beizutragen, mehr Materialien für das Erlernen der arabischen Dialekte bereitzustellen und widmet sich dementsprechend vor allem Hörtexten in den verschiedenen Varietäten des Arabischen. ${ }^{178}$ Gleichwohl sind auch Inhalte auf Hocharabisch verfügbar. Lingualism ist nicht ausschließlich online zu finden, sondern funktioniert hauptsächlich in Verbindung mit den Lingualism-Lehrbüchern. Eines davon ist Arabic Voices, welches aus zwei Teilen besteht. Arabic Voices $1^{179}$ umfasst 28 Audio-Essays zu einer Vielzahl von Themen, jeder von einem Muttersprachler oder einer Muttersprachlerin gesprochen. Das Buch enthält Hörtexte auf Hocharabisch und den Dialekten Ägyptens, Tunesiens, Marokkos, Jordaniens, Palästinas, des Libanon, des Irak und des Jemen. $\mathrm{Zu}$ jedem Hörtext gehören verschiedene Aufgaben zum Hörverstehen auf Englisch und Arabisch, ein vokalisiertes Transkript mit englischer Übersetzung

\footnotetext{
${ }^{177}$ Die Lingualism-Internetseite ist verfügbar unter https://lingualism.com/\#languages ${ }^{178}$ Vgl. Lingualism 2020b.

${ }^{179}$ Vgl. Aldrich 2014.
} 
und wichtige Anmerkungen zu bestimmten Ausdrücken. Zudem folgt oft eine Erklärung der wichtigsten grammatischen Konzepte, die in einem Hörtext vorkommen. Im ersten Kapitel beispielsweise wird ein Hörtext auf Hocharabisch behandelt und die Verwendung des Konjunktivs anhand konkreter Beispiele aus dem Text erklärt. Jedes Kapitel beinhaltet mehrere Aufgabenstellungen, obwohl sich die Formate der Übungen innerhalb des Buches wiederholen. Die Themenbereiche sind jedoch sehr vielfältig und reichen von Kleidung, öffentlichen Verkehrsmitteln, Heirat und Dating bis zu den Revolutionen der arabischen Länder und den arabischen Königsfamilien.

Idealerweise sollten die von Lingualism bereitgestellten Hörtexte in Verbindung mit dem jeweiligen Lehrbuch im Unterricht verwendet werden, um einen maximalen Nutzen zu erzielen. Trotzdem ist nicht außer Acht zu lassen, dass Lingualism alle Hörtexte aus allen Lehrbüchern kostenlos auf der Internetseite und auch auf dem gleichnamigen YouTube-Kanal ${ }^{180}$ zur Verfügung stellt. Dies allein bietet einen großen Bestand an authentischem Audiomaterial, welches zur Vermittlung hocharabischer und dialektaler Kenntnisse genutzt werden kann. Dieser Bestand umfasst unter anderem die bereits erwähnten Hörtexte aus Arabic Voices, die Egyptian Arabic Diaries, eine Libanesisch-Arabische Serie namens Haki Kill Yoom, die Aussprache verschiedener Verben auf Ägyptisch-Arabisch und Levantinisch-Arabisch sowie Alltagsvokabeln in den Dialekten Tunesiens und Marokkos. Hinzu kommt eine Reihe namens Syrian Arabic Voices mit alleinigem Fokus auf die verschiedenen Varietäten Syriens und das Kinderbuch Mido, welches auf Hocharabisch, Levantinisch, Ägyptisch und Tunesisch zur Verfügung steht.

Die Internetseite und der YouTube-Kanal sind gut strukturiert, da alle Audiodateien nach Lehrbüchern bzw. nach Sprachvarietät sortiert sind. Zudem stehen alle Audiomaterialien auch zum Download bereit. Die Lingualism-Internetseite verwendet keine Werbung, ist aber kommerziell gestaltet und verlinkt an vielen Stellen auf den Lingualism-Shop. Durch die Verwendung von Hörmaterialien, Lehrbüchern und Videos der Lingualism-Serie kann ein hoher Grad an Multimedialität geschaffen werden. Lingualisms Produkte können hauptsächlich zur Ausbildung besseren Hörverstehens beitragen und wichtige Einblicke in die

\footnotetext{
${ }^{180}$ Der YouTube-Kanal ist verfügbar unter https://www.youtube.com/user/lingualism
} 
arabische Kultur gewähren. Die Texte selbst sind insofern unparteiisch, als dass Muttersprachler*innen ihre Gedanken und Meinungen frei ausdrücken. Hier muss jedoch bedacht werden, dass bestimmte Themenbereiche und Fragen vorgegeben wurden, um die Hörtexte zu produzieren. Dennoch schafft Lingualism es, Themen auch multiperspektivisch darzustellen. Das Thema Heirat beispielsweise wird von verschiedenen Personen aus verschiedenen Ländern besprochen. Was Lingualism jedoch grundsätzlich fehlt, sind Hintergrundinformationen $\mathrm{zu}$ den sprachlichen Varietäten der arabischen Welt. Die sprachlichen Inhalte werden neutral dargestellt, aber die Entstehung einzelner Ausdrücke, deren Verwendung in bestimmten soziokulturellen Kontexten oder die Beziehungen zwischen den Dialekten und Hocharabisch werden nicht thematisiert. Hier kommt es der Lehrkraft zu, Schüler*innen adäquates Hintergrundwissen zu vermitteln und die einzelnen Hörtexte in einen größeren Kontext einzubetten. Zudem ist zu beachten, dass sich Lingualism vorrangig an fortgeschrittenere Schüler*innen auf den Niveaus B1/B2 laut Europäischem Referenzrahmen ${ }^{181}$ und darüber richtet.

Lingualism bietet jedoch eine zweite wichtige Dimension des Arabischlernens, und zwar die größere Lingualism-Gemeinschaft. Auf der Internetseite kann man ein kostenloses Profil erstellen, welches einem dann erlaubt, mehreren Gruppen beizutreten und sich an den Diskussionen in den Foren zu beteiligen, wobei die Gruppen nach Sprachvarietäten unterteilt sind. Auf den Foren können Fragen zu verschiedensten Aspekten der arabischen Sprache gestellt werden, worauf andere Mitglieder und Mitarbeiter*innen des Lingualism-Teams antworten können. So kann ein freier Austausch unter Arabischlernenden entstehen, der es Schüler*innen erlaubt, Fragen zu stellen, die im Unterricht zur kurz gekommen sind oder grundsätzlich von den Erfahrungen anderer Arabischlernender zu profitieren. Jede Gruppe eröffnet zudem weitere Möglichkeiten für die selbstständige Vertiefung der Arabischkenntnisse. In der Levantinisch-Arabisch-Gruppe beispielsweise finden sich Links zu Musikvideos mit kompletten Transkripten und zusätzlicher englischer Übersetzung. Außerdem hat man Zugriff auf Vokabellisten zu verschiedenen Themen wie Körperteile und Adjektive, welche Begriffe im Dialekt mit zusätzlicher Umschrift und englischer Übersetzung enthalten. Außerdem wird die Aussprache aller Vokabeln als Audiodatei bereitgestellt. Jede Gruppe bein-

${ }^{181} \mathrm{Vgl}$. Council of Europe. 
haltet auch eine tägliche Frage, die zur Beantwortung in schriftlicher Form animieren soll. Zuletzt gibt es die Möglichkeit, Blog- und Tagebucheinträge zu lesen, welche Aspekte des alltäglichen Lebens in der arabischen Welt beschreiben und konkrete Tipps für Interaktionen in den arabischsprachigen Ländern geben.

Lingualism bietet viele Ressourcen für die selbstständige Erweiterung und Festigung der im Unterricht erworbenen sprachlichen Mittel und versucht darüber hinaus, kulturelle und gesellschaftliche Kenntnisse über die arabische Welt zu vermitteln. Zudem erlauben die Gruppen und Foren es Arabischlernenden, sich miteinander zu vernetzen und sich in den bereits erwähnten communities of practice, die für Lernende wichtige Vorteile bringen können, einzubringen. Hier wird auch mehr Interaktivität geschaffen, indem direkter Austausch über verschiedene Themen und linguistische Fragen stattfinden kann. Theoretisch bietet Lingualism durch die Kombination der Internetseite, des YouTube-Kanals und der Gruppen viele Möglichkeiten, das individuelle Sprachenlernen zu unterstützen. Jedoch muss hierbei bedacht werden, dass Schüler*innen über die entsprechende Motivation und Zeit verfügen müssen, um diese Möglichkeiten zu nutzen. Außerdem ist die Lingualism-Internetseite den sozialen Medien ähnlicher als traditionellen Lernplattformen, weshalb hier potenzielle Risiken auftreten können, beispielsweise die Vernetzung mit negativen Akteuren oder das unbedachte Teilen persönlicher Informationen. Dementsprechend sollte Lingualism unter Anleitung der Lehrkraft verwendet werden und potenzielle Risiken sowie angebrachtes Verhalten in Online-Foren sollten im Vorfeld besprochen werden. Letztlich ist zu bedenken, dass sich Lingualism an ein englischsprachiges Publikum richtet und Fragen vorrangig auf Englisch gestellt und beantwortet werden. Auch die Blogeinträge und Übersetzungen zu Musikvideos sind lediglich auf Englisch verfügbar. Um sich also aktiv in dieser Gemeinschaft zu beteiligen und von ihren Ressourcen zu profitieren, bedarf es guter bis sehr guter Englischkenntnisse. 


\subsection{Al Jazeeras Learning Arabic ${ }^{182}$}

Eine speziell für Lernende des Arabischen konzipierte Internetseite ist ta 'allum al'arabìya oder Learning Arabic von Al Jazeera ${ }^{183}$. Auf dieser Internetseite finden sich Übungen für Anfänger bis Fortgeschrittene, welche in die Lernbereiche General Language und Language of the Media unterteilt sind. Die verfügbaren Sprachniveaus sind genau abgesteckt und umfassen Beginner, Elementary, Intermediate, Upper Intermediate und Advanced, was die Suche nach passenden Übungen deutlich erleichtert. Hat man sich zwischen den Bereichen allgemeine Sprache und Sprache der Medien entschieden, werden verschiedene Aufgaben vorgestellt. Inhaltlich sind diese sehr abwechslungsreich und behandeln Themen wie aktuelle Nachrichten, historische Ereignisse, kulturelle Aspekte der arabischen Welt wie Kalligrafie, Literatur und Feiertage sowie Interviews mit arabischen Persönlichkeiten. Im Bereich Sprache der Medien finden sich hauptsächlich Zeitungsartikel oder Nachrichtenbeiträge von Al Jazeera. Auf dem Anfängerniveau gibt es Videos zu den arabischen Buchstaben, zur Aussprache grundlegender Wörter und kurze Dialoge, die teilweise mit arabischen Untertiteln und englischen und französischen Übersetzungen arbeiten. Ab der zweiten Stufe, Elementary, wird innerhalb der Videos nicht mehr mit Untertiteln oder Übersetzungen gearbeitet und der Schwierigkeitsgrad der Übungen liegt deutlich höher. Bis Niveaustufe Intermediate stellen Videos den Hauptteil der Aufgaben dar und der Fokus liegt auf Hörkompetenz. In den darauffolgenden Stufen Upper Intermediate und Advanced werden vorrangig Lesetexte bereitgestellt und die Aufgaben konzentrieren sich auf die Ausbildung von Lesekompetenz.

Die Internetseite Learning Arabic berücksichtigt einige konkrete Bedürfnisse Arabischlernender. Zum einen sind bis zur Stufe Intermediate alle Aufgabenstellungen und Überschriften voll vokalisiert, was beim Lesen und Verstehen der Angaben hilft. Die Vokalisierung kann aber auch ausgeschaltet werden, um das Lesen ohne Vokalzeichen zu unterstützen. Darüber hinaus beinhaltet jede Aufgabe eine vokalisierte Vokabelliste mit den wichtigsten Ausdrücken des zu bearbeitenden Videos oder Textes. Hier wird zwischen einzelnen Wörtern und Phrasen deutlich unterschieden und eine englische Übersetzung bereitgestellt. Jedes Video

\footnotetext{
${ }^{182}$ Learning Arabic ist verfügbar unter https://learning.aljazeera.net/en

${ }^{183}$ Vgl. Aljazeera Media Network 2020.
} 
wird auch mit dem jeweiligen, vollständigen Transkript auf Arabisch bereitgestellt, wobei das Transkript entweder vokalisiert oder unvokalisiert genutzt werden kann. Ein weiterer lobenswerter Aspekt der Al Jazeera Learning-Internetseite ist, dass jede Aufgabe, egal ob sie sich auf ein Video oder auf einen Lesetext bezieht, bis zu sechs verschiedene Übungen beinhaltet. Diese umfassen vielfältige Arbeitsaufträge wie das Verbinden von Satzteilen, die Beantwortung textbezogener Fragen, die Zuordnung bestimmter inhaltlicher Elemente $\mathrm{zu}$ passenden Überschriften, die Vervollständigung eines Lückentextes oder die Verwendung neuer Vokabeln in eigenen Sätzen. Bei Schreibaufgaben kann entweder mit der eigenen arabischen Tastatur geschrieben werden oder mit einer elektronisch bereitgestellten. Folglich bemüht sich die Internetseite auch, verschiedene Kompetenzbereiche, wie etwa Hören und Schreiben, gleichzeitig zu stärken und die Verwendung neuer Vokabeln in komplexeren Kontexten und Sätzen zu fördern.

Abgesehen von den einzelnen Hör- und Leseaufgaben bietet die Internetseite die Möglichkeit, das integrierte Wörterbuch zu verwenden, Fragen an eine Arabischlehrkraft zu stellen oder sich in der Community einzubringen. Diese bietet Arabischlernenden die Möglichkeit, Videos anzuschauen, die beispielsweise Tipps zum Lernen der arabischen Sprache geben, arabische Rezepte veranschaulichen oder die Erfahrungen anderer Arabischlernender auf der Welt vorstellen. Zuletzt gibt es auch die Möglichkeit, im Bereich Blogs Einträge zu Themen wie der Schreibung des Buchstaben hamza oder der Vorteile des Online-Sprachenlernens zu lesen.

Learning Arabic wird von Al Jazeera betrieben; somit ist grundsätzlich nicht von Neutralität auszugehen. Die angebotenen Videos und Texte werden von Al Jazeera produziert und fallen in die weitere Al Jazeera-Berichterstattung hinein. Auf der einen Seite muss hierbei bedacht werden, dass Al Jazeera als Nachrichtendienst oft in die öffentliche Kritik gerät und beispielsweise als Instrument katarischer Außenpolitik beschrieben wird. Dem Nachrichtendienst werden zudem die Finanzierung terroristischer Organisationen ${ }^{184}$ und eine einseitige Berichterstattung im Israel-Palästina-Konflikt vorgeworfen. ${ }^{185}$ Auf der anderen Seite ist nicht abzustreiten, dass vor allem die Vorwürfe bezüglich Subjektivität in der Bericht-

\footnotetext{
${ }^{184}$ Vgl. Ramadan 2017.

185 Vgl. Batrawy 2017.
} 
erstattung zu Israel-Palästina auf die meisten arabischen Nachrichtensender zutreffen würden. Zudem gehört Al Jazeera zu den größten Nachrichtendiensten der Welt und erreicht mit seiner Berichterstattung Millionen von Menschen, vor allem in den arabischen Ländern. ${ }^{186}$ Darüber hinaus ist zu bedenken, dass jedes Nachrichtenportal, das im Unterricht Verwendung findet, kritisch reflektiert werden sollte und dass auch andere Anbieter von Sprachlernmaterialien und programmen nicht als wertfrei gelten können. Das Goethe-Institut beispielsweise agiert aufgrund seiner Beziehung zum Auswärtigen Amt nicht nur als Institution für Deutschunterricht, sondern auch als Mittlerorganisation deutscher Kulturpolitik. ${ }^{187}$ Es wäre der kritischen Medienbildung im Unterricht nicht zuträglich, $A l$ Jazeeras Inhalte einfach zu ignorieren. Vielmehr könnte die Benutzung von Learning Arabic eine Möglichkeit sein, die Berichterstattung in der arabischen Welt näher zu besprechen und mit den Schüler*innen potenzielle Kritikpunkte zu diskutieren. Man könnte die Videos und Artikel auf Learning Arabic also nicht nur nutzen, um sprachliche Mittel zu festigen, sondern auch, um mediale Inhalte kritisch $\mathrm{zu}$ beleuchten und Voreingenommenheit $\mathrm{zu}$ erkennen und $\mathrm{zu}$ thematisieren.

Für die Übungen auf den niedrigen Sprachniveaus sind Englischkenntnisse teilweise notwendig. Wenn aber bereits ausreichende Arabischkenntnisse vorhanden sind, dann sollten auch deutsche Schüler*innen die Internetseite problemlos nutzen können. Da die Aufgabenstellungen innerhalb der Übungen oft gleich formuliert sind, sollte eine kurze Anleitung genügen, damit Schüler*innen Learning Arabic auch selbstständig verwenden können. Die angebotenen Videos und Lesetexte zeichnen sich vor allem durch abwechslungsreiche Inhalte und viele tagesaktuelle Themen aus. Durch den gut strukturierten Aufbau ist es nicht schwierig, Übungen nach Sprachniveau oder Themenbereich auszuwählen, weshalb Schüler*innen hier je nach Bedürfnissen und Interessen Prioritäten setzen können. Auch für die Lehrkraft erleichtert die übersichtliche Navigation der Internetseite die Auswahl von Materialien und Übungen für den Unterricht.

Ein Vorteil von Learning Arabic ist, dass alle Videos mit Transkripten zur Verfügung gestellt werden, was zusätzliche Unterstützung bei Hörverständnis-

\footnotetext{
${ }^{186} \mathrm{Vgl}$. Kühn et al. 2013.

187 Vgl. Sommer 2020.
} 
aufgaben bietet. Zudem fördern die angebotenen Übungen nicht nur die Kompetenzen Lesen, Hören und Schreiben, sondern beinhalten oft auch wichtige und gut aufbereitete kulturelle und historische Informationen. Außerdem zeichnen sich die Themenbereiche auf den höheren Sprachniveaus durch die Verwendung qualitativ hochwertigen, authentischen Materials aus. Die Möglichkeit, sich in der Community einzubringen oder Fragen an eine Arabischlehrkraft zu stellen, trägt zu einem gewissen Grad an Interaktivität bei. Darüber hinaus sind die verfügbaren Übungen leicht und auf mehreren Wegen teilbar. Ein wichtiger Aspekt von Learning Arabic für Lehrkräfte ist, dass im Vergleich zu anderen Portalen, auf denen einzelne Lehrer*innen Materialien ohne nochmalige Prüfung hochladen können, die Inhalte und Übungen auf Learning Arabic professionell erstellt und regelmäßig aktualisiert werden. So kann grundsätzlich von sprachlicher Korrektheit ausgegangen werden. Ein potenzieller Nachteil der Seite Learning Arabic ist, dass keine Inhalte in den arabischen Dialekten angeboten werden und die Materialien ausschließlich zur Festigung des Hocharabischen beitragen können.

\subsection{Playaling ${ }^{188}$}

Das Konzept von Playaling ist simpel und dennoch erstaunlich effektiv: Die Internetseite bietet eine Vielzahl arabischsprachiger Videos mit arabischen Transkripten und englischen Untertiteln. Playalings Ziel ist es, authentische und anwendungsorientierte Inhalte verfügbar zu machen, die einen didaktischen Mehrwert für Lernende des Arabischen darstellen können. Der didaktische Ansatz liegt darin, dass Lernende sich an der Sprechweise von Muttersprachler*innen orientieren sollen, um selbst funktionale kommunikative Fähigkeiten $\mathrm{zu}$ erwerben. Laut Gründer der Internetseite Playaling geht es hier vor allem darum, konkrete Beispiele für das sogenannte Educated Spoken Arabic (ESA) bereitzustellen ${ }^{189}$, welches von gebildeten Arabischmuttersprachler*innen gesprochen wird und sich durch die parallele Verwendung hocharabischer und dialektaler Elemente auszeichnet. Folglich können gebildete Muttersprachler*innen ihre Sprechweise je nach sozialem, kulturellem und politischem Kontext anpassen und verschiedene

\footnotetext{
188 Playaling ist verfügbar unter https://playaling.com/

${ }^{189}$ Vgl. Playaling (b).
} 
Sprachregister ausdrücken. ${ }^{190}$ Laut Playalings Internetseite ist die Orientierung an ESA eine Möglichkeit, dem ausschließlichen Unterrichten von Hocharabisch entgegenzuwirken und Schüler*innen auf echte, alltägliche kommunikative Situationen vorzubereiten. Da ESA ein weitgefasster Begriff ist und je nach Land, Region, Situation, Gesprächspartner und eigenen Sprachkenntnissen anders aussehen kann, versucht Playaling grundsätzlich, so viele verschiedene sprachliche Beispiele wie möglich verfügbar zu machen. Dies soll Lernende auch dazu befähigen, bedürfnisbezogen zu wählen. ${ }^{191}$

Die Videos auf Playaling können nach Sprachniveau, Inhalt und sprachlicher Varietät - Hocharabisch, einige der arabischen Dialekte oder ESA - gefiltert werden. Alle Videos sind mit interaktiven Untertiteln ausgestattet, welche das Gesprochene genauestens transkribieren. Ist der Inhalt des Videos im Dialekt gesprochen, so sind auch die dazugehörigen Untertitel im Dialekt. Diese sind jedoch optional und können jederzeit ein- oder ausgeschaltet werden. Manche Videos beinhalten zudem die Funktion, dialektale Inhalte in hocharabische Untertitel umzuwandeln. Darüber hinaus gibt es zu jedem Video Untertitel auf englischer Sprache und eine kurze Einführung in den Inhalt des Videos. Die verfügbaren Niveaustufen sind Novice, Beginner, Intermediate und Advanced und es kann unter den Dialekten aus Ägyptisch, Levantinisch und Nordafrikanisch gewählt werden. Nicht alle Videos auf der Internetseite sind als ESA kategorisiert, sondern lediglich solche, bei denen tatsächlich eine konsequente Mischung von Hocharabisch und Dialekt stattfindet. In diesen Fällen ist vorher angegeben, um welchen Dialekt es sich handelt. Trotzdem gibt es beispielsweise Videos im levantinischen Dialekt, welche auch Elemente des Hocharabischen enthalten und Videos auf Hocharabisch, in denen dialektale Ausdrücke verwendet werden. Die angebotenen Themenbereiche sind vielfältig und reichen unter anderem von Geschichte, Alltag, Religion und Kultur bis zu „ausgefallen und kontrovers“ und „zum Wohlfühlen und inspirierend“. Die Inhalte sind verschiedenen Quellen entnommen und umfassen Nachrichtenbeiträge, Musikvideos, Ausschnitte aus Interviews, Talkshows und Fernsehserien und animierte Videos für Kinder.

\footnotetext{
${ }^{190}$ Vgl. Ryding 2015, S. 74.

${ }^{191}$ Vgl. Playaling.
} 
Das Alleinstellungsmerkmal von Playaling ist, dass die arabischen Untertitel nicht einfach nur den gesprochenen Inhalt transkribieren, was an sich schon eine wichtige Errungenschaft ist, da genaue arabische Untertitel auch auf anderen Internetseiten schwer zu finden sein können, sondern, dass jedes Wort markiert werden kann und folglich die Radikale des Wortes bzw. die Wortwurzel angezeigt wird. Außerdem wird die Übersetzung des Wortes auf Englisch vorgegeben und es werden etwaige Wortverwandtschaften eines dialektalen Ausdrucks zum Hocharabischen erklärt. Zudem werden wichtige grammatische Aspekte des Wortes, wie beispielsweise die Verwendung der Nunation, kurz erläutert. Für Substantive wird oft der Plural mit angegeben, Eigennamen werden als solche hervorgehoben und zusammenhängende Wortgruppen werden auch zusammenhängend übersetzt und erklärt. Auch Genitivverbindungen werden teils zusammen übersetzt, was dabei hilft, die einzelnen Satzteile und ihre entsprechenden Funktionen zu erkennen.

Bezüglich der Kriterien für sinnstiftende Bildungsmedien des vzbv tut sich Playaling vor allem im Bereich „Methodik und Didaktik“ hervor, da die verfügbaren Inhalte zeitgemäß sind und sich an den Interessen junger Lernender orientieren. Zudem behandeln viele der Videos auf Playaling kontroverse Inhalte, wie beispielsweise das mehrteilige Interview mit der libanesischen Band Mashrou Leila ${ }^{192}$, welches Themen wie Homosexualität, Kritik an politischen Systemen und Jugendarbeitslosigkeit in der arabischen Welt anspricht. Außerdem hilft der kurze Einleitungstext zum Thema des Videos dabei, Schüler*innen mental und sprachlich vorzubereiten und bereits vor Anschauen des Videos Assoziationen zu finden und themenrelevantes Vokabular zu reaktivieren. Obwohl das Hauptziel von Playaling ist, die Hörkompetenz der Lernenden zu verbessern, eignen sich die Videos und ihre Transkriptionen auch als Ausgangspunkt für weitere Arbeitsaufträge: Die Erklärung der Wortwurzeln kann beispielsweise zu weiterführender Wörterbucharbeit animieren, es kann innerhalb der Transkripte nach Wortverwandtschaften gesucht werden, die Transkripte, welche unvokalisiert sind, können zur Einübung der Kasusendungen und Vokalzeichen dienen und die vielfältigen Inhalte der Videos können zu Ausgangspunkten für Diskussionen oder Aufsätze werden. Playaling hat den Vorteil, dass es authentische Inhalte durch genaue Transkription und zusätzliche linguistische Anmerkungen didaktisch aufbereitet

${ }^{192}$ Der erste Teil des Interviews ist verfügbar unter https://playaling.com/preview/159 
und so auch für Schüler*innen auf dem Anfängerniveau nützlich ist. Die Internetseite selbst enthält keine Werbung oder tendenziösen Darstellungen. Die Inhalte sind unverändert aus den ursprünglichen Quellen übernommen und sind insofern sachrichtig und unparteiisch, als dass sie in ihrer Originalform verfügbar gemacht werden.

Playaling eignet sich als Quelle für Audiomaterial auf Hocharabisch und in den Dialekten. Hinzu kommt die Tatsache, dass besonderer Wert auf authentische Texte gelegt wird, welche die Kombination mehrerer Varietäten zeigen und somit als Ressource für die Ausbildung größerer Sprachbewusstheit dienen können. Schüler*innen erhalten durch die Videos Einblicke in verschiedene soziokulturelle Kontexte und Medienformate und können analysieren, weshalb bestimmte Varietäten zu bestimmten Zeiten und an bestimmten Orten verwendet werden. Die Seite ist sehr einfach aufgebaut und sollte sowohl für die Lehrkraft als auch für die Schüler*innen leicht zu navigieren sein. Playaling eignet sich gut für individuelles Lernen außerhalb des Unterrichts, da viele relevante Informationen bereits durch die Internetseite bereitgestellt werden und Lernende die verwendeten Wörter und Ausdrücke selbstständig näher analysieren können. Da die Untertitel und Anmerkungen nur auf Englisch vorhanden sind, benötigen die Schüler*innen jedoch ein adäquates Englischniveau, um vor allem die linguistischen Hinweise zu verstehen. Andererseits könnten deutschsprachige Lernende auch von vornherein die englischen Untertitel ignorieren und lediglich mit den arabischen Transkripten sinnvoll arbeiten.

\subsection{Khallina ${ }^{193}$}

Khallina beschreibt sich selbst als ein Open-Source-Tool, mit dem Arabischlehrer*innen und Lernende die arabische Kultur entdecken können. ${ }^{194}$ Die Internetseite bietet fünfzehn Module auf drei verschiedenen Sprachniveaus an - Novice, Intermediate und Advanced. Jedes Modul widmet sich einem bestimmten kulturellen Thema. Die Inhalte sind abwechslungsreich und umfassen beispielsweise die Bedeutung von Brot in der arabischen Welt, Comics und grafische Romane, die

\footnotetext{
${ }^{193}$ Khallina ist verfügbar unter https://khallina.org/

${ }^{194}$ Vgl. Khallina 2020a.
} 
Teilhabe der Frau in den arabischen Ländern, marokkanisches Kunsthandwerk und alternative Musik. Die Module setzen sich aus verschiedenen Schritten zusammen, wobei jeder Schritt einen anderen Arbeitsauftrag enthält und durch die Verwendung von Videos, Zeitungsartikeln, grafischen Darstellungen und grammatischen Erklärungen multimediale, abwechslungsreiche Module geschaffen werden. Jedes Modul verweist abschließend auch auf weitere Ressourcen für die Vertiefung der Inhalte und das eigenständige Lernen.

Khallinas Themenspektrum richtet sich deutlich an ein jüngeres Publikum und ist inhaltlich für den schulischen Kontext relevant. In den Modulen auf dem Anfängerniveau wird noch viel Englisch verwendet, während die Inhalte auf den folgenden Niveaus fast ausschließlich auf Arabisch sind. Der Aufbau eines typischen Moduls innerhalb Khallinas kann am Beispiel von Women Empowerment auf dem Sprachniveau Intermediate ${ }^{195}$ verdeutlicht werden:

Das Modul beginnt mit einer kurzen Einführung in das Thema auf Englisch und einer Liste von angestrebten Lernzielen. In diesem Falle gehört dazu beispielsweise das Kennenlernen einflussreicher arabischer Frauen und Frauenbewegungen in den arabischen Ländern. Danach steigen die Lernenden in das Modul ein, welches sich aus zehn Schritten zusammensetzt. Der erste Schritt beginnt oft mit einem Lesetext, in diesem Falle werden die Kurzbiografien vierzehn bekannter Frauen aus der arabischen Welt vorgestellt und sollen den entsprechenden Bildern zugeordnet werden. Im zweiten Schritt geht es weiter mit einem Video, aus welchem wichtige neue Vokabeln extrahiert werden sollen, um sie im Folgenden in einem eigenen Text zu verwenden. Im dritten Schritt finden die Lernenden die integrierte Karteikartenfunktion und können so einige neue Vokabeln lernen und festigen. Außerdem werden hier ein neues Video und eine Frage präsentiert, die zu weiteren Diskussionen anregen sollen. In diesem Modul beispielsweise sollen die Schüler*innen diskutieren, wie sie über typisch männliche und typisch weibliche Berufe denken. Im vierten Schritt folgt ein Video von Al Jazeera, welches die erste Lokführerin in den Emiraten vorstellt. Das Video ist hauptsächlich auf Hocharabisch und beinhaltet ein paar dialektale Elemente. Dieser sprachliche Aspekt unterscheidet sich von Modul zu Modul, wobei manche Module vorrangig Inhalte

${ }^{195}$ Vgl. Khallina 2020b. 
auf Hocharabisch vorstellen, während andere Inhalte in der Umgangssprache priorisieren. Die Lernenden sollen hier das Video mehrmals anschauen und zwei Lückentexte vervollständigen. Im fünften Schritt werden den Lernenden mehrere Annoncen für freie Stellen in der arabischen Welt präsentiert und es soll analysiert werden, ob speziell nach Frauen gesucht wird und inwiefern diese Stellenanzeigen versuchen, Frauen professionell zu fördern. Der sechste Schritt heißt 'āmmīya'196 und bezieht sich auf die dialektalen Aspekte, die im vorigen Video zu erkennen waren. Hier wird erklärt, was sogenannte loan verbs sind, also Verben, die aus dem Englischen in das umgangssprachliche Arabisch übernommen werden. Da die Erklärungen auf Englisch sind, müsste die Lehrkraft im deutschen Kontext entsprechend übersetzen und möglicherweise eine eigene Erklärung auf Deutsch erstellen. Im siebten Schritt folgen Erläuterungen zur Grammatik des Imperativ. Auch hier ist der Großteil der Erklärung auf Englisch und müsste für deutschsprachige Schüler*innen eventuell angepasst werden. Im achten Schritt folgt eine Wiederholung der wichtigsten Vokabeln. Der neunte Schritt namens dawrak („Du bist dran!“) beinhaltet normalerweise eine konkrete Aufgabenstellung, welche die Schüler*innen dazu auffordert, ihr erworbenes Wissen kreativ anzuwenden. In diesem Modul lautet der Auftrag beispielsweise, eine Kampagne zu erstellen, die durch Slogans, Bilder und andere grafische Darstellungen auf die Diskriminierung von Frauen aufmerksam macht. Der zehnte und letzte Schritt beinhaltet weitere Ressourcen zum Thema, in diesem Falle unter anderem den Trailer zum saudiarabischen Film Wajda, das Musikvideo zu Bashar Murads Lied Ana Zalameh (,Ich bin ein Mann“) und Interviews mit verschiedenen Frauen aus der arabischen Welt, die sich zum Thema äußern.

Khallina sticht unter den frei verfügbaren Ressourcen für das Erlernen bzw. die Vermittlung der arabischen Sprache aus mehreren Gründen hervor. Zum einen wird deutlich, dass Khallina den bereits in Kapitel 4 erwähnten Ansatz, dass kulturelles und soziokulturelles Wissen im Arabischunterricht einen festen Platz einnehmen sollte, umsetzt und anstrebt, die arabischsprachige Welt aus verschiedenen Perspektiven zu präsentieren. Die Lesetexte und Videos versuchen, Schüler*innen den gesellschaftlichen Kontext näherzubringen und einseitigen Darstellungen der arabischen Gesellschaften entgegenzuwirken. Inhalte werden auf moderne und

\footnotetext{
${ }^{196}$ Das Wort 'āmmīya beschreibt auf Arabisch die Umgangssprache bzw. die arabischen Dialekte.
} 
zeitgemäße Art vermittelt und richten sich vorrangig an die Interessen und Bedürfnisse von Jugendlichen und jungen Erwachsenen. Die grafische Gestaltung auf der Internetseite Khallina ist deutlich hochwertiger als auf anderen Lernplattformen und zeichnet sich durch eine Mischung aus Diagrammen, Bildern und Videos sowie einem leicht verständlichen und intuitiven Navigationsmenü aus. Die Gliederung in drei Sprachniveaus erleichtert die Suche nach passenden Modulen. Der Einführungstext eines jeden Moduls hilft dabei, Schüler*innen die konkreten Lernziele $\mathrm{zu}$ verdeutlichen und sie auf die folgenden Inhalte des Moduls vorzubereiten. Die Übungen innerhalb der Module sind methodisch abwechslungsreich und zielen auf die Ausbildung verschiedener Kompetenzen ab, wobei vor allem Hör- und Leseverstehen durch die vielfältigen Videos und Lesetexte unterstützt werden. Gleichwohl wird auch versucht, Schreib- und Sprechkompetenz zu fördern, beispielsweise durch kontroverse Thesen oder Fragen, die in mündlichen Diskussionen oder schriftlichen Aufsätzen behandelt werden sollen. Alle Aufgaben auf Khallina können auch als Arbeitsblätter heruntergeladen und ausgedruckt werden und beinhalten sowohl individuelle Arbeitsaufträge als auch Übungen, die in Gruppen bearbeitet werden sollen. Die Internetseite präsentiert keine Werbung oder Links zu kommerziellen Anbietern.

Der Aufbau von Khallina orientiert sich an den verschiedenen Phasen, die auch eine normale Unterrichtsstunde durchlaufen würde. Innerhalb der Module von Khallina ist eine deutliche Progression von der Präsentation neuer Inhalte zur Übung dieser Inhalte bis hin zur Anwendung ${ }^{197}$, vor allem im Schritt dawrak, zu erkennen. Es kann argumentiert werden, dass die Phasen Einübung und Transfer, welche vor der eigenständigen, kreativen Anwendung neuer Inhalte stehen ${ }^{198}$, auf Khallina zu kurz kommen, da der Präsentation neuer Vokabeln und grammatischer Inhalte kaum Möglichkeiten folgen, diese auch zu üben. Hier zeigt sich ein wichtiger Aspekt der Verwendung neuer Medien im Unterricht, nämlich dass das Medium selbst, sei es noch so professionell gestaltet und pädagogisch durchdacht, die Lehrkraft nicht ersetzen kann oder sollte, sondern lediglich als zusätzliches Werkzeug und nützliche Ressource dient. ${ }^{199}$ Es fällt der Lehrkraft immer noch zu, einen sinnstiftenden, abwechslungsreichen und kompetenzorientierten Unterricht

\footnotetext{
${ }^{197}$ Vgl. Nieweler 2017, S. 195.

${ }^{198}$ Vgl. ebd.

${ }^{199}$ Vgl. Madhany 2006, S. 301.
} 
zu gestalten und die Defizite, die sich unweigerlich in jeder Form von Bildungsmedium finden werden, wettzumachen. Es empfiehlt sich beispielsweise, Übungen und Arbeitsblätter zu gestalten, die Schüler*innen verstärkt zur Benutzung der neuen Inhalte von Khallina anhalten.

Ein Alleinstellungsmerkmal von Khallina ist in jedem Falle die Behandlung vielfältiger, teilweise kontroverser Themen auf multiperspektivische Art. Unter den fünfzehn Modulen findet man beispielsweise auf dem Sprachniveau Novice das Modul Comix and Graphic Novels, in welchem unter anderem behandelt wird, inwiefern Comics als Werkzeug für Systemkritik dienen und wie grafische Romane gesellschaftliche Probleme wie die Belästigung von Frauen ansprechen können. Das Modul Bread lässt vom Titel her auf wenig kontroversen Inhalt schließen, befasst sich aber unter anderem damit, welche Rolle das Wort und das Konzept Brot in der ägyptischen Revolution gespielt haben. Im Modul Across Borders geht es um das Thema Migration und Flucht und im Modul Civil Society erfahren Lernende mehr über zivilgesellschaftliche Initiativen in der arabischen Welt, die Problemfelder wie Frauen- und Arbeiterrechte thematisieren.

\subsection{Ergebnisse des Vergleichs}

Wie sich deutlich zeigt, verfügt jede der analysierten Internetseiten und Plattformen über besondere Vor- und Nachteile und kann bestimmte sprachliche Kompetenzen und Lernziele sinnvoll unterstützen. Keines der verglichenen Medien kann in gleichem Maße alle Bereiche des Arabischunterrichts unterstützen und es sollte zielgerichtet und bedürfnisgebunden gewählt werden. Ein Problemfeld in der Vermittlung der arabischen Sprache, welches beispielsweise durch InterLingo, Lingualism, Learning Arabic und Playaling vereinfacht werden kann, ist das Fehlen von Transkripten für arabisches Audiomaterial. Laut van Mol liegt nämlich eine besondere Schwierigkeit der arabischen Sprache für europäische Lernende darin, dass sie generell viele Synonyme beinhaltet und dass es wenige Lehnwörter aus dem Englischen oder anderen lateinischen Sprachen gibt, die man in gesprochenen oder geschriebenen Texten schnell identifizieren und verstehen kann. Diese Gegebenheiten führen dazu, dass Arabischlernende vor allem bei 
Höraufgaben schnell überfordert sein können. ${ }^{200}$ Hinzu kommt eine generelle Schnelligkeit in authentischen Texten wie Nachrichtenbeiträgen oder Talkshows, welche jedoch keinesfalls exklusiv auf Arabisch zutrifft. Dementsprechend müssen authentische Hörtexte wie Radio- und Fernsehprogramme mit Transkripten bereitgestellt werden, um den Lernenden Anhaltspunkte zu bieten und das Hörverstehen unter Zuhilfenahme von verschriftetem Material zu schulen. ${ }^{201}$ Während Learning Arabic und InterLingo vorrangig Hörtexte und Transkripte auf Hocharabisch anbieten, stellen Lingualism und Playaling auch Transkripte für Videos und Hörtexte in den Dialekten bereit.

Lingualism, Playaling, Khallina und die Ressourcen des DLIFLC sind zudem für die Überwindung einer zweiten Hürde im Bereich AraF empfehlenswert, nämlich dem Fehlen von Lehrmaterialien für die Dialekte. ${ }^{202}$ Hinzu kommt, dass beispielsweise Playaling und Khallina, welche Videos in verschiedensten Sprachregistern anbieten und viele Inhalte verfügbar machen, in denen Hocharabisch und Dialekte authentisch gemischt werden, auf deutsche Schüler*innen besonders motivierend wirken $^{203}$ und zur Ausbildung der essenziellen Kompetenz Sprachbewusstheit beitragen könnten. ${ }^{204}$

Wie in Kapitel 4 beschrieben, plädiert unter anderem Turner für die verstärkte Vermittlung kulturellen Wissens. ${ }^{205}$ Auch Behzadi et al. merken an, „dass ein Arabischunterricht, der nicht auch kulturellen, landeskundlichen und aufklärerischreligiösen Inhalten einen angemessenen Raum einräumt, (Bildungs-)Potenziale auf Seiten der Schülerinnen und Schüler verschenkt. “" ${ }^{206}$ Um diesen Ansatz zu verwirklichen, bietet sich vor allem die Verwendung der Internetseite Khallina an, welche innerhalb der Module verschiedene gesellschaftliche, politische, kulturelle und landeskundliche Themen aufgreift und kontroversen Diskussionen nicht aus dem Weg geht, sondern sie gezielt anregt. Auch Learning Arabic und Lingualism stechen durch ihre Themenvielfalt und die vielfältigen Informationen über die ara-

\footnotetext{
${ }^{200}$ Vgl. Van Mol 2006, S. 306.

201 Vgl. ebd., S. 308.

202 Vgl. Snowden und Soliman, Rasha, Towler, Melissa 2016, S. 14.

${ }^{203}$ Vgl. Behzadi et al. 2019, S. 414.

${ }^{204}$ Vgl. Ständige Konferenz der Kultusminister der Länder in der Bundesrepublik Deutschland

2011, S. 4

${ }^{205}$ Vgl. Turner 2018, S. 149f.

${ }^{206}$ Vgl. Behzadi et al. 2019, S. 421.
} 
bischen Länder, vorrangig von Muttersprachler*innen in authentischen Videos und Hörtexten präsentiert, hervor. Die Verwendung dieser Internetseiten kann einerseits zur geforderten Vermittlung wichtiger kultureller Aspekte im Arabischunterricht beitragen und andererseits Schüler*innen dazu anregen, sich mit ihrer eigenen Kulturgebundenheit auseinanderzusetzen und mit gesellschaftlicher Komplexität umzugehen. ${ }^{207}$

Innerhalb des Vergleichs wird deutlich, dass die Lehrkraft nicht nur in der Auswahl der verfügbaren Medien eine wichtige Rolle spielt, sondern auch in der Anleitung der Schüler*innen. Einige der vorgestellten Medien könnten ohne Einführung und zusätzliche Erklärungen der Lehrkraft nicht sinnvoll verwendet werden. Zudem zeigt sich, dass viele digitale Medien und deren Inhalte nicht direkt in den Arabischunterricht übernommen werden können, sondern oftmals einer Anpassung, Umarbeitung oder Übersetzung seitens der Lehrkraft bedürfen.

${ }^{207}$ Vgl. Behzadi et al. 2019, S. 407f. 


\section{Theoretische Unterrichtseinheit}

Die wichtigste Erkenntnis, die sich innerhalb der Recherche zum Thema Medienbildung im Fremdsprachenunterricht und durch die Analyse der Arabischlehrpläne gezeigt hat, ist, dass dem Lernen mit Medien viel größere Bedeutung zukommt als dem Lernen über Medien. Die kritische Auswahl verfügbarer Medien, die Reflexion ihrer Vor- und Nachteile und die kompetente Anleitung von Schüler*innen in der bedürfnisbezogenen und altersgerechten Auswahl neuer Medien kommt in allen Lehrplänen, mit Ausnahme des Lehrplans aus New South Wales, deutlich zu kurz oder wird gar nicht erwähnt. Auch in den vorgestellten Anwendungsbeispielen und -vorschlägen werden digitale Medien fast ausschließlich als Hilfsmittel für bestimmte Aufgabenstellungen verwendet, während ihre kritische Bewertung und Betrachtung kaum angestrebt werden. Zudem wird in den meisten Lehrplänen und Anwendungsbeispielen kein pädagogisches Konzept für die Verwendung neuer Medien erläutert. Wie die KMK jedoch betont, muss der Einsatz digitaler Medien im Unterricht dem Primat des Pädagogischen folgen. Dementsprechend soll im letzten Teil dieser Forschungsarbeit ein theoretisches Konzept für eine Unterrichtseinheit vorgelegt werden, welche diese Probleme aufgreift und potenzielle Lösungsansätze präsentiert. Um sicherzustellen, dass die vorgeschlagenen Inhalte und Aktivitäten klare pädagogische Ziele verfolgen, wurde die Unterrichtseinheit unter Zuhilfenahme der Prinzipien der kritisch-konstruktiven Didaktik nach Klafki ${ }^{208}$ und des Lehrphasenmodells von Zimmermann ${ }^{209}$ entworfen. Der präsentierte Vorschlag ist ausschließlich theoretischer Natur und wurde noch nicht in der Praxis umgesetzt oder empirisch geprüft.

\subsection{Zielgruppe}

$\mathrm{Zu}$ Beginn soll beschrieben werden, welches konkrete Szenario bei der Konzipierung der Unterrichtseinheit bedacht wurde. Die Unterrichtseinheit richtet sich an Lernende des Arabischen als Fremdsprache an einer Sekundarschule, welche sich hinsichtlich ihrer Kenntnisse des Hocharabischen auf Niveau B1 laut Global-

\footnotetext{
208 Vgl. Klafki 2007, S. 272.

${ }^{209}$ Vgl. Zimmermann 1988, zitiert nach Nieweler 2017, S. 194-196.
} 
skala des Europäischen Referenzrahmens ${ }^{210}$ befinden. Sie beschäftigen sich bereits seit mehreren Jahren mit der arabischen Sprache und der Arabischunterricht findet öfter als einmal pro Woche statt. Die Schüler*innen sind Jugendliche über 14 Jahre. In Bezug auf die Klassengröße sollte die Unterrichtseinheit flexibel eingesetzt werden können und es kann durchaus in Betracht gezogen werden, die beschriebene Progression über einen längeren Zeitraum hinweg zu durchlaufen, beispielsweise innerhalb von ein oder zwei Wochen.

\subsection{Thema}

Der Ansatz der kritisch-konstruktiven Didaktik gliedert sich in fünf Grundfragen, wobei die ersten drei Grundfragen vor allem der Legitimation des im Unterricht behandelten Themas dienen. In der ersten Grundfrage geht es um die Gegenwartsbedeutung des ausgewählten Themas für die Schüler*innen. ${ }^{211}$ Für diese Unterrichtseinheit wurde das Thema soziale Medien gewählt, mit der Begründung, dass soziale Medien eine wichtige Rolle im Alltag der jugendlichen Schüler*innen spielen und ihnen das Thema so bereits aus dem außerschulischen Kontext bekannt ist. Zudem könnte man sich vorstellen, dass das Thema der sozialen Medien möglicherweise selbst von den Schüler*innen im Unterricht aufgeworfen wurde und dass beispielsweise gefragt wurde, ob und wie Menschen in der arabischsprachigen Welt soziale Medien verwenden. In diesem Falle wäre dieses Thema sowohl eine Möglichkeit, Schüler*innen an einem Punkt in ihrer Lebenswirklichkeit abzuholen, der ihnen bereits vertraut ist, und zudem auf ihre artikulierten Interessen einzugehen. Darüber hinaus ist das Thema pädagogisch wertvoll, da der Umgang mit sozialen Medien Chancen und Gefahren birgt, welche innerhalb des Unterrichts angesprochen werden sollen. Folglich erhalten die Schüler*innen ein besseres Verständnis über die Rolle und den Effekt sozialer Medien in unserer Gesellschaft und werden zugleich zur Selbstreflexion in Bezug auf ihre eigene alltägliche Verwendung sozialer Medien angehalten.

${ }^{210}$ Vgl. Council of Europe.
${ }^{211}$ Vgl. Klafki 2007, S. 273. 
Die zweite Grundfrage bezieht sich auf die Zukunftsbedeutung des Themas ${ }^{212}$ und inwieweit es Kulturtechniken aufzeigen kann, die für zukünftige private und berufliche Zwecke wichtig sind. ${ }^{213}$ Wie bereits in der Einleitung erwähnt, ist die Verwendung digitaler Medien in unserer Gesellschaft zu einer wichtigen Kulturtechnik geworden, welche Rechnen und Schreiben gleichwertig ist. Die Implikation ist, dass junge Menschen Medien kompetent und verantwortlich verwenden können müssen, um sich erfolgreich in unserer Gesellschaft einzubringen. Zudem steigen die Bedeutung des Internets und der sozialen Medien stetig an und es ist davon auszugehen, dass in Zukunft noch mehr soziale Medien verfügbar sein werden, welche durch die Weiterentwicklung mobiler Endgeräte zunehmend jeden Aspekt des alltäglichen Lebens beeinflussen werden. Soziale Medien werden zudem sowohl für private als auch für berufliche Zwecke eingesetzt, weshalb ihre verantwortungsvolle Verwendung auf beiden Ebenen von Bedeutung ist.

Die dritte didaktische Grundfrage bezieht sich auf die exemplarische Bedeutung des Themas. Konkret wird hier gefragt, auf welchen allgemeinen Sachverhalt das Thema hinweist und welches übergreifende Problem dadurch behandelt wird. Es sollen sich unter anderem Zusammenhänge, Gesetzmäßigkeiten, Strukturen und Widersprüche erarbeiten lassen. ${ }^{214}$ Das Thema der sozialen Medien ist hier didaktisch gerechtfertigt, weil es die größere Frage der Meinungsfreiheit und Teilhabe am politischen und gesellschaftlichen Diskurs behandelt und beispielsweise Widersprüche wie freie Meinungsäußerung im digitalen Raum und Zensur sowie die Zusammenhänge zwischen digitaler Kommunikation und dem Verlust zwischenmenschlicher Kontakte darstellt.

Im Folgenden war zu überlegen, wie die entworfene Unterrichtseinheit aufgebaut sein sollte und welche Struktur eine nachvollziehbare und sinnvolle Progression von der Präsentation des neuen Themas über das Aufnehmen neuer themenbezogener und sprachlicher Informationen bis hin zur Einübung und eigenständigen Anwendung des erworbenen Wissens bietet. Es wurde entschieden, die Unterrichtseinheit entsprechend der von Zimmermann beschriebenen fünf Phasen des fremdsprachlichen Grammatikunterrichts ${ }^{215}$ aufzubauen. Obwohl diese ursprüng-

\footnotetext{
${ }^{212}$ Vgl. Klafki 2007, S. 273-275.

${ }^{213}$ Vgl. Vormbaum.

${ }^{214}$ Vgl. Klafki 2007, S. 275.

${ }^{215}$ Vgl. Zimmermann 1988, zitiert nach Nieweler 2017, S. 194-196.
} 
lich für die Vermittlung grammatischer Kenntnisse entworfen wurden, werden sie mittlerweile auch für weitreichendere Unterrichtszwecke verwendet.

Die fünf Phasen setzen sich wie folgt zusammen: Präsentation, Einübung, Transfer, Anwendung und Kognitivierung. In der Präsentationsphase geht es darum, die neuen, bisher unbekannten Strukturen in kommunikativen Situationen oder zumindest in situativen Zusammenhängen zu präsentieren. Dies beinhaltet beispielsweise auch die Präsentation neuer Vokabeln und deren Nachsprechen. ${ }^{216}$ Die Einübung dient der aktiven Anwendung der neuen Inhalte. Es geht vorrangig um das „Geläufigmachen“ der neuen Konzepte und Ausdrücke ${ }^{217}$ sowie die Vermittlung der wichtigen Merkmale neuer grammatischer Strukturen. ${ }^{218}$ Auf die Einübung folgt der Transfer, in welchem die Anwendbarkeit der neuen Strukturen in noch ungeübten Minimalsituationen erzielt werden soll. ${ }^{219}$ Diese Phase soll ein produktives, kreatives Verhalten fördern und kognitive Entscheidungen erforderlich machen. ${ }^{220}$ Zuletzt folgt die Anwendungsphase, in welcher die neuen Strukturen komplett eigenständig verwendet werden sollen. ${ }^{221}$ Hier wird mehr Fokus auf den Inhalt sprachlicher Äußerungen als auf die neue Struktur selbst gelegt. ${ }^{222}$ Die Anwendung findet häufig nicht im Unterricht, sondern als Teil der Hausaufgabe statt.

Die Kognitivierungsphase ist als einzige flexibel anzuordnen und beinhaltet konkrete Erklärungen zu neuen grammatischen Strukturen, entweder durch die Lehrkraft, ein Lehrwerk oder durch Erarbeitung der Schüler*innen unter Anleitung der Lehrkraft. Diese Phase passt jedoch am sinnvollsten in die Präsentation oder Einübung. ${ }^{223}$ Zudem kann die Kognitivierungsphase auch ganz ausgelassen werden, wenn kein Bedürfnis nach expliziten Erklärungen besteht, weil die grammatischen Strukturen selbst erschlossen werden können ${ }^{224}$ oder schon bekannt sind. Es empfiehlt sich außerdem, der Präsentation einen kurzen Einstieg voranzustellen, welcher einen ersten Zugang zum Unterrichtsthema bietet und Schüler*innen dazu

\footnotetext{
${ }^{216}$ Vgl. Nieweler 2017, S. 195.

${ }^{217}$ Vgl. ebd.

218 Vgl. Plassmann 1992, S. 346.

${ }^{219}$ Vgl. Nieweler 2017, S. 195.

${ }^{220}$ Vgl. Plassmann 1992, S. 346.

${ }^{221}$ Vgl. Nieweler 2017, S. 195.

222 Vgl. Plassmann 1992, S. 346.

${ }^{223}$ Vgl. Nieweler 2017, S. 195.

224 Vgl. ebd.
} 
anregt, bereits vorhandenes Vokabular zu reaktivieren und sich sprachlich und mental auf die Präsentation einzustellen. ${ }^{225}$

\subsection{Aufbau}

Im Folgenden soll nun dargestellt werden, wie eine Unterrichtseinheit zum Thema soziale Medien im Arabischunterricht anhand der fünf Phasen nach Zimmermann, inklusive optionalem Einstieg, aufgebaut werden könnte.

\subsubsection{Einstieg}

Den Schüler*innen wird ein Bild gezeigt, auf dem die Logos von Twitter, Instagram und Facebook zu sehen sind. Daraufhin wirft die Lehrkraft mehrere Fragen in den Raum und erkundigt sich beispielsweise, ob die Schüler*innen diese Logos kennen, welche Assoziationen sie mit diesen Logos verbinden, inwiefern diese Logos und die dahinterstehenden Plattformen für sie persönlich von Bedeutung sind und welches Unterrichtsthema sich anhand dieser Einleitung ergeben könnte. Beispielhafte Antworten könnten das Thema Internet oder Kommunikation sein. Der Oberbegriff für soziale Medien - wasa $\bar{a}^{\prime} i l$ at-tawāṣul al-iğtimā ${ }^{c} \bar{l}-$ sollte ihnen bis hierher noch unbekannt sein.

\subsubsection{Präsentation}

Die Präsentation setzt sich aus zwei Teilen zusammen. Einerseits beinhaltet sie die ersten fünfzig Sekunden eines Videos der Sendung Shabab Talk ${ }^{226}$, welche von Deutsche Welle produziert und mittlerweile in das Format Jaafar Talk umgewandelt wurde. Shabab Talk wurde vom Moderator Jaafar Abdul Karim selbst als „Stimme der arabischen Jugend“ bezeichnet und widmete sich gesellschaftskritischen Themen wie Presse- und Meinungsfreiheit, Religion und Homosexualität. Allein in Ägypten hatte die Sendung zeitweise über vier Millionen Zuschauer*innen und war auch in anderen arabischsprachigen Ländern ausge-

${ }^{225} \mathrm{Vgl}$. Technische Universität Dresden 2017.

2015 جعفر توك Vgl. DW 
sprochen beliebt. ${ }^{227}$ In der ausgewählten Folge geht es darum, inwiefern junge Menschen in den Golfstaaten in den sozialen Medien ihre Meinung frei äußern können und wozu sie soziale Medien vorzugsweise verwenden. Obwohl nur die ersten fünfzig Sekunden des Videos in dieser Unterrichtseinheit angeschaut werden sollen, sind diese bereits sprachlich sehr anspruchsvoll und beinhalten viele neue Vokabeln und Ausdrücke. Der Videoausschnitt kann in dieser Phase mehrmals angeschaut werden und es kann gefragt werden, was die Schüler*innen schon verstanden haben und ob sie bereits bestimmte Ausdrücke gehört haben, die ihnen noch unbekannt sind. Diese können dann gemeinsam besprochen werden. Der zweite Teil der Präsentation ist das Transkript des Gesprochenen, welches die Lehrkraft anfertigt und an die Schüler*innen verteilt. Hierbei kann der unter dem Video stehende Text auf YouTube, welcher den Inhalt der Folge zusammenfasst, hilfreich sein, da er bereits den Großteil des Inhalts der ersten Minute beschreibt. Um diesen Schritt zu verdeutlichen, wurde ein solches Transkript erstellt, welches in Anhang I zu sehen ist. Das Transkript soll eigenständig von den Schüler*innen gelesen werden, damit sie im Anschluss gezielte Fragen stellen können. Es wird zusammen mit einer Liste neuer Vokabeln und ihrer deutschen Übersetzungen, welche in Anhang II zu sehen ist, präsentiert. Die neuen Vokabeln sind auch im Transkript hervorgehoben.

Idealerweise können die Schüler*innen sich den Inhalt des Transkripts, und somit des Videoausschnitts, unter Zuhilfenahme der Vokabelliste erschließen. Das Video kann auch noch mehrere Male gemeinsam mit dem Transkript angeschaut werden, wodurch sich auch die entsprechende Aussprache der neuen Worte festigen lässt. Darüber hinaus kann die Lehrkraft die neuen Vokabeln vorsprechen und die Schüler*innen individuell oder in der Gruppe auffordern, sie nachzusprechen. Auch einzelne Sätze des Transkripts könnten hier vorgelesen werden oder es kann direkt anhand des Sprachbeispiels im Video nachgesprochen werden. Idealerweise beginnen die Schüler*innen bereits in dieser Phase, sich näher mit der Verwendung sozialer Medien in den arabischsprachigen Ländern zu befassen und den Inhalt des Videoausschnitts in Bezug auf ihr eigenes Leben zu reflektieren. Etwaige Verständnisfragen werden in diesem Teil der Stunde geklärt.

227 Vgl. Sasse 2015. 
Die Präsentation verfolgt noch ein zweites Ziel, das über die Vorstellung neuer Ausdrücke hinausgeht: Da viele der im Video verwendeten und in der Vokabelliste übersetzen Ausdrücke bestimmte Genitivverbindungen sind, soll dieses grammatische Thema noch einmal aufgegriffen werden. Bei Schüler*innen, die sich auf Niveau B1 befinden, sollte das Thema auf jeden Fall schon einmal behandelt worden sein. Hier kommt die optionale Kognitivierung ins Spiel. Möglicherweise erinnern sich die Schüler*innen noch an den Aufbau der bestimmten Genitivverbindung, beispielsweise die korrekte Verwendung des bestimmten Artikels, und benötigen keine explizite Wiederholung. Besteht jedoch Wiederholungsbedarf, so kann die Lehrkraft jetzt noch einmal das Konzept der bestimmten Genitivverbindung erklären. Ein positiver Nebeneffekt dieses Videoausschnitts ist, dass er nicht nur grundsätzlich mehrere Genitivverbindungen enthält, sondern auch zwei Beispiele für eine dreiteilige Genitivverbindung - mawḍ̆ 'halqat al-yawm und hayāt šabāb al-hhalīg - sowie zwei Genitivverbindungen, an die Adjektive an-

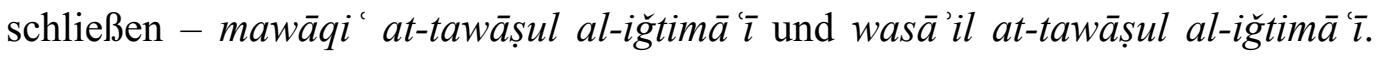
Folglich können diese konkreten Beispiele aus dem Transkript dazu dienen, das Thema mehrteiliger Genitvverbindungen und die Regeln für Adjektive, welche Teile einer Genitivverbindung beschreiben, zu wiederholen. Die Schüler*innen können hierzu im Transkript Markierungen und Annotationen vornehmen.

\subsubsection{Einübung}

Den Schüler*innen werden einfache Lückensätze präsentiert, in welche sie die neuen Vokabeln sinnvoll einsetzen müssen. Des Weiteren werden ihnen Wortgruppen vorgegeben, unter denen sie bestimmte Genitvverbindungen erkennen und beispielsweise zwischen einer Genitivverbindung und einer lediglich aus Substantiv und Adjektiv bestehenden Wortgruppe unterscheiden müssen. Hierbei soll sowohl die Verwendung der neuen Vokabeln in einem strukturierten Kontext geübt als auch das Thema Genitivverbindung gefestigt werden. Die Sätze und Wortgruppen werden von der Lehrkraft erstellt und beinhalten, abgesehen von den neuen Ausdrücken, die eingefügt werden müssen, lediglich bereits bekanntes Vokabular, um sicherzustellen, dass die Schüler*innen die Aufgabe bewältigen können. Die für diese Unterrichtseinheit erstellten Lückensätze und Wortgruppen sind jeweils in 
Anhang III und Anhang IV zu sehen. Die Lückensätze können in zukünftigen Praxisversuchen je nach Kenntnisstand der Schüler*innen angepasst werden, solange das Ziel, neue Ausdrücke sinnvoll einzufügen, erhalten bleibt. Das gleiche Prinzip gilt für die Wortgruppen zur Festigung der bestimmten Genitivverbindung. Beide Aufgaben werden jeweils eigenständig erledigt und dann in der Gruppe verglichen, wobei die Lehrkraft sicherstellt, dass alle Arbeitsblätter korrekt ausgefüllt sind.

\subsubsection{Transfer}

Auch der Transfer beinhaltet zwei unterschiedliche Aufgaben, um sowohl das Wissen über die Genitivverbindung als auch die neuen Vokabeln anzuwenden. In der ersten Aufgabe werden die Schüler*innen in Gruppen von zwei bis drei Personen aufgeteilt. Jede Gruppe erhält einen Stapel Karten, auf denen Substantive stehen und einen Stapel mit Adjektiven. Ein Beispielkartensatz ist in Anhang V zu sehen. Die Karten umfassen sowohl neue Wörter aus dem Text als auch bereits bekannte Vokabeln. Diese sollen sie individuell zusammenfügen, um fünf Genitivverbindungen mit zwei oder drei Teilen zu bilden und jeweils ein sinnvolles Adjektiv hinzuzufügen. Die Wörter werden ohne den bestimmten Artikel präsentiert, welcher selbst an gegebener Stelle von den Schüler*innen beigefügt werden muss. Die Lehrkraft ist während dieser Aktivität ansprechbar, lässt die Schüler*innen aber weitestgehend eigenständig arbeiten. Die Gruppen stellen ihren Mitschüler*innen ihre Ergebnisse anschließend vor, indem sie die Karten in der richtigen Reihenfolge mit Magneten an der Tafel befestigen und den bestimmten Artikel dazuschreiben. Danach versuchen die Schüler*innen der anderen Gruppen, die vorgestellten Beispiele in mündlichen Sätzen zu verwenden.

In der zweiten Transferübung sollen die neuen Vokabeln gefestigt werden. Zudem soll hier verstärkt der Aspekt der kritischen Auseinandersetzung mit den sozialen Medien eingebracht werden, weshalb die Übung auf einem Text zu den Vor- und Nachteilen der Benutzung sozialer Medien basiert. Hierzu könnte sich die Lehrkraft einen eigenen Text ausdenken, oder aber einen bereits vorhandenen Text umschreiben und anpassen. Auf der Internetseite mawdoo3 findet sich beispiels- 
weise ein $A_{r t i k e l}{ }^{228}$, der viele positive und negative Effekte sozialer Medien auf das Individuum und die Gesellschaft anspricht. Der Artikel ist lang und für diese Übung sollten je nach Kenntnisstand der Schüler*innen und verfügbarer Zeit nur zwei bis fünf Abschnitte übernommen werden. Dabei empfiehlt es sich, die Stellen auszuwählen, deren Informationen am relevantesten für Schüler*innen der Sekundarstufe sind. Beispielsweise gibt es Abschnitte, die den Nutzen sozialer Medien für Unternehmer*innen erläutern, was mit der Lebenswirklichkeit jugendlicher Schüler*innen vermutlich wenig zu tun hat. Der Text wurde für diese Unterrichtseinheit insofern umgearbeitet, als dass schwierige und unbekannte Wörter durch leichtere ersetzt wurden. Zudem wurden dort Lücken gelassen, wo die neuen Vokabeln sinnvoll hineinpassen würden. Die Schüler*innen sollen nun in Eigenarbeit den Lückentext mit den neuen Vokabeln aus dieser Stunde ausfüllen. Hier sollte angestrebt werden, dass ihnen keines der Wörter unbekannt ist und sie den Inhalt der Absätze selbstständig erschließen können. Der Text, der für diese Unterrichtseinheit erstellt wurde, ist in Anhang VI zu sehen.

\subsubsection{Anwendung}

Die Anwendungsphase ist die kreativste der fünf Unterrichtsphasen, in welcher die Schüler*innen ihr erworbenes Wissen ohne jegliche Anleitung der Lehrkraft einsetzen. Die Anwendung wird häufig in der Hausaufgabe und nicht in der tatsächlichen Unterrichtsstunde durchgeführt. Für die hier vorgeschlagene Unterrichtseinheit wäre die Hausaufgabe, dass Schüler*innen einen Text verfassen, in dem sie ihre eigene Benutzung sozialer Medien reflektieren. Dieser Text soll beinhalten, welche sozialen Medien sie vorrangig verwenden und $\mathrm{zu}$ welchen Zwecken. Außerdem sollen sie bestimmte Vor- und Nachteile sozialer Medien in ihrem Leben erläutern, wobei sie sich auf die im vorangegangen Lückentext erwähnten Beispiele beziehen können. Die Schüler*innen sollen dazu angehalten werden, im Text so viele der neuen Vokabeln und Genitivverbindungen wie möglich zu verwenden.

${ }^{228}$ Vgl. Mawdoo3 2020. 


\subsection{Mögliche Modifizierungen}

Wie zu Beginn erwähnt, könnte diese Unterrichtseinheit auch über mehrere Stunden hinweg durchgeführt werden. Je mehr Zeit dafür verfügbar ist, desto mehr können die individuellen Phasen ausgeweitet und verstärkt werden. Eine andere mögliche Anwendung wäre zum Beispiel, dass die Schüler*innen die Vor- und Nachteile sozialer Medien aus dem Lückentext und aus ihrer Hausaufgabe dazu verwenden, im Unterricht eine Debatte über die sozialen Medien zu führen, wobei eine Klassenhälfte ihre Verwendung befürwortet und die andere dagegen hält. Zudem könnten die Schüler*innen untereinander Interviews durchführen, in denen sie sich gegenseitig nach ihrer individuellen Einstellung zu und Verwendung von sozialen Medien befragen. Es ist auch vorstellbar, dass diese Unterrichtseinheit nur ein Teil eines größeren, übergeordneten Themenbereichs ist und noch weitere Unterrichtsstunden zu den sozialen Medien folgen. In anschließenden Stunden könnten beispielsweise individuelle Plattformen wie Facebook, Twitter und Instagram auf Arabisch behandelt werden, wobei man Posts oder Kommentare analysieren könnte. Dies könnte weiterhin zur Diskussion von Netiquette führen und Kommentare und Posts könnten gleichzeitig als Basis für die Erforschung der arabischen Sprache im Netz dienen. Wie Grünewald hervorhebt, unterscheidet sich die Sprache der sozialen Medien oft von der Standardsprache. Sich im Fremdsprachenunterricht gezielt mit den soziolinguistischen Besonderheiten der netzspezifischen Kommunikation auseinanderzusetzen kann deshalb interessant und förderlich sein. ${ }^{229}$ Zudem könnten weitere Zeitungsartikel oder Expertenvideos zu den Chancen und Gefahren sozialer Medien auf Arabisch folgen und den Schüler*innen könnte aufgetragen werden, ihre eigene Sensibilisierungskampagne zum Thema soziale Medien auf Arabisch zu erstellen.

Darüber hinaus bietet sich die Möglichkeit, den Aspekt der Meinungsfreiheit, welcher im Video erwähnt wird und eine wichtige neue Vokabel darstellt, näher zu besprechen. Was ist Meinungsfreiheit? Ist die Präsenz oder Abwesenheit von Meinungsfreiheit in den sozialen Medien für deutsche Schüler*innen relevant? Warum ist sie für junge Menschen in den arabischen Ländern ein brisantes Thema? Diese und andere Fragen könnten neue, sprachlich komplexe Diskussionen in der

${ }^{229}$ Vgl. Grünewald 2016, S. 465. 
Klasse anregen und der Lehrkraft eine weitere Möglichkeit bieten, gesellschaftsrelevante Themen zu besprechen. Eine zusätzliche Aktivität, die sich in diesem Themenbereich anbietet, ist die nähere Erforschung der Verschriftung englischer Wörter auf Arabisch. Einerseits werden die Schüler*innen in der hier vorgestellten Unterrichtseinheit mit den arabischen Entsprechungen für Twitter, Facebook, Instagram und YouTube bekannt gemacht und andererseits ist bei näherer Betrachtung der arabischen Internetsprache zu erwarten, dass noch weitere solcher Lehnwörter zu finden sind. Obwohl dieser Ansatz banal erscheinen mag, ist zu bedenken, dass das Erkennen von Lehnwörtern im Arabischen für Nicht-Muttersprachler*innen tatsächlich oft sehr schwierig ist ${ }^{230}$ und eine wichtige sprachliche Übung darstellen kann.

Das Video von Shabab Talk wurde für diese Unterrichtseinheit gezielt gewählt, da die Sendung selbst aufklärerische Ziele verfolgt und aktuelle, kontroverse Themen, welche vor allem die arabischsprachige Jugend beschäftigen, multiperspektivisch darstellt. Die Sendung eignet sich demnach auch gut als arabischsprachiges Medium für die eigenständige Verwendung außerhalb des Unterrichts. Die Lehrkraft könnte diesen Aspekt im Unterricht verdeutlichen und als Beispiel nehmen, um Schüler*innen auf der Suche nach altersgerechten und gehaltvollen medialen Inhalten zu unterstützen.

Die Unterrichtsstunde versucht innerhalb verschiedener Arbeitsaufträge, die Kompetenzen Hören, Lesen, Sprechen und Schreiben zu fördern und zudem Grammatikkenntnisse zu festigen. Es wäre aber auch denkbar, in einer Stunde nur das Thema der neuen Vokabeln aufzugreifen und die entsprechenden Übungen zu durchlaufen und in der nächsten Stunde die Grammatik der bestimmten Genitvverbindung zu vertiefen. Die Arbeitsaufträge beinhalten Möglichkeiten für Einzelund Partnerarbeit sowie die Diskussion im Klassenverband. Die verwendeten Materialien umfassen sowohl digitale Medien als auch Materialien wie Stift, Papier und Tafel. Lernziel soll zum einen sein, neue Vokabeln aufzunehmen und zu verwenden sowie bestehende Kenntnisse der bestimmten Genitivverbindung auszubauen. Andererseits sollen die Schüler*innen auch Wissen über die Verwendungszwecke und die Vor- und Nachteile sozialer Medien erhalten, dies auf ihr

${ }^{230}$ Vgl. Van Mol 2006, S. 306. 
eigenes Leben beziehen und ihren eigenen Konsum sozialer Medien kritisch reflektieren. Dies soll unter anderem dazu beitragen, die Vielfalt der digitalen Medienlandschaft näher kennenzulernen und die Nutzung privater Internetdienste differenzierter zu betrachten. ${ }^{231}$

\subsection{Einschätzung}

Eine Schwierigkeit beim Entwurf dieser theoretischen Unterrichtseinheit war, sprachliche Inhalte mit Inhalten zur Medienbildung und Mediennutzung zu verbinden. Die Versuchung ist groß, Aspekte der kritischen Medienbildung zum Hauptthema des Unterrichts $\mathrm{zu}$ machen, ohne dabei konkretes und nützliches sprachliches Wissen zu vermitteln. Andererseits ist es leicht, die Unterrichtsstunde auf Basis der Vokabeln und grammatischen Strukturen, die vermittelt werden sollen, zu planen und dabei jegliche Aspekte von Medienbildung oder Ausbildung von Medienkompetenz zu ignorieren. Gerade im Arabischunterricht, der aufgrund der linguistischen und kulturellen Distanz der arabischen Sprache zum Deutschen von Vornherein mehr Zeit in Anspruch nimmt als der Unterricht anderer europäischer Sprachen ${ }^{232}$, ist die Gefahr groß, sich allein an den sprachlichen Zielen, die innerhalb des Schuljahres erreicht werden sollen, zu orientieren und keine Verantwortung für die Ausbildung konkreter Medienkompetenzen oder für die Vermittlung medienpädagogischen Wissens zu übernehmen. Dies bestärkt auch die Forderung, Medienbildung grundsätzlich als Auftrag des Arabischunterrichts zu erkennen und im Lehrplan zu verankern, damit diese Zielsetzung nicht verloren geht und kontinuierlich in verschiedenen Unterrichtsstunden umgesetzt werden kann. Die Erwartungshaltung sollte auf keinen Fall sein, dass jede Unterrichtsstunde sowohl sprachliche als auch medienpädagogische Ziele verfolgt. Vielmehr sollte sichergestellt werden, dass sich Medienbildung regelmäßig im Unterricht wiederfindet und neue Medien oft und sinnstiftend im Unterricht verwendet werden.

\footnotetext{
${ }^{231}$ Vgl. Ständige Konferenz der Kultusminister der Länder in der Bundesrepublik Deutschland 2016.

${ }^{232}$ Vgl. Guder 2005, S. 66.
} 
Es wird deutlich, dass jede Phase der vorgestellten Unterrichtsstunde geändert werden könnte, dass die konkrete Vermittlung medienspezifischen Wissens auf verschiedene Arten und Weisen geschehen kann, dass je nach Zeitrahmen, Bedürfnissen und Interessen andere Schwerpunkte gesetzt werden können und dass Schüler*innen die vermittelten Inhalte unterschiedlich reflektieren und anwenden werden. Der präsentierte Vorschlag ist also nur ein mögliches Beispiel für die Verbindung des Lernens mit und über Medien im Arabischunterricht, der vor allem dazu dient, zu verdeutlichen, dass diese Verbindung möglich und sinnvoll ist. 


\section{Fazit}

Die Förderung von Medienbildung im schulischen Arabischunterricht beginnt damit, zwischen den Begriffen Medienbildung und Medienkompetenz zu unterscheiden und zu erkennen, dass beide im Unterricht Beachtung finden müssen. Medienbildung ist das pädagogische Auseinandersetzen mit Medien und beschreibt einen konkreten Bildungsauftrag, der sich an die Lehrkraft wendet. Ziel der Medienbildung soll es sein, Schüler*innen Medienkompetenzen zu vermitteln, also konkrete Fähigkeiten, die sie zum verantwortungsvollen, zielgerichteten und bedürfnisbezogenen Umgang mit neuen Medien befähigen. Dementsprechend muss der Unterricht sowohl das Lernen über Medien als auch das Lernen mit ihnen umfassen. Medienbildung als schulischer Pflichtauftrag soll nicht nur in ausgewählten Schulfächern umgesetzt werden, sondern muss in jedem Fachlehrplan verankert sein und entsprechend in jedem Unterrichtsfach verwirklicht werden. Folglich müssen auch die fremdsprachlichen Fächer - und somit der Arabischunterricht - dieser Forderung nachkommen und fachspezifische Möglichkeiten schaffen, Schüler*innen zur kritischen Betrachtung neuer Medien anzuleiten und sie im kompetenten Umgang mit diesen Medien zu unterstützen. Hierbei ist essenziell, dass Medienbildung dem Primat des Pädagogischen folgt und zuerst die pädagogische Zielsetzung definiert werden muss, um neue Medien dann als Hilfsmittel in der Verwirklichung dieser Ziele einzusetzen. Medienverwendung ist nie Selbstzweck.

Bei der Umsetzung medienpädagogischer Ziele fällt der Lehrkraft eine entscheidende Rolle zu, da sie die Medienerfahrungen ihrer Schüler*innen verstehen und daran anknüpfen, Lernenden bei der Bewertung und Analyse verschiedener Medien helfen, positive und negative Effekte neuer Medien veranschaulichen und Schüler*innen in der kreativen Verwendung dieser Medien anleiten soll. Die Lehrkraft muss folglich nicht nur allgemeine Medienkenntnisse besitzen, sondern auch über fachspezifische Medienkompetenz verfügen. Der Erwerb dieser geforderten Kenntnisse soll idealerweise als Teil des Lehramtsstudiums stattfinden und auch für bereits aktive Lehrkräfte durch Fort- und Weiterbildungen stetig gefördert werden. Da es für Arabischlehrer*innen in Deutschland weder einen Lehramtsstudiengang noch formalisierte Fort- und Weiterbildungsangebote gibt, ist anzu- 
nehmen, dass bereits tätige Arabischlehrkräfte über eingeschränkte Kenntnisse im Bereich Medienbildung verfügen, was Lehrer*innen und Schüler*innen gleichermaßen vor große Herausforderungen stellt. Für die entfernte Zukunft könnte die institutionelle Verankerung der Arabischdidaktik und ein entsprechendes Lehramtsstudium diese Defizite ausgleichen. Für den Moment sollte jedoch weiter in die Vernetzung von Arabischlehrer*innen in Deutschland investiert und bereits existente Initiativen für Online-Angebote im Bereich Lehrerbildung ausgebaut werden.

Die Analyse der Arabischlehrpläne zeigt, dass Medienbildung und die Ausbildung von Medienkompetenzen auf verschiedene Weise in den Lehrplänen verankert sind. In einigen Lehrplänen werden konkrete neue Medien erwähnt, die innerhalb des Unterrichts Verwendung finden sollen. In anderen wird lediglich von multimedialen Texten gesprochen, wobei nicht weiter erläutert wird, welche Medien hier zum Einsatz kommen. In manchen Lehrplänen ist zudem eine deutliche Einbettung digitaler Medien in bestimmte Themengebiete zu erkennen und angestrebte Kompetenzziele in den Bereichen Lesen, Hören, Sprechen und Schreiben sollen bewusst durch den Einsatz neuer Medien unterstützt werden. Des Weiteren wird in den Lehrplänen oft vom Einsatz neuer Medien zur Bereitstellung von authentischen Materialien gesprochen. Jedoch wird nicht immer genau erläutert, um welche Materialien es sich handelt und es bleibt zu vermuten, dass dies von Internetseiten über Nachrichtenbeiträge bis Fernsehserien alles einschließen kann.

Es zeigt sich jedoch auch deutlich, dass viele Lehrpläne den Einsatz neuer Medien vor allem als nutzbringend für die Vermittlung kulturellen Wissens betrachten und dass digitale Angebote wie Chatforen dazu beitragen können, Schüler*innen den direkten Austausch mit Arabischmuttersprachler*innen zu ermöglichen. Außerdem wird mehrmals hervorgehoben, dass digitale Medien den Schüler*innen bei der eigenständigen Recherche über die arabischsprachigen Länder behilflich sein können und zudem zur Ausbildung besseren Hörverstehens und der Vermittlung der arabischen Dialekte beitragen. Der Lehrplan aus Australien hebt sich im Vergleich deutlich ab, denn er beinhaltet als einziger Lehrplan explizite Bezüge zum Thema Technikethik und erwartet beispielsweise, dass die Schüler*innen im Arabischunterricht lernen, wie Kulturkreise in neuen Medien moralisch vertretbar dargestellt werden können. Der Vergleich der Lehrpläne mit den von der KMK 
formulierten Kompetenzen in der digitalen Welt verdeutlicht, dass die Lehrpläne die Vermittlung bestimmter Kompetenzen bereits anstreben. Grundsätzlich ist jedoch auch zu beobachten, dass die meisten Lehrpläne mehr Wert auf die Verwendung neuer Medien als auf deren kritische Betrachtung legen. Hier wird das Lernen mit Medien deutlich priorisiert und dem Lernen über Medien wird zu wenig Bedeutung beigemessen.

Die präsentierten Anwendungsbeispiele weisen deutliche Gemeinsamkeiten mit den Vorschlägen in den Lehrplänen auf. Auch hier zeigt sich, dass vor allem eigenständige Recherche, beispielsweise in Form von WebQuests, durch digitale Medien unterstützt werden kann. Außerdem verweisen mehrere der genannten Beispiele auf die Bereitstellung authentischer Materialien durch neue Medien, welche zum Erwerb soziokultureller Kenntnisse beitragen und den Lernenden beispielsweise durch authentische Videos Einblicke in den Alltag der arabischsprachigen Länder gewähren sollen. Zudem bieten authentische Materialien vielfältige Möglichkeiten, um Lernende auch mit den sprachlichen Varietäten der arabischen Welt vertraut zu machen und beispielsweise Hörmaterial in verschiedenen Dialekten bereitzustellen. Darüber hinaus können durch das Internet auch Videos und Hörtexte verfügbar gemacht werden, in denen Arabischmuttersprachler*innen Hocharabisch und Umgangssprache parallel verwenden. Dies kann den Lernenden helfen, größere Sprachbewusstheit auszubilden und zu erkennen, welche soziolinguistischen Situationen welche Varietät verlangen. Zudem können vor allem die sozialen Medien Möglichkeiten bieten, Lernende auch an verschriftete Umgangssprache heranzuführen.

Hinzu kommt, dass Lehrkräfte durch digitale Medien eigene Bildungsmedien und Materialien für den Unterricht erstellen können. Dies beinhaltet beispielsweise Internetseiten, welche durch Videos, Hör- und Lesetexte, kulturelle Informationen und interaktive Aktivitäten multimediale Lernumgebungen bieten, die mehrere Kompetenzbereiche unterstützen können. Vor allem interaktive Videos stellen hier einen großen Mehrwert dar, da sie es den Lernenden ermöglichen, ihre sprachlichen Mittel auch außerhalb des Unterrichts in kommunikativen Situationen zu festigen. Dies schafft auch einige logistische Vorteile, da Rückmeldungen nicht ausschließlich im Unterricht gegeben werden müssen, sondern beispielsweise durch virtuelle Interaktionen bereitgestellt werden. Zuletzt können neue Medien 
auch zur Vernetzung Lernender in sogenannten communities of practice beitragen und den Austausch zwischen Lernenden und Muttersprachler*innen fördern, beispielsweise durch Sofortnachrichtendienste und Chatforen.

Laut KMK spielt die Lehrkraft vor allem bei der Auswahl gehaltvoller, altersgerechter und pädagogisch wertvoller Medien eine wichtige Rolle. Diese Auswahl sollte nach Möglichkeit unter Zuhilfenahme bestimmter Kriterien geschehen. In der Analyse verfügbarer Medien für den Arabischunterricht laut Kriterien der KMK und des vzbv sticht Duolingo als Negativbeispiel heraus. Die einzigen positiven Aspekte dieser Lernapp sind die spielerische Gestaltung, welche vor allem jüngeren Schüler*innen gefallen könnte und die Vermittlung alltagsrelevanter Vokabeln. Leider finden sich in der App aber zahlreiche Beispiele für eine fehlerhafte Aussprache und Mischung von hocharabischen und umgangssprachlichen Elementen ohne nähere Erklärung. Im Vergleich dazu ist InterLingo eine angemessene Internetseite, welche Lehrer*innen und Schüler*innen nützen kann. InterLingo zeichnet sich durch ihre gute Strukturierung aus und präsentiert Übungen für die Ausbildung der vier Kompetenzen - Hören, Lesen, Sprechen, Schreiben. Trotzdem sind einige Inhalte für Schüler*innen ohne gute Englischkenntnisse nicht zugänglich und bräuchten viel Anleitung und Anpassung durch die Lehrkraft.

Die Internetseite des Defense Language Institute Foreign Language Center zeichnet sich vorrangig durch die Fülle an Materialien in den arabischen Dialekten aus, wobei die Accents Library beispielsweise Hörtexte auf Hocharabisch und in zehn weiteren Dialekten bereitstellt. Diese Materialien könnten vor allem für vergleichende Übungen im Unterricht nützlich sein. Die Inhalte richten sich jedoch vorrangig an das US-Militär, weshalb sie oftmals für den schulischen Unterricht irrelevant sind. Zudem sind die meisten Übungen nur für fortgeschrittene Lernende geeignet. Auch Lingualism eignet sich vor allem als Quelle für vielfältige Materialien in den arabischen Dialekten. Im Vergleich zum DLIFLC sind die Themen, die in den Büchern und in den Hörtexten behandelt werden, für Schüler*innen relevant und vor allem die vokalisierten Transkripte für die Hörtexte können zur Ausbildung von differenziertem Hörverstehen beitragen. Des Weiteren fungiert die Lingualism-Internetseite als ein Beispiel für communities of practice, in denen sich Arabischlernende austauschen können und Zugang zu Ressourcen für eigenständiges Lernen bekommen. Jedoch benötigen die Schüler*innen gute bis sehr 
gute Englischkenntnisse und viel Eigeninitiative, um die verfügbaren Ressourcen tatsächlich sinnvoll zu nutzen.

Die Internetseite Learning Arabic von Al Jazeera sticht hauptsächlich durch eine große Auswahl an Themen auf differenzierten Sprachniveaus hervor und stellt viele abwechslungsreiche Übungen für Arabischlernende bereit. Dadurch können vor allem Schreiben, Hören und Lesen gefördert werden. Die angebotenen Inhalte sind jedoch fast ausschließlich auf Hocharabisch verfügbar und da jegliche Hörund Lesetexte von Al Jazeera produziert sind, kann man hier nicht von einem neutralen Medium sprechen. Im Vergleich zu Learning Arabic ist Playaling viel simpler aufgebaut, kann aber dennoch ein nützliches Medium für den Arabischunterricht darstellen. Vor allem für die Ausbildung von Hörverstehen, sowohl in den Dialekten als auch auf Hocharabisch, eignen sich die Videos inklusive interaktiver Transkripte sehr gut. Die Anmerkungen in den Transkripten können zudem beispielsweise eigenständige Wörterbucharbeit unterstützen. Auch der gut strukturierte Aufbau, die Abwesenheit jeglicher Werbung und die vielfältige Themenauswahl machen Playaling zu einer geeigneten Ressource für individuelles Lernen im schulischen Kontext. Um die Internetseite, und vor allem die grammatischen Anmerkungen in vollem Rahmen nutzen zu können, sind jedoch gute Englischkenntnisse vonnöten.

Unter den analysierten Bildungsmedien sticht vor allem Khallina positiv hervor. Die Internetseite bietet fünfzehn umfangreiche Module zu verschiedenen kulturellen und sprachlichen Themen. Jedes Modul beinhaltet abwechslungsreiche Arbeitsaufträge und eine Vielfalt an authentischen Materialien auf Hocharabisch und Umgangssprache. Zudem wird deutlich, dass Khallina versucht, kontroverse Themen multiperspektivisch darzustellen und sie Schüler*innen zugänglich zu machen. Der Aufbau der Module zeigt eine nachvollziehbare Progression von Präsentation zu Anwendung, bietet aber oft zu wenige Gelegenheiten, neue Inhalte hinreichend einzuüben.

Der Vergleich existenter digitaler Medien zeigt, dass vor allem die Kompetenzbereiche Hören und Lesen vom Einsatz neuer Medien profitieren können. Besonders die Kombination von Hörtexten und Transkripten eröffnet vielfältige Möglichkeiten, differenzierteres Hörverstehen unter Lernenden auszubilden. Des 
Weiteren können digitale Medien Materialien in den arabischen Dialekten bereitstellen, und vor allem Videos, in denen mehrere Sprachregister verwendet werden, können zur Ausbildung größerer Sprachbewusstheit beitragen. Zuletzt verdeutlichen Internetseiten wie Learning Arabic, Playaling und Khallina auch, dass neue Medien wichtige Einblicke in die arabischsprachigen Gesellschaften gewähren und für Schüler*innen relevante Themenbereiche ansprechen können.

Als Antwort auf die anfangs gestellte Forschungsfrage ist festzuhalten, dass, wie in den Lehrplänen und Anwendungsbeispielen zu sehen, die Ausbildung konkreter Medienkompetenzen bereits auf vielfältige Weise im Arabischunterricht stattfindet, dem kritischen Umgang und der reflektierten Auseinandersetzung mit der modernen Medienlandschaft jedoch kaum Beachtung geschenkt wird. In Bezug auf die Frage, ob und wie man neue Medien sinnvoll im Arabischunterricht einsetzen kann, präsentiert diese Forschungsarbeit einige Anwendungsvorschläge, die zur Ausbildung der Kompetenzen Lesen, Hören, Sprechen und Schreiben beitragen und zudem Sprachbewusstheit und vertieftes Wissen über die Kulturen und Gesellschaften der arabischsprachigen Welt fördern. Um Medienbildung im schulischen Arabischunterricht auszubauen, bedarf es jedoch eines verstärkten Fokus auf das Lernen über Medien seitens der Lehrkräfte und Schüler*innen. Um diese Herausforderung anzugehen, legt diese Forschungsarbeit eine konkrete Unterrichtseinheit vor, welche die medienpädagogischen Ziele des Lernens mit und über Medien im fremdsprachlichen Arabischunterricht vereint.

Wie in der Einleitung erwähnt, stellt die vorliegende Forschungsarbeit einen ersten Versuch dar, die konkreten Ziele der schulischen Medienbildung mit den angestrebten Zielen und Kompetenzen des fremdsprachlichen Arabischunterrichts zu verbinden. Da es kaum Forschung zum schulischen Arabischunterricht in Deutschland gibt, bedeuten die präsentierten Erkenntnisse einen wichtigen Schritt in der wissenschaftlichen Auseinandersetzung mit dem Feld der Arabischdidaktik. Die vorliegende Forschungsarbeit konnte feststellen, dass Medienbildung im Arabischunterricht nicht optional, sondern, zumindest laut Empfehlungen der KMK, verpflichtend ist. Das größere Ziel sollte sein, den schulischen Arabischunterricht in Deutschland weiter auszubauen, was unter anderem die Erstellung formalisierter Lehrpläne beinhaltet. Die hier dargestellten Möglichkeiten, Medienbildung im Lehrplan zu verankern und neue Medien im Unterricht einzusetzen, könnten 
maßgeblich zur Entwicklung eines zukünftigen Arabischlehrplans beitragen, welcher die Vorgaben der KMK für schulische Bildung, unter anderem auch Medienbildung, erfüllt. Somit versucht diese Forschungsarbeit, einen Beitrag zur Förderung des fremdsprachlichen Arabischunterrichts an deutschen Schulen zu leisten.

Im Rahmen dieser Forschungsarbeit war es nicht möglich, umfangreiche Informationen zu bereits existenten Arabischprogrammen an deutschen Schulen zu präsentieren. Auch konnte nicht näher auf den Bildungshintergrund der in Deutschland tätigen Arabischlehrer*innen eingegangen werden. Zudem wurden lediglich drei Lehrpläne aus Europa analysiert und fast ausschließlich Anwendungsbeispiele aus den USA vorgestellt. Zukünftige Forschungen in diesem Bereich sollten umfangreiche Studien zum Arabischunterricht in Deutschland und den Qualifikationen der tätigen Arabischlehrkräfte durchführen, um ein ganzheitliches Bild zu schaffen. Zudem wäre es empfehlenswert, auch den Arabischunterricht in verschiedenen europäischen Ländern näher zu erforschen und die Suche nach Arabischlehrplänen in Europa auszuweiten. Letztlich wäre es interessant, die im Rahmen dieser Arbeit entwickelte Unterrichtseinheit in der Praxis umzusetzen und zu sehen, inwiefern sie tatsächlich zur Förderung von Medienbildung beitragen kann.

Einer Disziplin wie der Arabischdidaktik, die bisher ein eher marginales Dasein fristet, wäre es in jedem Falle zuträglich, die Vorgaben der KMK so gut wie möglich umzusetzen und ein Umfeld zu schaffen, in dem Arabischlehrer*innen vernetzt sind und gemeinsam medienpädagogische Ziele verfolgen. Dies würde nicht nur dazu beitragen, die Arabischdidaktik als ernst zu nehmende Disziplin zu etablieren, sondern auch dafür sorgen, dass von Anfang an ein Maß an Leistungsverantwortung und Vereinheitlichung geschaffen wird, an dem sich Arabischlehrer*innen jeglicher Schulen und Bundesländer orientieren können. Darüber hinaus hilft ein abgestimmtes Konzept in Bezug auf Medienbildung auch dabei, zukünftige Fortschritte in diesem Bereich messen und vergleichen zu können.

Arabischlehrer*innen sollten die stetig zu beobachtenden technologischen Veränderungen in unserer Welt nicht ignorieren. Stattdessen sollten sie diese als real anerkennen und sich offen für technologische Neuerungen zeigen und sich auf die neuen Möglichkeiten, die sich aus dieser Realität für den Arabischunterricht 
ergeben, einlassen. Die Arabischdidaktik ist gerade erst dabei in Deutschland Fuß zu fassen. Auf der einen Seite könnte man das kleine Netzwerk an Arabischlehrer*innen im deutschsprachigen Raum und die vergleichsweise überschaubare Menge an Ressourcen für den Arabischunterricht als Hindernis wahrnehmen und schlussfolgern, dass Arabischlehrkräfte in Deutschland viel länger brauchen werden, um innovative Lehrmethoden und Aktivitäten für zielgerichteten Medieneinsatz im Arabischunterricht zu entwickeln. Auf der anderen Seite könnte man aber auch die einzigartige Chance sehen, die sich dadurch ergibt, dass großes Interesse an der arabischen Sprache besteht und erhebliches unausgeschöpftes Potenzial im Bereich Arabisch als Fremdsprache existiert. Wenn sich Arabischlehrkräfte nicht nur zögerlich und gezwungenermaßen auf die Verwendung neuer Medien im Unterricht einlassen, sondern Medienbildung und Medienkompetenz als wesentlichen Bestandteil des Unterrichts anerkennen und konsequent in die Unterrichtsplanung und -durchführung einbeziehen, dann wird man sich zukünftig nicht mehr nur an Beispielen aus dem Ausland orientieren, sondern konkrete Erfahrungen aus dem deutschsprachigen Raum in aktuelle Diskussionen einbeziehen können. Arabischlehrer*innen in Deutschland bietet sich also die einmalige Chance und wichtige Verantwortung, innovative Anwendungsbeispiele, welche das Lernen $\ddot{u} b e r$ und mit Medien umfassen, zu konzipieren und gleichzeitig dringend nötige empirische Einblicke in die Arabischdidaktik im schulischen, außeruniversitären Kontext zu gewähren. 



\section{Anhang}

Anhang I. Transkript für die Präsentationsphase der theoretischen Unterrichtseinheit

أهلاً وسهلاً بكم في شباب توك من العاصمة الألمانية برلين، موضوع حلقة اليوم شباب الخليج وحرية التعبير على مواقع التواصل الاجتماعي. تويتر، فيسبوك،

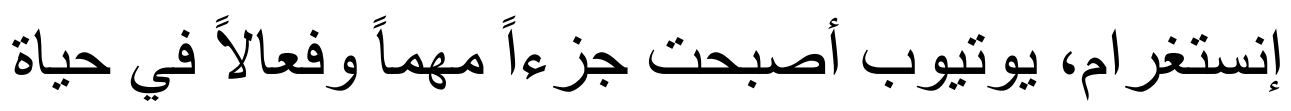
شباب الخليج الذي يمثل • ؟\% من المجتمع. هذه الثريحة النشيطة في المجتمع تعتمد اعتماداً كلياً على وسائل التواصل الاجتماعي في جميع نشاطاتها اليومية، (مثلاً)

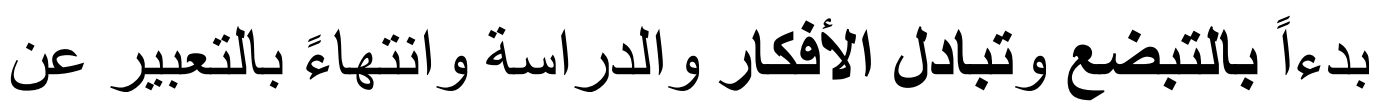

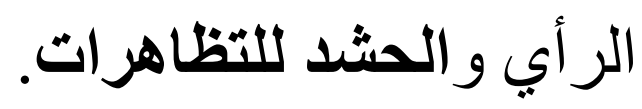


Anhang II. Vokabelliste für die Präsentationsphase der theoretischen Unterrichtseinheit

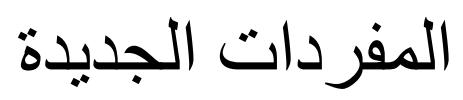

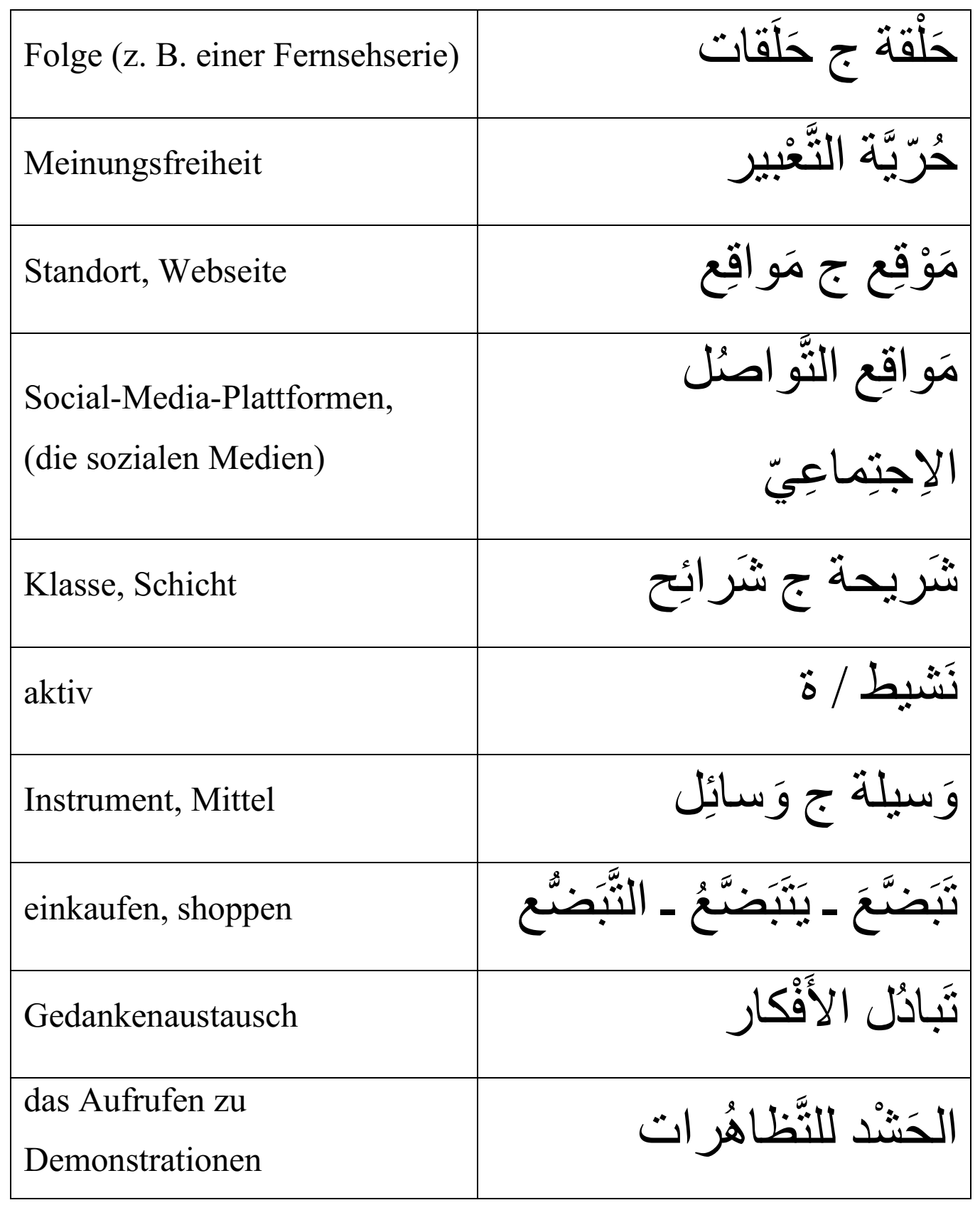


Anhang III. Lückensätze für die Einübungsphase der theoretischen Unterrichtseinheit

\section{املؤوا الفراغات بالمفردات الجديدة}

بالرغم من أنها كبيرة في السن، جدتي ماز الت

جداً.

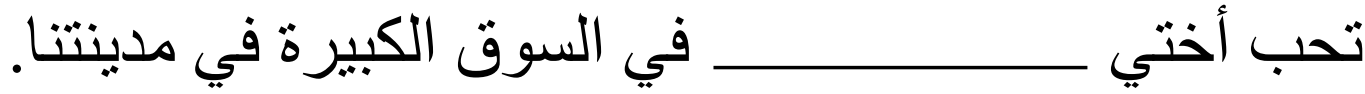

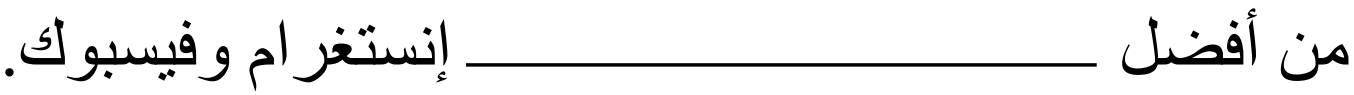

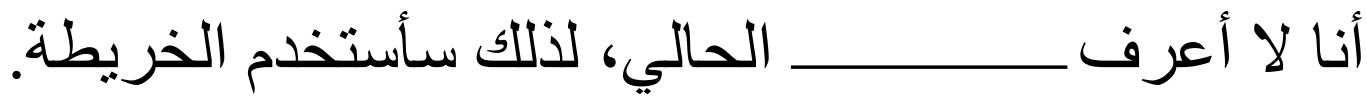

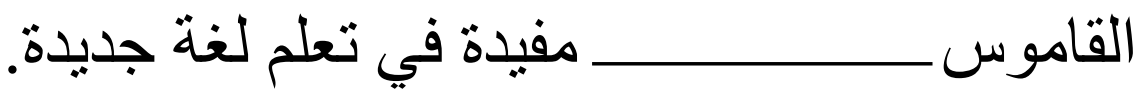

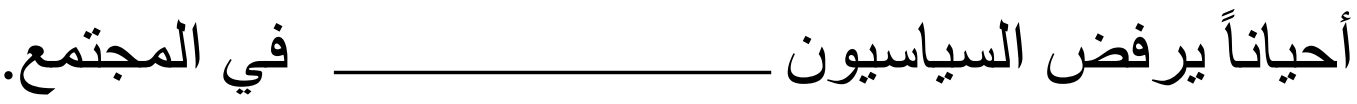

أتكلم أنا و أصدقائي عن كل شيء ونستمتع بـ

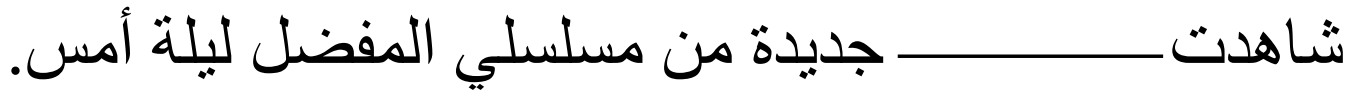

تعني أن كل شخص يمكنه أن يقول ما يريد.

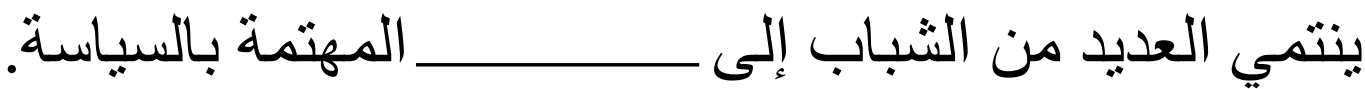


Anhang IV. Wortgruppen für die Einübungsphase der theoretischen Unterrichtseinheit

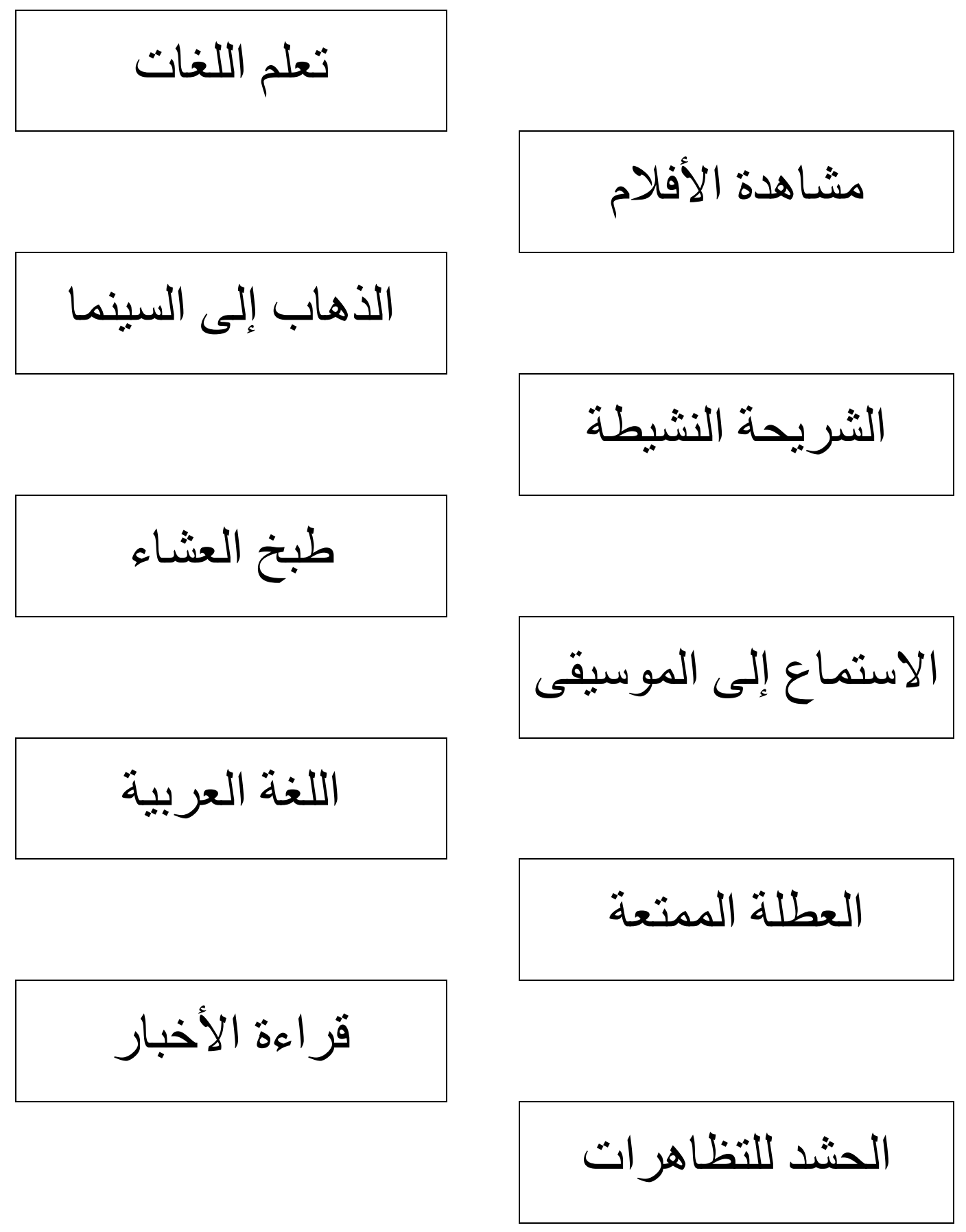


Anhang V. Vokabelkarten für die Transferphase der theoretischen Unterrichtseinheit

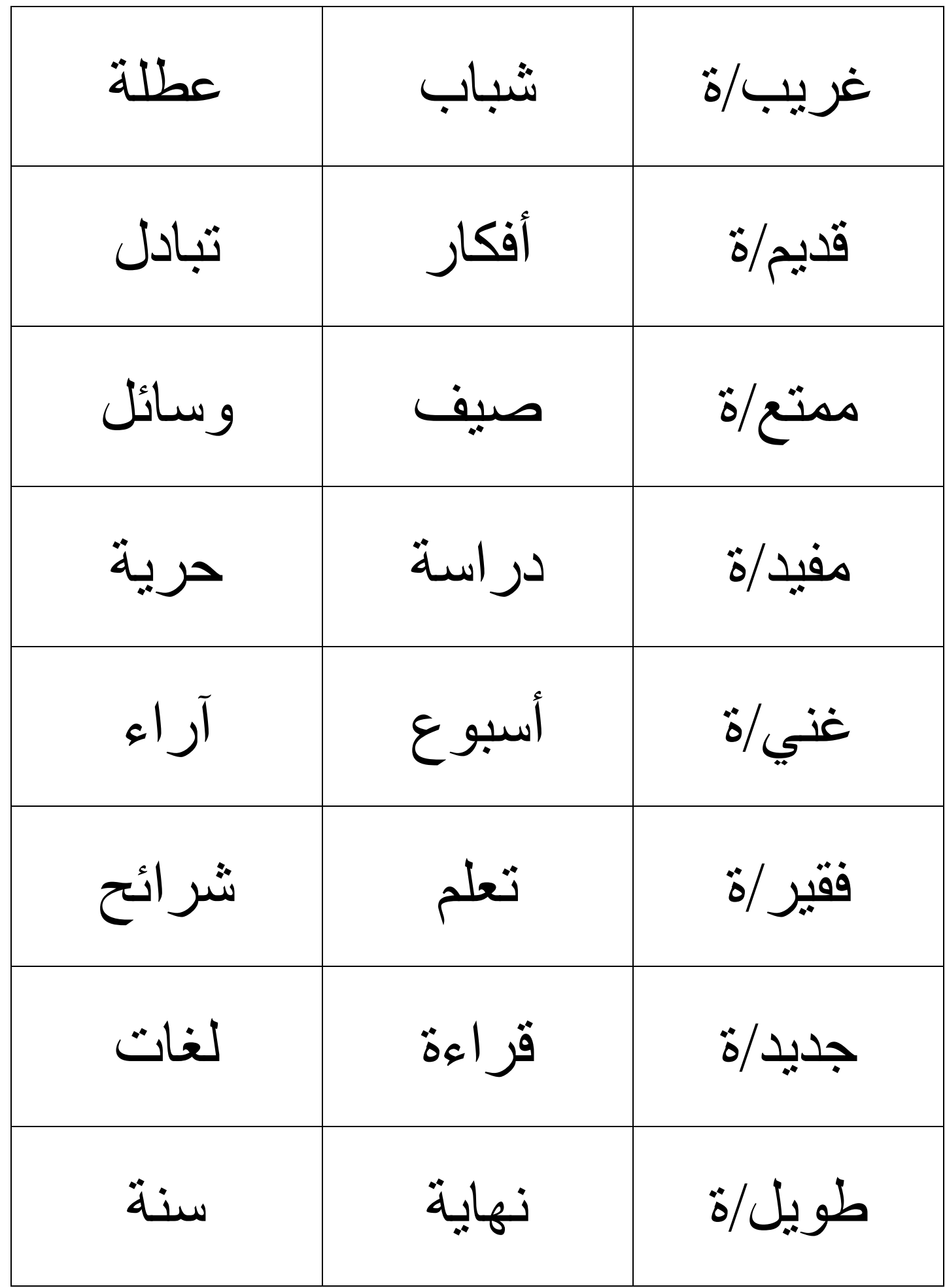


Anhang VI. Lückentext für die Transferphase der theoretischen Unterrichtseinheit

\section{املؤوا القراغات بالمفردات الجديدة}

لـقل الأفكار و الآر اء المتعلقة

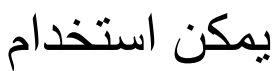
بموضوع معين لعدد كبير من الأشخاص وبطريقة سهلة، وذلك من أي مكان، وبالإضافة إلى ذللك تساهم مو اقع التو اصل الاجتماعي في وتوسيع فرص المشاركة في التعبير عن الرأي.

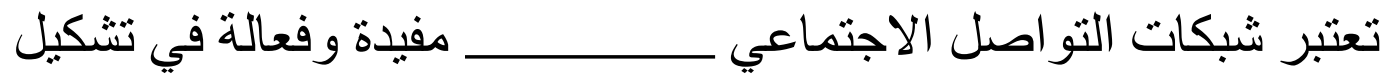
صداقات جديدة وتسهيل التواصل مع الأصدقاء البعيدين عنا، أو مع لهع الأشخاص الذين لا يمكن مقابلتهم شخصياً. يستخدم بعض الناس وسائل التو اصل الاجتماعي كأداة جيدة حيث يوجد العديد من الصفحات التي تبيع الملابس

و المكياج وحتى السيار ات بتكلفة أقل.

يمكن أن يؤدي استخدام وسائل التو اصل الاجتماعي إلى زيادة عدد

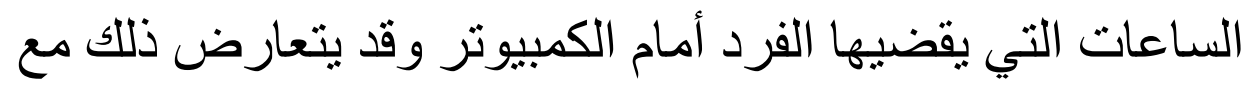
مسؤولياته اليومية ويمنعه من ممارسة حياة

تتعاون شبكات التو اصل الاجتماعي مع الثركات الكبرى في الحصول على المعلومات الخاصة من خلال متابعة نثطات الفرد على مو اقع التو اصل الاجتماعي، و حتى يمكنها الحصول على معلومات حساسة

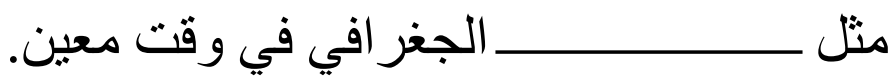




\section{Quellenverzeichnis}

Das Literaturverzeichnis ist nach Primär- und Sekundärquellen sortiert. Manche Quellen können jedoch nicht eindeutig zugeordnet werden und sind dementsprechend gekennzeichnet.

\section{Primärquellen}

Alberta Education (2018): Arabic Language Arts. Kindergarten to Grade 12. Alberta, Kanada. Online verfügbar unter https://education.alberta.ca/media/3772294/arabic-language-arts-k-12.pdf, zuletzt geprüft am 18.03.2020.

Aldrich, Matthew (2014): Arabic Voices 1. Authentic Listening and Reading Practice in Modern Standard Arabic and Colloquial Dialects: Lingualism.

Aljazeera Media Network (2020): Learning Arabic. Online verfügbar unter https://learning.aljazeera.net/en, zuletzt geprüft am 29.06.2020.

Board of Education Virginia Commonwealth (2014): Foreign Language Standards of Learning for Virginia Public Schools. Richmond, VA. Online verfügbar unter

http://www.doe.virginia.gov/testing/sol/standards_docs/foreign_language/index.s html, zuletzt geprüft am 19.03.2020.

Board of Studies New South Wales (Hg.) (2003): Arabic K-10. Syllabus. Sydney, Australien. Online verfügbar unter

https://www.boardofstudies.nsw.edu.au/syllabus_sc/pdf_doc/arabic_k10_syl.pdf, zuletzt geprüft am 19.03.2020.

Defense Language Institute Foreign Language Center: Accents Library. Online verfügbar unter https://accents.DLIFLC.edu/, zuletzt geprüft am 16.07.2020.

Defense Language Institute Foreign Language Center: FAMiliarization. Online verfügbar unter https://fieldsupport.DLIFLC.edu/productList.aspx?v=lsk, zuletzt geprüft am 16.07.2020.

DLIFLC - Defense Language Institute Foreign Language Center: eLearning. Online verfügbar unter https://www.DLIFLC.edu/elearning/, zuletzt geprüft am 16.07.2020.

DLIFLC - Defense Language Institute Foreign Language Center: GLOSS.

Online verfügbar unter https://GLOSS.DLIFLC.edu/, zuletzt geprüft am 16.07.2020. 
DLIFLC - Technology Integration (2010): Arabic Phone Conversations. Online verfügbar unter https://phone.DLIFLC.edu/default_arabic.asp, zuletzt geprüft am 16.07.2020.

Duolingo, Inc. (2020): Duolingo (Version 6.75.0) [Mobile

Anwendungssoftware]. Online verfügbar unter

https://apps.apple.com/de/app/duolingo/id570060128, zuletzt geprüft am 16.07.2020.

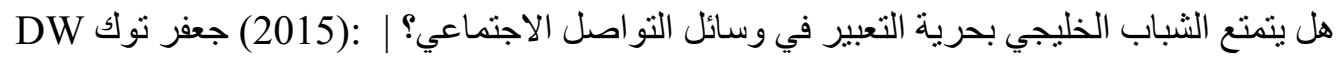
207.01.2015. [YouTube-Video]. Online verfügbar unter https://www.youtube.com/watch?v=njct3PuxDRQ, zuletzt geprüft am 25.06.2020. ${ }^{233}$

Edmonton Public Schools (2020): Arabic Bilingual. Program Description. Online verfügbar unter https://epsb.ca/programs/language/arabicbilingual/, zuletzt geprüft am 06.04.2020.

Fairfax County Public Schools (2020a): Arabic Level 1. Online verfügbar unter https://www.fcps.edu/year-at-a-glance/arabic-level-1, zuletzt geprüft am 22.03.2020.

Fairfax County Public Schools (2020b): Arabic Level 2. Online verfügbar unter https://www.fcps.edu/year-at-a-glance/arabic-level-2, zuletzt geprüft am 22.03.2020.

Fairfax County Public Schools (2020c): Arabic Level 3. Online verfügbar unter https://www.fcps.edu/year-at-a-glance/arabic-level-3, zuletzt geprüft am 22.03.2020.

Fairfax County Public Schools (2020d): Arabic Upper Level. Online verfügbar unter https://www.fcps.edu/year-at-a-glance/arabic-upper-level, zuletzt geprüft am 22.03.2020.

InterLingo Languages (2020a): InterLingo. Online verfügbar unter https://www.interlingo.co.uk/, zuletzt geprüft am 16.07.2020.

Khallina (2020a): Home. Online verfügbar unter https://khallina.org/, zuletzt geprüft am 19.06.2020.

Khallina (2020b): Women Empowerment. Online verfügbar unter https://khallina.org/module/women-empowerment/, zuletzt geprüft am 19.06.2020.

\footnotetext{
${ }^{233}$ Der Inhalt des Videos dient als Ausgangsbasis für die Präsentationsphase der theoretischen Unterrichtseinheit. Das Video selbst wird jedoch nicht ausführlich analysiert oder bewertet. Folglich könnte diese Quelle auch als Sekundärquelle betrachtet werden.
} 
Lingualism (2020a): Languages. Online verfügbar unter

https://lingualism.com/\#languages, zuletzt geprüft am 16.07.2020.

Lingualism: Lingualism Arabic. Online verfügbar unter https://www.youtube.com/user/lingualism, zuletzt geprüft am 16.07.2020.

Mawdoo3 (2020): سلبيات و إيجابيات مو اقع التو اصل الاجتماعي. Online verfügbar unter https://mawdoo3.com/سلبيات_و إيجابيات_مواقع_التواصل_الاجعتياعـ, zuletzt geprüft am 25.06.2020. ${ }^{234}$

Ministère de l'Éducation nationale (2009): Contenus Culturels et Linguistiques: Arabe. Online verfügbar unter https://langue-arabe.fr/IMG/pdf/voieprofessionnelle_programmes.pdf, zuletzt geprüft am 16.03.2020.

Ministère de l'Éducation nationale (2010): Langues vivantes - Enseignements communs, d'exploration et facultatif. Online verfügbar unter https://languearabe.fr/IMG/pdf/2de_Programmes-communs.pdf, zuletzt geprüft am 16.03.2020.

Playaling (a). Online verfügbar unter https://playaling.com/, zuletzt geprüft am 16.07.2020.

Thüringer Kultusministerium (2007): Lehrplan für die Salzmannschule Schnepfenthal Staatliches Spezialgymnasium für Sprachen Waltershausen. Erprobungsfassung für die Klassenstufen 11/12.

Thüringer Kultusministerium (2002): Lehrplan für die Salzmannschule Schnepfenthal Staatliches Spezialgymnasium für Sprachen Waltershausen. Erprobungsfassung für die Klassenstufen 6-10.

\section{Sekundärquellen}

Abdalla, Mahmoud; Al-Batal, Mahmoud (2011-2012): College-Level Teachers of Arabic in the United States. A Survey of their Professional and Institutional Profiles and Attitudes. In: Al-'Arabiyya (44/45), S. 1-28.

Al-Batal, Mahmoud (2019): Academic Writing and Communication Skills, 12.04.2019. [YouTube-Video] Online verfügbar unter https://www.youtube.com/watch?v=9V6zDCt2bRk, zuletzt geprüft am 28.04.2020.

\footnotetext{
${ }^{234}$ Der Inhalt des Artikels dient als Ausgangsbasis für die Transferphase der theoretischen Unterrichtseinheit. Der Artikel selbst wird jedoch nicht ausführlich analysiert oder bewertet. Folglich könnte diese Quelle auch als Sekundärquelle betrachtet werden.
} 
Al-Batal, Mahmoud (2018): Dialect Integration in the Arabic Foreign Language Curriculum. Vision, Rationale, and Models. In: Mahmoud Al-Batal (Hg.): Arabic as One language. Integrating Dialect in the Arabic Language Curriculum. Washington, DC: Georgetown University Press, S. 3-22.

Al-Batal, Mahmoud; Glakas, Christian (2018): Dialect Integration. Students' Perspectives within an Integrated Program. In: Mahmoud Al-Batal (Hg.): Arabic as One language. Integrating Dialect in the Arabic Language Curriculum. Washington, DC: Georgetown University Press, S. 260-278.

Aldeen Foundation; Qatar Foundation International (2013): Aldeen Teachers Professional Development. Arabic Online Education Fall 2013 VIDEOS. Online verfügbar unter https://aldeenfoundation.org/online-arabic-training/aldeenqfimaterial/, zuletzt geprüft am 27.04.2020.

Australian Trade and Investment Commission (2017): Australian Education Technology. The Australian education system. Online verfügbar unter https://www.austrade.gov.au/edtech/the-australian-education-system/, zuletzt geprüft am 08.04.2020.

Baicheng, Zhang (2009): Do example sentences work in direct vocabulary learning? In: Issues in Educational Research 19 (2), S. 175-189.

Batrawy, Aya (2017): Understanding the Al-Jazeera controversy. In: York Dispatch, 08.08.2017. Online verfügbar unter https://eu.yorkdispatch.com/story/money/business/2017/08/08/understanding-aljazeera-controversy/104408262/, zuletzt geprüft am 17.06.2020.

Behzadi, Lale; Konerding, Peter (2018): Sprache als Kulturvermittlerin - das Beispiel Arabisch. In: Adrianna Hlukhovych, Benjamin Bauer und Katharina Beuter (Hg.): Kultur und kulturelle Bildung. Interdisziplinäre Verortungen Lehrerinnen- und Lehrerbildung - Perspektiven für die Schule. Bamberg: University of Bamberg Press (Forum Lehrerinnen- und Lehrerbildung), S. 235252 .

Behzadi, Lale; Konerding, Peter; Nerowski, Christian (2019): Arabisch als moderne Fremdsprache im Schulunterricht. In: Zeitschrift für Interkulturellen Fremdsprachenunterricht 24 (2), S. 405-426.

Belnap, R. Kirk (2018): Lessons Learned and Empirical Data from Twenty-Five Years of Using an Integrated Approach. In: Mahmoud Al-Batal (Hg.): Arabic as One language. Integrating Dialect in the Arabic Language Curriculum.

Washington, DC: Georgetown University Press, S. 36-53.

Ehlion Team (2019): Weltsprachen: Welche Sprachen werden am meisten gesprochen? In: Ehlion Magazine, 19.07.2019. Online verfügbar unter https://ehlion.com/de/meistgesprochene-sprachen/. 
Brustad, Kristen (2006): Reading Fluently in Arabic. In: Kassem M. Wahba, Zeinab A. Taha und Liz England (Hg.): Handbook for Arabic Language Teaching Professionals in the $21^{\text {st }}$ Century. Mahwah, N.J.: Lawrence Erlbaum Associates, S. 341-352.

Brustad, Kristen; Al-Batal, Mahmoud; Al-Tonsi, Abbas (2015): Al-Kitaab fii Ta allum al-'Arabiyya. A Textbook for Beginning Arabic: Part One. Third edition, Updated. Washington, DC: Georgetown University Press.

Bundesverband der Dolmetscher und Übersetzer (2017): Staaten und ihre Sprachen. Amtssprachen, Gerichtssprachen, Handelssprachen, Verkehrssprachen, Bildungssprachen, Umgangssprachen. Online verfügbar unter https://bw.bdue.de/fileadmin/files/PDF/Publikationen/BDUe_Staaten_und_ihre_ Sprachen.pdf, zuletzt geprüft am 26.06.2020.

Council of Europe: Global scale - Table 1 (CEFR 3.3): Common Reference levels. Online verfügbar unter https://www.coe.int/web/common-europeanframework-reference-languages/table-1-cefr-3.3-common-reference-levelsglobal-scale, zuletzt geprüft am 26.06.2020.

DLIFLC - Defense Language Institute Foreign Language Center: About. Online verfügbar unter https://www.DLIFLC.edu/about/, zuletzt geprüft am 16.06.2020.

Duolingo (a): for schools. Online verfügbar unter https://schools.duolingo.com/, zuletzt geprüft am 15.06.2020.

Duolingo (b): Kostenloser Sprachunterricht. Für immer. Online verfügbar unter https://de.duolingo.com/, zuletzt geprüft am 15.06.2020.

Duolingo (c): Über uns. Ansatz. Online verfügbar unter https://de.duolingo.com/approach, zuletzt geprüft am 15.06.2020.

Duolingo (d): Über uns. Mission. Online verfügbar unter https://de.duolingo.com/info, zuletzt geprüft am 15.06.2020.

Eickelmann, Birgit; Schaumburg, Heike; Drossel, Kerstin; Lorenz, Ramona (2014): Schulische Nutzung von neuen Technologien in deutschen Schulen im internationalen Vergleich. In: Wilfried Bos, Birgit Eickelmann, Julia Gerick, Frank Goldhammer, Heike Schaumburg, Knut Schwippert et al. (Hg.): ICILS 2013 - Computer- und informationsbezogene Kompetenzen von Schülerinnen und Schülern in der 8. Jahrgangsstufe im internationalen Vergleich. Münster, New York: Waxmann Verlag, S. 197-229.

Fachverband Arabisch e.V. (2020): Über uns. Online verfügbar unter https://fachverband-arabisch.de/index.php?id=30, zuletzt geprüft am 04.01.2020.

Fairfax County Public Schools (2020e): Arabic. Course Information. Online verfügbar unter https://www.fcps.edu/academics/high-school-academics-912/world-languages/arabic, zuletzt geprüft am 07.04.2020. 
Featherstone, Jonathan (2018): Preparing Arabic Teachers for Integration. The Edinburgh Model. In: Mahmoud Al-Batal (Hg.): Arabic as One language. Integrating Dialect in the Arabic Language Curriculum. Washington, DC: Georgetown University Press, S. 54-72.

Friedrich-Ebert-Stiftung (2017): Konzepte und Entwicklungsperspektiven. Kompetenzen in der digitalen Welt. Online verfügbar unter https://library.fes.de/pdf-files/studienfoerderung/13644.pdf, zuletzt geprüft am 25.03.2020.

Galloway, Vicki (1998): Constructing cultural realities. "Facts" and frameworks of association. In: Jane Harper, Madeleine G. Lively und Mary Karen Williams (Hg.): The coming of age of the profession. Issues and emerging ideas for the teaching of foreign languages. Boston: Heinle \& Heinle, S. 129-140.

Georgetown University Press: Al-Kitaab Arabic Language Program. Online verfügbar unter https://alkitaabtextbook.com/, zuletzt geprüft am 29.04.2020.

Georgetown University Press: Al-Kitaab Part 1, Third Edition. Online verfügbar unter https://alkitaabtextbook.com/part1/, zuletzt geprüft am 29.04.2020.

Giolfo, Manuela E.B.; Salvaggio, Federico (2018): A Digitally Assisted Model of Integration of Standard and Colloquial Arabic Based on the Common European Framework. In: Mahmoud Al-Batal (Hg.): Arabic as One language. Integrating Dialect in the Arabic Language Curriculum. Washington, DC: Georgetown University Press, S. 93-113.

Grünewald, Andreas (2016): Digitale Medien und soziale Netzwerke im Kontext des Lernens und Lehrens von Sprachen. In: Eva Burwitz-Melzer, Grit Mehlhorn, Claudia Riemer, Karl-Richard Bausch und Hans-Jürgen Krumm (Hg.): Handbuch Fremdsprachenunterricht. 6., völlig überarbeitete und erweiterte Auflage.

Tübingen: A. Francke Verlag (UTB, 8043), S. 463-466.

Guder, Andreas (2005): „Kann man das überhaupt lernen?“. Zur Vermittlung von Chinesisch als distanter Fremdsprache. In: Lebende Sprachen 50 (2), S. 61-67.

Hildebrandt, Thomas: Die wissenschaftliche Umschrift der arabischen Sprache. Ein Leitfaden für die orientalistischen Fächer der Universität Bamberg. Online verfügbar unter https://www.unibamberg.de/fileadmin/uni/fakultaeten/split_faecher/orientalistik/Dateien/Translit. pdf, zuletzt geprüft am 21.07.2020.

Institut für Pädagogik der Elementar- und Primarstufe (2011): Einführungsreferat in die Digitalen Medien. Online verfügbar unter http://grundschule.bildung.hessen.de/ws1112/20111026_1_handout.pdf, zuletzt geprüft am 24.04.2020. 
InterLingo Languages (2020b): About. Online verfügbar unter

https://www.interlingo.co.uk/about/, zuletzt geprüft am 15.06.2020.

Isleem, Martin (2018): Integrating Colloquial Arabic in the Classroom. A Study of Students' and Teachers' Attitudes and Effects. In: Mahmoud Al-Batal (Hg.): Arabic as One language. Integrating Dialect in the Arabic Language Curriculum. Washington, DC: Georgetown University Press, S. 237-259.

Kampmann, Elisabeth; Schwering, Gregor (2017): Teaching Media. Medientheorie für die Schulpraxis - Grundlagen, Beispiele, Perspektiven. Bielefeld: transcript Verlag.

Klafki, Wolfgang (2007): Neue Studien zur Bildungstheorie und Didaktik. Zeitgemäße Allgemeinbildung und kritisch-konstruktive Didaktik. 6. Aufl. Weinheim und Basel: Beltz Verlag.

Kühn, Alexander; Reuter, Christoph; Schmitz, Gregor Peter (2013): Al-Jazeera Losing Battle for Independence. In: Spiegel International, 15.02.2013. Online verfügbar unter https://www.spiegel.de/international/world/al-jazeera-criticizedfor-lack-of-independence-after-arab-spring-a-883343.html, zuletzt geprüft am 17.06.2020.

Landesmedienzentrum Baden-Württemberg (2019): Grundbegriffe der Mediendidaktik. Online verfügbar unter https://www.lmz-bw.de/medien-undbildung/medienbildung/grundlagen-der-mediendidaktik/grundbegriffe-dermediendidaktik/, zuletzt geprüft am 23.04.2020.

Lehrerinnenfortbildung Baden-Württemberg (2020): Was sind WebQuests? Online verfügbar unter https://lehrerfortbildungbw.de/u_gestaltlehrlern/projekte/webquest/definition.html, zuletzt geprüft am 21.04.2020.

Levallois, Bruno (2009): L'enseignement de l'arabe dans l'institution scolaire française. In: Langues et cité (15), S. 6-7.

Lingualism (2020b): About. Online verfügbar unter https://lingualism.com/about/, zuletzt geprüft am 29.06.2020.

Looney, Dennis; Lusin, Natalia (2018): Enrollments in Languages Other Than English in United States Institutions of Higher Education, Summer 2016 and Fall 2016: Preliminary Report. Hg. v. Modern Language Association of America. Online verfügbar unter https://www.mla.org/Resources/Research/SurveysReports-and-Other-Documents/Teaching-Enrollments-andPrograms/Enrollments-in-Languages-Other-Than-English-in-United-StatesInstitutions-of-Higher-Education, zuletzt geprüft am 02.05.2020. 
Madhany, Al-Husein N. (2006): Teaching Arabic with Technology: Word Processing, E-Mail, and the Internet. In: Kassem M. Wahba, Zeinab A. Taha und Liz England (Hg.): Handbook for Arabic Language Teaching Professionals in the $21^{\text {st }}$ Century. Mahwah, N.J.: Lawrence Erlbaum Associates, S. 295-303.

Ministère de l'Éducation nationale, de l'Enseignement supérieur et de la Recherche (2020): Langue et Culture arabes. Online verfügbar unter https://langue-arabe.fr/, zuletzt geprüft am 04.03.2020.

Najour, Caroline (2018): Teacher's Voices. Analysis of Teachers' Speech and Teachers' Perspectives in Integrated Arabic Classrooms. In: Mahmoud Al-Batal (Hg.): Arabic as One language. Integrating Dialect in the Arabic Language Curriculum. Washington, DC: Georgetown University Press, S. 298-317.

Neuß, Norbert (2005): Medienbildung als eigenständiges Lern- und Themenfeld. In: Medienimpulse (51), S. 59-63.

Nieweler, Andreas (Hg.) (2017): Fachdidaktik Französisch. Das Handbuch für Theorie und Praxis. 1. Auflage. Stuttgart: Ernst Klett Sprachen GmbH.

Nikolova, Ofelia R. (2002): Effects of Students' Participation in Authoring of Multimedia Materials on Student Acquisition of Vocabulary. In: Language Learning \& Technology 6 (1), S. 100-122.

Otto-Friedrich-Universität Bamberg, Arabistik (2019): Aktivitäten. Online verfügbar unter https://www.uni-bamberg.de/arabistik/aktivitaeten/, zuletzt geprüft am 30.03.2020.

Plassmann, Senay (1992): Grammatik im Fremdsprachenunterricht. In: Hacettepe University Journal of Education (7), S. 339-349.

Playaling (b): About Playaling. Online verfügbar unter https://playaling.com/about, zuletzt geprüft am 17.06.2020.

Prensky, Marc (2001): Digital Natives, Digital Immigrants. In: On the Horizon 9 (5), S. 1-6.

Qatar Foundation International (2020): Concordia Language Villages - QFI Arabic Teacher Professional Development Workshops | Qatar Foundation International. Online verfügbar unter https://www.qfi.org/opportunities/concordia-language-villages-qfi-arabicteacher-professional-development-workshops/, zuletzt geprüft am 27.04.2020.

Ramadan, Dunja (2017): Al Jazeera - gefürchtete Stimme der Massen, 25.06.2017. Online verfügbar unter https://www.sueddeutsche.de/medien/imvisier-der-nachbarn-al-jazeera-gefuerchtete-stimme-der-massen-1.3558089, zuletzt geprüft am 17.06.2020. 
Ryding, Karin C. (2015): Arabic Language Learning. If Diglossia is the Question, then What is the Answer? In: Annals of Japan Association for Middle East Studies 31 (2), S. 63-82.

Salzmannschule Schnepfenthal (2020): Fremdsprachen. Online verfügbar unter http://www.salzmannschule.de/index.php/home/fremdsprachen, zuletzt geprüft am 01.04.2020.

Samy, Waheed (2006): Instructional Media and Learning Arabic. In: Kassem M. Wahba, Zeinab A. Taha und Liz England (Hg.): Handbook for Arabic Language Teaching Professionals in the $21^{\text {st }}$ Century. Mahwah, N.J.: Lawrence Erlbaum Associates, S. 263-273.

Sasse, Sabine (2015): Stimme der Jugend. In: Der Tagesspiegel, 09.09.2015. Online verfügbar unter https://www.tagesspiegel.de/gesellschaft/medien/shababtalk-stimme-der-jugend/12298830.html, zuletzt geprüft am 25.06.2020.

Sekretariat der Kultusministerkonferenz (Hg.) (2016): Bildung in der digitalen Welt. Strategie der Kultusministerkonferenz. Online verfügbar unter https://www.kmk.org/fileadmin/Dateien/veroeffentlichungen_beschluesse/2018/S trategie_Bildung_in_der_digitalen_Welt_idF._vom_07.12.2017.pdf, zuletzt geprüft am 30.04.2020.

Sekretariat der Ständigen Konferenz der Kultusminister (Hg.) (2012):

Bildungsstandards für die fortgeführte Fremdsprache (Englisch/Französisch) für die Allgemeine Hochschulreife. (Beschluss der Kultusministerkonferenz vom 18.10.2012). Online verfügbar unter https://www.kmk.org/fileadmin/Dateien/veroeffentlichungen_beschluesse/2012/2 012_10_18-Bildungsstandards-Fortgef-FS-Abi.pdf, zuletzt geprüft am 30.04.2020.

Shiri, Sonia; Joukhadar, Charles (2018): Arabic Diglossic Speaking without Mixing. In: Mahmoud Al-Batal (Hg.): Arabic as One language. Integrating Dialect in the Arabic Language Curriculum. Washington, DC: Georgetown University Press, S. 154-172.

Sommer, Fabian (2020): Maas will Goethe-Institut vor Einschnitten wegen Corona bewahren. In: Der Tagesspiegel, 10.04.2020. Online verfügbar unter https://www.tagesspiegel.de/kultur/kulturpolitik-im-ausland-maas-will-goetheinstitut-vor-einschnitten-wegen-corona-bewahren/25732330.html, zuletzt geprüft am 15.07.2020.

Snowden, Emma; Soliman, Rasha, Towler, Melissa (2016): Teaching Arabic as a Foreign Language in the UK. Strand 1 Research: How Arabic is being taught in schools. British Council; Goldsmiths University of London, University of Leeds. Online verfügbar unter https://www.britishcouncil.org/sites/default/files/research_into_teaching_arabic_ as_a_foreign_language_in_the_uk.pdf, zuletzt geprüft am 05.04.2020. 
Spanhel, Dieter (2011): Medienbildung im Spannungsfeld medienpädagogischer Leitbegriffe. In: MedienPädagogik: Zeitschrift für Theorie und Praxis der Medienbildung 20, S. 95-120.

Ständige Konferenz der Kultusminister der Länder in der Bundesrepublik Deutschland (2011): Empfehlungen der Kultusministerkonferenz zur Stärkung der Fremdsprachenkompetenz. (Beschluss der Kultusministerkonferenz vom 08.12.2011). Online verfügbar unter https://www.kmk.org/fileadmin/Dateien/veroeffentlichungen_beschluesse/2011/2 011_12_08-Fremdsprachenkompetenz.pdf, zuletzt geprüft am 21.03.2020.

Ständige Konferenz der Kultusminister der Länder in der Bundesrepublik Deutschland (2012): Medienbildung in der Schule. (Beschluss der Kultusministerkonferenz vom 8. März 2012). Online verfügbar unter https://www.kmk.org/fileadmin/Dateien/veroeffentlichungen_beschluesse/2012/2 012_03_08_Medienbildung.pdf, zuletzt geprüft am 22.03.2020.

Ständige Konferenz der Kultusminister der Länder in der Bundesrepublik Deutschland (2016): Kompetenzen in der digitalen Welt. Kompetenzbereiche. Online verfügbar unter https://www.kmk.org/fileadmin/Dateien/pdf/PresseUndAktuelles/2017/KMK_Ko mpetenzen_-_Bildung_in_der_digitalen_Welt_Web.html, zuletzt geprüft am 25.03.2020.

Stevens, Vance (2006): Learner Strategies at the Interface: Computer-Assisted Language Learning Meets Computer-Mediated Communication. In: Kassem M. Wahba, Zeinab A. Taha und Liz England (Hg.): Handbook for Arabic Language Teaching Professionals in the $21^{\text {st }}$ Century. Mahwah, N.J.: Lawrence Erlbaum Associates, S. 253-262.

Technische Universität Dresden (2017): Handreichung zu ersten Unterrichtsplanungen. Online verfügbar unter https://tudresden.de/gsw/phil/ige/nngdg/ressourcen/dateien/materialien-blockpraktikumb/10-Handreichung-zu-ersten-Unterrichtsplanungen.pdf?lang=en, zuletzt geprüft am 25.06.2020.

Trentman, Emma (2018): Developing a Genre-Based Curriculum to Teach Arabic Diglossia. In: Mahmoud Al-Batal (Hg.): Arabic as One language. Integrating Dialect in the Arabic Language Curriculum. Washington, DC: Georgetown University Press, S. 114-133.

Turner, Mike (2018): An Integrated Moroccan and Modern Standard Arabic Curriculum for First-Year Learners. In: Mahmoud Al-Batal (Hg.): Arabic as One language. Integrating Dialect in the Arabic Language Curriculum. Washington, DC: Georgetown University Press, S. 134-153. 
United Nations: Official Languages. Online verfügbar unter https://www.un.org/en/sections/about-un/official-languages/index.html, zuletzt geprüft am 26.06.2020.

Van Mol, Mark (2006): Arabic Receptive Language Teaching: A New CALL Approach. In: Kassem M. Wahba, Zeinab A. Taha und Liz England (Hg.): Handbook for Arabic Language Teaching Professionals in the $21^{\text {st }}$ Century. Mahwah, N.J.: Lawrence Erlbaum Associates, S. 305-316.

Verbraucherzentrale Bundesverband: Bewertungskriterien. Kriterien zur Bewertung von Unterrichtsmaterialien in der Verbraucherbildung. Online verfügbar unter

https://www.verbraucherbildung.de/materialkompass/bewertungskriterien, zuletzt geprüft am 10.06.2020.

Verbraucherzentrale Bundesverband (2016): Digitale Bildung Gestalten. Stellungnahme des Verbraucherzentrale Bundesverbands zur Strategie der Kultusministerkonferenz „Bildung in der digitalen Welt“. Online verfügbar unter https://www.vzbv.de/sites/default/files/2016-12-

16_stellungnahme_vzbv_kmk_strategie_dig_bildung.pdf, zuletzt geprüft am 29.06.2020.

Vollstädt, Witlof; Tillmann, Klaus-Jürgen; Rauin, Udo; Höhmann, Katrin; Tebrügge, Andrea (1999): Lehrpläne im Schulalltag. Eine empirische Studie zur Akzeptanz und Wirkung von Lehrplänen in der Sekundarstufe I. Opladen: Leske + Budrich.

Vormbaum, Ulrich: Wolfgang Klafkis Begriff der Bedeutung zur Begründung von Unterrichtsinhalten. Online verfügbar unter https://www.vormbaum.net/index.php/latest-downloads/universitaet-konstanzstudenten/downloads-fuer-fachdidaktik-deutsch-i/hinweise-fuer-die-hausarbeitin-fd-1/2200-klafki-der-begriff-der-bedeutung/file, zuletzt geprüft am 25.06.2020.

Wei, Chun-Wang; Kao, Hao-Yun; Lu, Hsin-Hsien; Liu, Yi Chun (2018): The Effects of Competitive Gaming Scenarios and Personalized Assistance Strategies on English Vocabulary Learning. In: Journal of Educational Technology \& Society 21 (3), S. 146-158.

Zaki, Mai; Palmer, Jeremy (2018): Integration and Students' Perspectives in a Multidialect Environment. In: Mahmoud Al-Batal (Hg.): Arabic as One language. Integrating Dialect in the Arabic Language Curriculum. Washington, DC:

Georgetown University Press, S. 279-297.

Zimmermann, Günther (1988): Lehrphasenmodell für den fremdsprachlichen Grammatikunterricht. In: Johannes Dahl und Brigitte Weis (Hg.): Grammatik im Unterricht. Expertisen und Gutachten zum Projekt „Grammatik im Unterricht“ des Goethe-Instituts München. München: Goethe-Institut, S. 160-177. 